\title{
Characterization of the CNS-specific F-box protein FBXO41 in cerebellar development
}

\author{
Dissertation \\ for the award of the degree \\ "Doctor rerum naturalium" \\ of the Georg-August-Universität Göttingen \\ within the doctoral program \\ Molecular Physiology of the Brain \\ of the Georg-August-University School of Science (GAUSS)
}

submitted by

\section{Anna Holubowska}

from Walcz, Poland

Göttingen 2013 


\section{Members of the thesis committee}

Dr. Judith Stegmüller Laboratory of Cellular and Molecular Neurobiology, Max Planck Institute of experimental Medicine, Göttingen

Prof. Dr. Dr. Hannelore Ehren reich Division of Clinical Neuroscience, Max Planck Institute of Experimental Medicine, Göttingen

Prof. Dr. André Fischer Laboratory for Aging and Cognitive Diseases, European Neuroscience Institute, Göttingen

\section{Members of the extended thesis committee}

Reviewer 1 Dr. Judith Stegmüller, Laboratory of Cellular and Molecular Neurobiology, Max Planck Institute of Experimental Medicine, Göttingen

Reviewer 2 Prof. Dr. Dr. Hannelore Ehrenreich, Division of Clinical Neuroscience, Max Planck Institute of Experimental Medicine, Göttingen

Prof. Dr. André Fischer, Laboratory for Aging and Cognitive Diseases, European Neuroscience Institute, Göttingen

Prof. Dr. Anastassia Stoykova, Department of Molecular Cell Biology, Max Planck Institute for Biophysical Chemistry, Göttingen

Prof. Dr. Andreas Wodarz, Stem Cell Biology, Department of Anatomy and Cell Biology, Göttingen Center of Molecular Biosciences, Göttingen

Prof. Dr. Ralf Heinrich, Department of Cellular Neurobiology, Schwann-Schleiden Research Center, Göttingen

Date of submission of the dissertation 31st August 2013

Date of the oral examination 23rd October 2013

ii 
I hereby confirm that this dissertation,

"Characterization of the CNS-specific F-box protein FBXO41 in cerebellar development”,

was prepared on my own and with no other sources or aids than quoted.

Göttingen, August 2013

Anna Holubowska 



\section{Contents}

\begin{tabular}{ll}
\hline Acknowledgments & ix
\end{tabular}

Summary $\quad$ xi

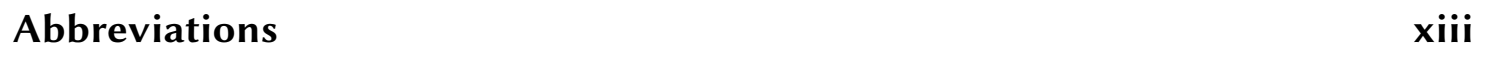

1. Introduction 1

1.1. Mechanisms of neuronal migration and morphogenesis . . . . . . . . 1

1.1.1. Neuronal migration in the telencephalon and cerebellum . . . . 2

1.1.2. Extracellular cues in neuronal migration . . . . . . . . . . 4

1.1.3. Cytoskeleton in migrating neurons . . . . . . . . . . . . 6

1.1.3.1. The Centrosome during neuronal development. . . . 7

1.1.4. DISC1 - a converging point of the signaling pathways in neuronal migration . . . . . . . . . . . . . 8

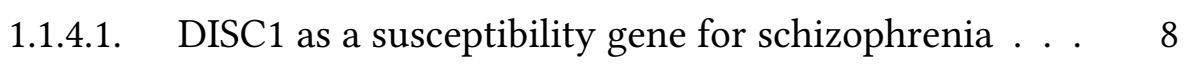

1.1.4.2. Localization of DISC1 . . . . . . . . . . . . 9

1.1.4.3. Genomic and protein structure of DISC1 . . . . . . 10

1.1.4.4. DISC1 in regulation of neuronal migration . . . . . . 11

1.1.5. Interaction of NDEL1 and DISC1 in developing brain . . . . . . 13

1.1.6. Axon specification and extension . . . . . . . . . . . . 14

1.1.7. Regulation of neuronal morphogenesis . . . . . . . . . . . . 15

1.1.8. DISC1 in neuronal morphogenesis . . . . . . . . . . . . . 17

1.2. Ubiquitin-proteasome system in neural development . . . . . . . . . . 19

1.2.1. Protein ubiquitination as a posttranslational modification . . . 20 


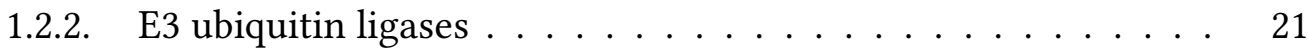

1.2.3. SCF complex and F-box proteins . . . . . . . . . . . 22

$\begin{array}{lll}\text { 1.2.3.1. F-box proteins in neuronal development and disease } 24 & 24\end{array}$

1.2.4. Aim of the project . . . . . . . . . . . . . 26

2. Materials and methods 29

2.1. Chemicals and kits . . . . . . . . . . . . . . . 29

2.2. Antibodies ......................... 29

2.3. Lab equipment . . . . . . . . . . . . . . . . . . 31

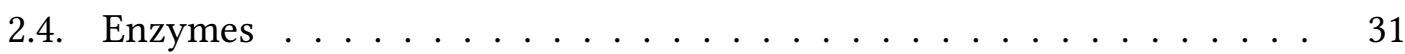

2.5. Solutions . . . . . . . . . . . . . . . . 31

2.6. Expression constructs and primers . . . . . . . . . . . . . . 33

2.7. Molecular biology methods . . . . . . . . . . . . . . . 37

2.7.1. General molecular cloning . . . . . . . . . . . . . . . . 37

2.7.2. Generation of vector-based shRNA . . . . . . . . . . . 38

2.7.3. Site-directed mutagenesis . . . . . . . . . . . . . . . 39

2.7.4. Generation of deletion mutants $(\Delta \mathrm{CTR}$ and $\Delta \mathrm{F}-\mathrm{box}) \ldots . . .40$

2.8. Cell culture . . . . . . . . . . . . . . . . 41

2.8.1. Culture of HEK 293T cells . . . . . . . . . . . . . . . . . . . 41

2.8.2. Culture of primary cerebellar granule neurons . . . . . . . . . . 41

2.8.3. Culture of primary hippocampal neurons . . . . . . . . 42

2.9. Calcium phosphate transfection . . . . . . . . . . . . . . . 43

2.9.1. Transfection of HEK 293T cells . . . . . . . . . . . . . . 43

2.9.2. Transfection of primary neurons . . . . . . . . . . . . 43

2.10. Immunocytochemistry . . . . . . . . . . . . . . 43

2.11. Morphometrical analysis . . . . . . . . . . . . . . . . . 44

2.12. Biochemical methods . . . . . . . . . . . . . . . . . . 44

2.12.1. Tissue lysate preparation . . . . . . . . . . . . 44

2.12.2. Preparation of cell lysates . . . . . . . . . . . . 45

2.12.3. Bradford assay . . . . . . . . . . . . . . . . . 45

2.12.4. SDS-PAGE and western blot . . . . . . . . . . . . . 45

vi 
2.12.5. Co-Immunoprecipitation (CoIP) . . . . . . . . . . . . . 46

2.12.6. Subcellular fractionation . . . . . . . . . . . . . . 47

2.12.7. Centrosomal purification . . . . . . . . . . . . . 47

2.13. In vivo electroporation, immunohistochemistry and data analysis . . . 47

2.14. Generation of an FBXO41 knockout mouse . . . . . . . . . . . . 48

2.15. Generation of the FBXO41 antibody . . . . . . . . . . . . . . 50

3. Results 51

3.1. Characterization of FBXO41 expression . . . . . . . . . . . . . 51

3.1.1. FBXO41 is a CNS-specific neuronal protein . . . . . . . . . 51

3.1.2. $\quad$ FBXO41 is expressed in embryonic and adult brain tissues . . . 53

3.1.3. $\quad$ FBXO41 is expressed in cultured neurons $\ldots \ldots$. . . . . . . 53

$\begin{array}{lll}\text { 3.1.4. } & \text { FBXO41 is localized to the centrosome and cytoplasm of neurons } 54 \\ 5\end{array}$

3.2. Functional analysis of FBXO41 in the neural development . . . . . . . . 55

3.2.1. The role of FBXO41 in neuronal morphogenesis . . . . . . . . 56

3.2.1.1. Knockdown of FBXO41 in CGNs inhibits axon growth 56

3.2.1.2. Axon growth is reduced in CGNs of FBXO41 knockout mice . . . . . . . . . . . . . 59

3.2.1.3. Overexpression of FBXO41 promotes axon growth in CGNs . . . . . . . . . . . . . 62

3.2.1.4. FBXO41 controls axon growth independently of the neuronal subtype . . . . . . . . . . 62

3.2.1.5. FBXO41-deficient neurons display proper dendrite growth and axon-dendrite polarity . . . . . . . . . . 63

3.2.2. FBXO41 promotes neuronal migration . . . . . . . . . . . 65

3.2.3. Survival of P5 and P16 neurons is not affected by the loss of FBXO41 ............................ 67

3.2.4. Amino acids 179-354 direct FBXO41 to the centrosome . . . . . 69

3.2.5. FBXO41 regulates axon growth in a centrosome-independent manner ............................. 71

3.2.6. Centrosomal FBXO41 promotes neuronal migration . . . . . . . 72 
3.3. Molecular mechanism of FBXO41-mediated axon growth and neuronal migration . . . . . . . . . . . . . . . . . 74

3.3.1. FBXO41 does not form a canonical Cullin1-based SCF E3 ubiquitin ligase . . . . . . . . . . . . . . . . . . . . 74

3.3.2. $\quad$ FBXO41 is a novel interacting partner of DISC1 . . . . . . . 75

3.3.3. DISC1 mediates the interaction between FBXO41 and NDEL1 . 76

3.3.4. DISC1 is not a proteolytic target of FBXO41-Cullin7 SCF complex 77

3.3.5. NDEL1 accumulates upon FBXO41 knockout . . . . . . . . . 79

3.3.6. The CTR domain of FBXO41 mediates the interaction with DISC1 79

3.3.7. FBXO41 and DISC1 act in parallel pathways to control axon growth ........................ 80

3.3.8. DISC1-binding is essential for FBXO41-mediated control of neuronal migration . . . . . . . . . . . . . . . . . 81

$\begin{array}{ll}\text { 4. Discussion } & 85\end{array}$

4.1. FBXO41 is localized to the centrosome and the cytoplasm of CNS neurons 86

4.2. $\quad$ FBXO41 promotes neuronal migration in the cerebellar cortex . . . . . 87

4.3. FBXO41 forms a Cullin7-based E3 ubiquitin ligase . . . . . . . . . . . 92

4.4. FBXO41 is a selective regulator of axon growth . . . . . . . . . . . . . 94

4.5. Perspective: FBXO41 in disease and regeneration . . . . . . . . . . 96

4.6. Conclusion . . . . . . . . . . . . . . . . . . 98

\begin{tabular}{ll}
\hline 5. Bibliography & 101
\end{tabular}

\begin{tabular}{|l|l|}
\hline A. Curriculum vitae & 141
\end{tabular} 


\section{Acknowledgments}

First of all, I would like to thank my supervisor Dr. Judith Stegmüller for her exceptional dedication in guiding me through my $\mathrm{PhD}$. I am deeply grateful to her for the excellent training and creating a working environment which makes it possible to develop as a scientist and discover own strengths. Work in Judith's lab was a great experience.

I thank the members of my thesis committee, Prof. Hannelore Ehrenreich and Prof. André Fischer, for taking the time to evaluate my project during regular meetings and for their valuable suggestions.

Next, my thanks go to all the lab members. Especially, I would like to thank Chaitali Mukherjee, with whom I shared the project, for being such a great team mate and friend. I truly enjoyed our scientific discussions, which always resulted in new ideas and inspirations. I am also grateful for all the help I got from her, both in the lab and outside the work. Many thanks to Nicola Schwedhelm-Domeyer, Dr. Madhuvanti Kannan Dr. and Mayur Vadhvani, who contributed a lot to the project. Additionally, I wish to thank David Brockelt, Shih-Ju Lee, Dr. Annika Matz and Siv Vingill for creating a great atmosphere in the lab and sharing many fantastic moments. I also thank Chaitali, Annika and David for proofreading my thesis.

I want to express my special thanks to Julian. I would not have made it without his constant support and encouragement. I am really grateful to him for being there for me in every situation.

Finally, I want to thank my parents for their faith in me and unconditional support. There are no words that could express my gratitude. 



\section{Summary}

During neurodevelopment, processes like neurogenesis, neuronal migration and morphogenesis, as well as synaptogenesis determine the shape and function of the brain. All developmental events are highly coordinated by extrinsic and intrinsic signaling pathways. The ubiquitin-proteasome system emerges as specific intrinsic regulator of brain development as well as target for therapeutic approaches. In this study, I identified the UPS component and an F-box protein FBXO41 as a CNS-specific protein expressed exclusively in neurons. I found that FBXO41 specifically stimulates axon growth in cerebellar granule neurons in vitro and in vivo. In addition, I discovered that FBXO41 is crucial for the migration of CGNs in the developing cerebellar cortex. At the molecular level, I also found that FBXO41's centrosomal localization is important for its role in neuronal migration but not for axon growth. In addition, I uncovered that FBXO41 associates with DISC1 to cooperate in neuronal migration but this interaction is dispensable for axon growth regulation. Taken together, my study led to the identification of the novel CNS-specific F-box protein FBXO41 and the characterization of its role in crucial aspects of cerebellar development. 



\section{Abbreviations}

$\begin{array}{ll}\text { AA } & \text { amino acid } \\ \text { ANOVA } & \text { analysis of variance } \\ \text { APC } & \text { anaphase promoting complex } \\ \text { APS } & \text { ammonium peroxydisulphate } \\ \text { ATP } & \text { adenosine 5'-triphosphate } \\ \text { BBS } & \text { Bardet-Biedl syndrome } \\ \text { BME } & \text { basal medium eagle } \\ \text { BSA } & \text { bovine serum albumin } \\ \text { CAMDI } & \text { coiled-coil protein associated with myosin II and DISC1 } \\ \text { CAMP } & \text { 3'-5' cyclic adenosine monophosphate } \\ \text { CC3 } & \text { cleaved caspase 3 } \\ \text { Cdc42 } & \text { cell division cycle protein 42 } \\ \text { Cdh1 } & \text { cell division cycle 20 homolog 1 } \\ \text { Cdk5 } & \text { cyclin-dependent kinase 5 } \\ \text { complementary deoxyribonucleic acid }\end{array}$




$\begin{array}{ll}\text { CGN } & \text { cerebellar granule neuron } \\ \text { CNS } & \text { central nervous system } \\ \text { CoIP } & \text { co-immunoprecipitation } \\ \text { CTR } & \text { centrosome targeting region } \\ \text { DCX } & \text { Doublecortin } \\ \text { DISC1 } & \text { disrupted in schizophrenia 1 } \\ \text { DIV } & \text { day in vitro }\end{array}$

DMEM Dulbecco's modified Eagle’s medium

DMSO dimethyl sulfoxide

DNA deoxyribonucleic acid

dNTP deoxyribonucleotide

DTT dithiothreitol

E embryonic day

EDTA ethylenediaminetetraacetic acid

EGFP enhanced GFP

EGL external granule layer

ES cells embryonic stem cells

ERK extracellular regulated kinase

GAP guanosine triphosphate hydrolase activating protein

GAPDH glyceraldehyde 3-phosphate dehydrogenase 


\begin{tabular}{|c|c|}
\hline GDP & guanosine 5'-diphosphate \\
\hline GFAP & glial fibrillary acidic protein \\
\hline GFP & green fluorescent protein \\
\hline GSK3 & glycogen synthase kinase 3 \\
\hline GTP & guanosine 5'-triphosphate \\
\hline GTPase & guanosine 5'-triphosphate hydrolase \\
\hline HECT & homologous to E6-AP C-terminus \\
\hline HEK & human embryonic kidney \\
\hline HS & horse serum \\
\hline IB & immunoblotting \\
\hline ICC & immunocytochemistry \\
\hline Id2 & inhibitor of DNA binding 2 \\
\hline IGL & internal granule layer \\
\hline IHC & immunohistochemistry \\
\hline IP & immunoprecipitation \\
\hline $\mathbf{k b}$ & kilobase \\
\hline kDa & kilodalton \\
\hline KO & knockout \\
\hline LIS1 & lissencephaly 1 \\
\hline$M$ & molar \\
\hline
\end{tabular}


MAP microtubule-associated protein

MAPK mitogen activated protein kinase

ML molecular layer

mRNA messenger ribonucleic acid

NDEL1 nuclear distribution protein nudE-like 1

NF nuclear fraction

NFM neurofilament, medium polypeptide

n.s. not significant

$\mathbf{P} \quad$ postnatal day

PAGE polyacrylamide gel electrophoresis

PAR partitioning defective

PBS phosphate buffered saline

PCM1 pericentriolar material 1

PCR polymerase chain reaction

PFA paraformaldehyde

PI3K phosphotidylinositol 3-kinase

PKC protein kinase C

PLP proteolipid protein

Rac1 ras-related C3 botulinum toxin substrate 1

Res rescue

xvi 


\begin{tabular}{|c|c|}
\hline RhoA & ras homology A \\
\hline RING & really interesting new gene \\
\hline RNA & ribonucleic acid \\
\hline RNAi & ribonucleic acid interference \\
\hline ROCK & rho kinase \\
\hline rpm & rotations per minute \\
\hline RT & room temperature \\
\hline RT-PCR & reverse transcriptase-polymerase chain reaction \\
\hline SCF complex & Skp, Cullin, F-box containing complex \\
\hline SDM & site directed mutagenesis \\
\hline SDS & sodium dodecyl sulphate \\
\hline SEM & standard error of the mean \\
\hline Skp1 & S-phase kinase-associated protein 1 \\
\hline Smurf1/2 & smad ubiquitination regulatory factor $1 / 2$ \\
\hline SNP & single nucleotide polymorphism \\
\hline TEMED & tetramethylethylenediamine \\
\hline $\mathbf{U}$ & unit \\
\hline UPS & ubiquitin-proteasome system \\
\hline UTR & untranslated region \\
\hline $\mathbf{W}$ & week \\
\hline
\end{tabular}


WNT wingless-type MMTV integration site family member

WB western blot

WT wild type

xviii 
Nucleotides

\section{1-letter code}

Adenine

A

Guanine

G

Cytosine

C

Thymine

$\mathrm{T}$

Amino acids

\section{3-letter code 1-letter code}

$\begin{array}{lll}\text { Alanine } & \text { Ala } & \text { A } \\ \text { Arginine } & \text { Arg } & \text { R } \\ \text { Asparagine } & \text { Asn } & \text { N } \\ \text { Aspartic acid } & \text { Asp } & \text { D } \\ \text { Cysteine } & \text { Cys } & \text { C } \\ \text { Glutamic acid } & \text { Glu } & \text { E } \\ \text { Glutamine } & \text { Gln } & \text { Q } \\ \text { Glycine } & \text { Gly } & \text { G } \\ \text { Histidine } & \text { His } & \text { H } \\ \text { Isoleucine } & \text { Ile } & \text { I } \\ \text { Leucine } & \text { Leu } & \text { L } \\ \text { Lysine } & \text { Lys } & \text { K } \\ \text { Methionine } & \text { Met } & \text { M } \\ \text { Phenylalanine } & \text { Phe } & \text { F } \\ \text { Proline } & \text { Pro } & \text { P } \\ \text { Serine } & \text { Ser } & \text { S } \\ \text { Threonine } & \text { Thr } & \text { T } \\ \text { Tryptophan } & \text { Trp } & \text { W } \\ \text { Tyrosine } & \text { Tyr } & \text { Y } \\ \text { Valine } & \text { Val } & \text { V } \\ \text { Unspecified } & & \text { X }\end{array}$





\section{Introduction}

\subsection{Mechanisms of neuronal migration and morphogenesis}

The mammalian brain is one of the most complex organs. Its development involves a sequence of highly orchestrated events, including neurogenesis, migration, neuronal morphogenesis and migration as well as synapse formation to integrate neurons into a fully functional network.

Neuronal morphogenesis and migration are tightly controlled by a variety of extrinsic and intrinsic mechanisms. The extracellular regulators of neural development include growth factors, extracellular matrix proteins and cell adhesion molecules (Dijkhuizen and Ghosh, 2005, Huber et al., 2003, Katz and Shatz, 1996, Markus et al., 2002a, TessierLavigne and Goodman, 1996). These factors bind extracellular receptors and trigger diverse signaling pathways. Morphological analyses of dissociated neurons in culture demonstrated however, that neurodevelopmental processes are also governed by cellintrinsic mechanisms. In particular transcription factors and the ubiquitin-proteasome system were shown to play a critical role in neural development (Konishi et al., 2004, Stegmüller et al., 2006, Jan and Jan, 2003, Arlotta et al., 2005, Arnaud et al., 2003a, Feng et al., 2007, Kim et al., 2005, Wang et al., 2003, Gaudillière et al., 2004). Ultimately the extrinsic and intrinsic pathways converge and act on the cytoskeleton (Barnes and Polleux, 2009, Chhabra and Higgs, 2007, Dehmelt and Halpain, 2004). 


\subsubsection{Neuronal migration in the telencephalon and cerebellum}

Newly born neurons migrate considerable distances to reach their destination where they form synaptic connections. Three major types of migration can be distinguished in the developing telencephalon: radial, tangential and chain migration.

During radial migration in the developing cortex, newly generated excitatory neurons migrate outwards from the subventricular zone to the pial surface. Here, laterborn neurons migrate past the ones that have already settled. As a consequence, the older neurons reside in the deeper cortical layers, while the younger neurons form the superficial layers. As these neurons migrate, they move along radial glial fibers, that extend from the subventricular zone to the pial surface. Because of this fact, radial migration is considered to be a gliophilic migration (Figure 1.1, Rakic (1978)).

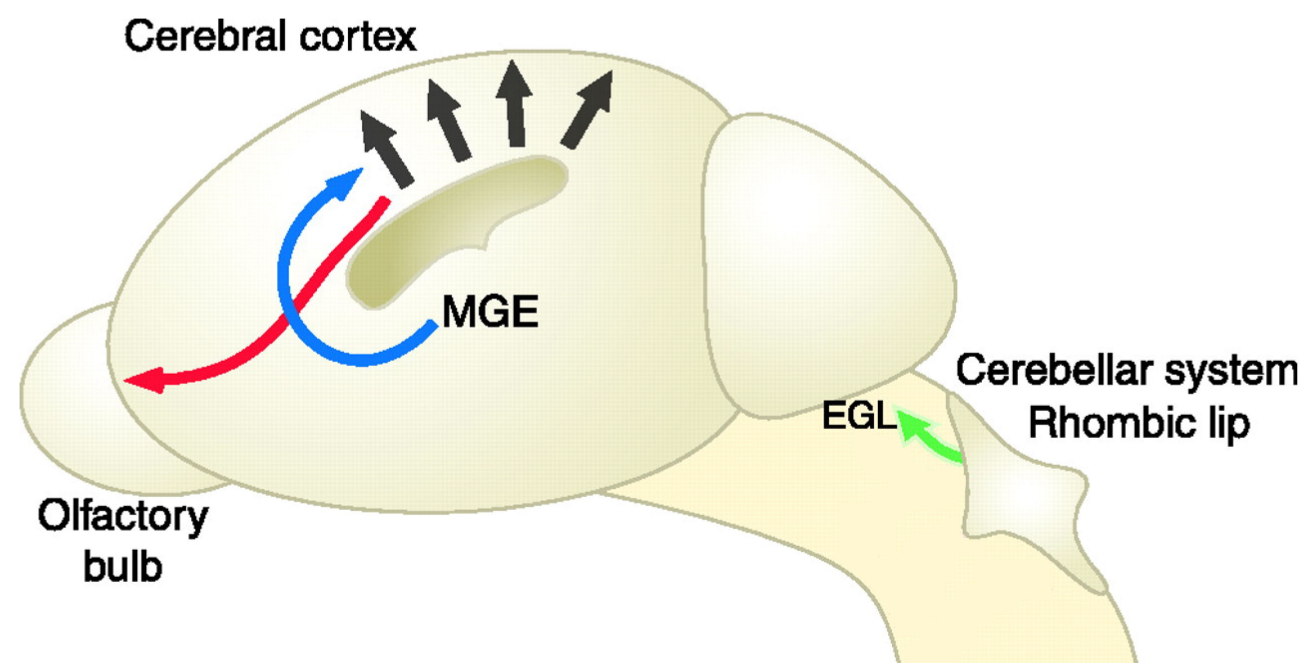

Figure 1.1.: Routes of neuronal migration in the developing telencephalon and the cerebellum. Inhibitory interneuron progenitors originate from medial ganglionic eminence (MGE) and migrate tangentially towards the cortical plate (blue arrow). Excitatory projection neurons are generated from progenitors residing in the subventricular zone and migrate radially towards the pial surface in a glia-guided manner (black arrows). Progenitors of the cerebellar granule neurons migrate tangentially from the upper rhombic lip to the external granule layer (EGL) where they generate neurons (green arrow). These in turn, migrate radially to reach the internal granule layer. Modified from Hatten (2002).

2 
In contrast to radial migration, which applies mostly to excitatory neurons, GABAergic interneurons that originate from the lateral and medial ganglionic eminences migrate tangentially to reach the cortex. Another difference between the tangential and radial migration is the developmental stage of the neurons. While neuronal progenitors migrate tangentially, newly born neurons undertake radial migration. Additionally, during tangential migration neurons do not rely on glial support but often associate with other neurons, hence it is referred to as neuronophilic migration (Figure 1.1, Anderson et al. (1997), Tamamaki et al. (1997), Wichterle et al. (1999), Métin et al. (2008)).

Inhibitory interneurons undergo yet another type of migration, termed chain migration. It occurs from the subventricular zone to the olfactory bulb in the rostral migratory stream and provides new GABAergic interneurons throughout life (Luskin, 1993, Alvarez-Buylla, 1997). While migrating towards the olfactory bulb, these neurons form a chain surrounded by tubular structures that consist of glial cells (Figure 1.1, Wichterle et al. (1997)).

Apart from the cerebral cortex and olfactory bulb, neuronal migration also occurs in the hippocampus and cerebellar cortex. In the latter, cerebellar granule neurons (CGNs) migration continues way into the postnatal period (Altman, 1972). During development CGNs migrate both tangentially and radially. CGN progenitors originating from the upper rhombic lip (also: germinal trigone) within rhombomere 1 (Altman, 1972, Wingate and Hatten, 1999), first undertake dorsorostral migration to reach the external granule layer (EGL, Figure 1.1). There, newly generated postmitotic neurons migrate tangentially until they change their migratory direction and continue to move radially along Bergmann glia, eventually arriving in the internal granule layer (IGL). In contrast to projection neurons in the cerebral cortex, CGNs are generated adjacent to the pial surface and migrate inwards as they mature (Figure 1.2, Altman (1972), Ryder and Cepko (1994), Wingate and Hatten (1999), Métin et al. (2008)).

Neuronal movement during migration consists of repeating cycles, each involving two events. First, the leading process dynamically extends and retracts, which results in the net elongation of the process. In the second step, which occurs after a certain delay, the soma enters the leading process, followed by a retraction of the trailing process 

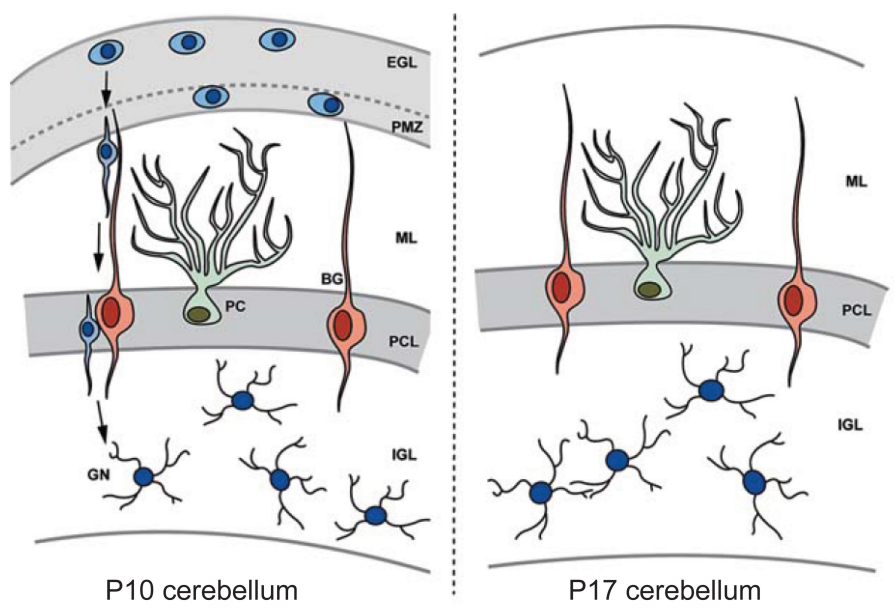

Figure 1.2.: Radial migration of newly born CGNs in the developing cerebellum. Progenitors residing in the external granule layer (EGL) generate cerebellar granule neurons (CGNs) that first accumulate in the premigratory zone (PMZ). Later, the CGNs migrate along Bergmann glia (BG) towards the internal granule layer (IGL), passing the molecular layer (ML) and the Purkinje cell layer (PCL). By the time the migration is completed, the EGL is depleted. PC - Purkinje cell. Modified from Heng et al. (2012).

\section{(Edmondson and Hatten, 1987, Komuro and Rakic, 1995).}

Proper neuronal migration results from a coordinated action of extracellular factors, such as guidance cues, growth factors, morphogenic proteins and cell adhesion molecules, acting through intracellular regulatory pathways, which include transcription factors and the ubiquitin-proteasome system that ultimately act on the cytoskeleton.

\subsubsection{Extracellular cues in neuronal migration}

Neuregulins, Netrins, Semaphorins and Slits constitute major families of guidance cues involved in neuronal migration. Here, Neuregulin 1 (NRG1) binds to the ErbB4 receptor to act as a chemoaractant for tangentially migrating interneurons (Yau et al., 2003). NRG1 is expressed in two isoforms, type III and type I/II NRG1, which differ both in their spatial distribution and their function in guiding interneurons towards the 
cortex. NRG1 type III is membrane bound and serves as a permissive substrate along the route of interneuron migration. NRG1 type I/II undergoes proteolytic cleavage and thus forms a concentration gradient directing the interneurons towards the developing pallium (Flames et al., 2004).

Apart from serving as attractive cues, these guidance proteins also function as chemorepellents. For instance, interneurons are prevented from entering the striatum by the repulsive action of Semaphorin (Sema) 3A and 3F, which bind to the Neuropilin receptors 1 and 2, respectively (Marín et al., 2001, Nóbrega-Pereira et al., 2008). Netrin1 in turn, acts via its receptor DDC (deleted in colorectal cancer) to exert a dual role in neuronal migration. While Netrin1 serves as a chemoaractant for pontine neurons migrating from the rhombic lip, it repels the cerebellar neurons from the EGL and the striatal neurons from the subventricular zone (Alcantara et al., 2000, Marin and Rubenstein, 2001).

Furthermore, growth factors, in particular BDNF (brain-derived neurotrophic factor), NT4 (Neurotrophin-4) and GDNF (glial cell line-derived neurotrophic factor) promote migration of cortical and cerebellar neurons (Polleux et al., 2002, Pozas and Ibáñez, 2005). Intracellular mechanisms involved in this regulation may include PI3K/AKT and ERK pathways (Polleux et al., 2002, Segarra et al., 2006). $\mathrm{Ca}^{2+}$-dependent autophosphorylation of TrkB receptor has been shown to be implicated in BDNF and NT-4 signaling (Behar, 1997).

Importance of the extracellular matrix components in neuronal migration was demonstrated by the reeler mutant mouse, which exhibits lamination defects accompanied by ataxia, tremor and reeling gait. Reelin, the protein mutated in this mouse, is an extracellular glycoprotein, which binds to VLDLR (Very-low-density-lipoprotein receptor) and ApoER2 (Low-density lipoprotein receptor-related protein 8). The reelin signaling requires tyrosine phosphorylation of Dab1 (Disabled-1) by nonreceptor protein kinases Src and Fyn (Arnaud et al., 2003b, Bock and Herz, 2003). 


\subsubsection{Cytoskeleton in migrating neurons}

In radially migrating neurons, microtubules exhibit a well-structured yet dynamic configuration. They form a cage-like structure surrounding the nucleus (perinuclear cage, Rivas et al. (1995)), associated with the centrosome which precedes the nucleus towards the migratory direction (Vallee et al., 2009) and provides a link to the microtubules invading the leading process (Tanaka et al., 2004, Tsai et al., 2007).

A broad array of microtubule associated proteins (MAPs) regulate tubulin dynamics. MAPs stabilize and destabilize microtubules. In addition, posttranslational modifications of both MAPs and tubulin regulate the affinity of one another (Gelfand and Bershadsky, 1991). MAP1b and Tau are structural MAPs and have been shown to regulate neuronal migration, as knockout of MAP1b or Tau in mice causes disruption of hippocampal layering and a decrease in motility of isolated cerebellar neurons (Takei et al., 2000).

Other microtubule binding proteins, like lissencephaly 1 (LIS1) and Doublecortin (DCX) have been found to be vital for proper development of the mammalian brain. Mutations in the LIS1 gene have been linked to lissencephaly (smooth brain) in humans, a condition characterized by a smooth cerebral surface, aberrant neuronal migration, severe mental retardation as well as seizures, ultimately resulting in death during early childhood (Reiner et al., 1993, Ross et al., 1997). Doublecortin (DCX) also controls migration of cortical neurons (Bai et al., 2003) and mutations in the DCX gene are associated with X-linked lissencephaly as well as double cortex syndrome (Gleeson et al., 1998). Both LIS1 and DCX bind to microtubules to increase their stability and promote centrosome coupling to the nucleus during neuronal migration (Tsai et al., 2007, Moores et al., 2004, Solecki et al., 2009).

During neuronal migration, certain proteins and organelles have to be transported from and to the distal parts of the neurons. The intracellular transport is carried out by molecular motors. Cytoplasmic dynein, a minus end-directed microtubule motor protein, has been shown to be essential for the centrosomal and nuclear translocation but not for the extension of the leading process of migrating neurons (Tsai et al., 2007).

MAPs constitute important regulators of molecular motors. Dynein activity is mod- 
ulated by proteins like LIS1, NDE1 and NDEL1 (Vallee et al., 2009). LIS1 activates cytoplasmic dynein, which is localized at the leading process and exerts a pulling force on microtubules and the centrosome (Tsai et al., 2007). In contrast to the centrosomal translocation, nuclear movement might also be mediated by a LIS1 and dynein that localize to the nuclear envelope and associate with microtubules in the perinuclear cage (Tsai et al., 2007). Among others, RanBP2 (RAN binding protein 2), SUN1 and 2 (Sad1 and UNC84 domain containing 1 and 2), Syne-1 and 2 (spectrin repeat containing, nuclear envelope 1) have been suggested to mediate the connection of dynein to the nuclear envelope (Splinter et al., 2010, Zhang et al., 2009b).

Besides the microtubules, the integrity of the actin cytoskeleton is crucial for neuronal migration, considering that suppression of actin polymerization results in inhibition of neuronal movement (Rivas et al., 1995). Consistent with this finding, disruption of actin function underlies several neurodevelopmental disorders associated with defective migration, such as bilateral periventricular nodular heterotopia (Fox et al., 1998, de Wit et al., 2009), classic lissencephaly (Kholmanskikh et al., 2003, 2006) or lissencephaly with cerebellar hypoplasia (D’Arcangelo et al., 1995, Schmid et al., 2005).

Major regulators of the actin cytoskeleton are Rho GTPases, which are either in an active, GTP-bound or inactive, GDP-bound state. Active Rho GTPases are able to bind effector proteins and modulate the cytoskeleton. Three members of the Rho GTPase family RhoA, Rac1 and Cdc42 have been extensively characterized in neuronal development (Govek et al., 2011). Processes like interkinetic nuclear migration, cycling of cortical neurons progenitors and soma translocation during radial migration in the cortex require actin depolymerization (Chai et al., 2009), which is regulated by actindisassembling proteins like cofilin and Rho GTPases.

\subsubsection{The Centrosome during neuronal development}

The centrosome or microtubule organizing center (MTOC), is believed to be the main site of microtubule nucleation in the cell (Baas et al., 2005a, Higginbotham and Gleeson, 2007). Various proteins involved in neuronal migration, like DISC1, NDEL1, LIS1 or DCX localize to the centrosome (Morris et al., 2003, Sasaki et al., 2000, Feng et al., 2000, 
Tanaka et al., 2004), establishing the MTOC as an essential cellular structure in migrating neurons. During migration, movement of the centrosome into the leading process precedes the translocation of the nucleus (Solecki et al., 2004, Tanaka et al., 2004, Vallee et al., 2009). This observation prompted the hypothesis that the centrosome transfers the pulling force of the microtubules in the leading process to the perinuclear cage, enabling the movement of the nucleus. Evidence by Solecki and colleagues revealed that overexpression of PAR6 $\alpha$ (part of the PAR3-PAR6-aPKC polarity complex) in neurons causes disruption of the perinuclear cage as well as inhibition of centrosomal movement and neuronal migration (Solecki et al., 2004). On the other hand, migrational analysis in cerebellar slices uncovered that the nuclear translocation along the microtubules can also occur independently of the centrosomal position relative to the nucleus. This finding suggests an additional, acentrosomal force acting on the nucleus in migrating neurons (Umeshima et al., 2007).

\subsubsection{DISC1 - a converging point of the signaling pathways in neuronal migration}

Disrupted in Schizophrenia 1 (DISC1) is considered to be a risk gene for schizophrenia and related psychiatric disorders (Millar et al., 2000b, Ekelund et al., 2001, Hennah et al., 2003). Since its identification, several studies have described the role of DISC1 in various neurodevelopmental processes, including the regulation of neural progenitor proliferation, neurite outgrowth and neuronal migration (Mao et al., 2009, Kamiya et al., 2006, 2005). Since no enzymatic activity has been ascribed to DISC1 until now, it is believed to exert its function by interacting with a plethora of proteins, many of which are cytoskeletal regulators.

\subsubsection{DISC1 as a susceptibility gene for schizophrenia}

DISC1 was discovered during a study a Scottish family suffering from mental disorders. In this family, a balanced translocation between chromosomes 1 and 11 was found to co-segregate with schizophrenia, major depression and bipolar disorder (St

8 
Clair et al., 1990, Millar et al., 2000a, 2001). The aforementioned translocation disrupts the DISC1 gene and an antisense non-coding RNA, DISC2 gene (Millar et al., 2000a). Meanwhile, various linkage and association studies confirmed the relationship between the DISC locus and several psychiatric disorders (Ekelund et al., 2000, Hwu et al., 2003, Ekelund et al., 2004). On the other hand, many studies showed no correlation (Sanders et al., 2008, Houlihan et al., 2009, Okuda et al., 2010) however the examined SNPs also varied between the studies, so the link of variations in the DISC1 gene to major mental disorders remains under debate.

\subsubsection{Localization of DISC1}

Human DISC1 transcript has been detected in various neural and non-neural tissues, with the highest expression in placenta, heart and brain (Millar et al., 2000b). In rodents, the most abundant expression is detectable in the heart, with weaker expression in the brain, kidney, liver and testis (Ma et al., 2002, Ozeki et al., 2003).

Within the human brain, DISC1 is highly expressed in the dentate gyrus (Lipska et al., 2006, James et al., 2004), but lower expression has been also detected in the temporal and parahippocampal cortex (Lipska et al., 2006). High levels of mouse DISC1 mRNA and protein are found in the dentate gyrus, while lower levels have been reported in cerebellum, cerebral cortex, ammon's horn, olfactory bulbs, paraventricular and arcuate nuclei of the hypothalamus and amygdala (Ma et al., 2002, Miyoshi et al., 2003). Prominent expression in the dentate gyrus supports the possibility that DISC1 constitutes a genetic risk factor for schizophrenia, since the hippocampus has been associated with the pathology of this disease (Csernansky et al., 2002, Lawrie et al., 1999, Friston et al., 1992, Venables, 1992, Kerwin et al., 1988).

In humans, DISC1 is upregulated during prenatal and neonatal period, as well as during puberty (Lipska et al., 2006). Expression of mouse DISC1 peaks at E13.5 when the neurons are generated and at P35, when the animals enter puberty (Schurov et al., 2004). This expression profile indicates that DISC1 may play an important role in neurodevelopmental processes (Ozeki et al., 2003, Lipska et al., 2006, Schurov et al., 2004, Austin et al., 2004). 
At the subcellular level, DISC1 localizes to mitochondria (Ozeki et al., 2003, James et al., 2004), the centrosome (Kamiya et al., 2005, 2006) and the cytoplasm, where it colocalizes with cytoskeletal components like F-actin (Miyoshi et al., 2003), $\alpha$-Tubulin (Brandon et al., 2004, 2005), MAP2 (Brandon et al., 2005) and gelsolin (James et al., 2004). In cultured primary neurons and neuronal cell lines, DISC1 expression has been detected in the cell body, nucleus, neurites and growth cones (Ozeki et al., 2003, James et al., 2004, Ogawa et al., 2005, Brandon et al., 2005, Shinoda et al., 2007, Taya et al., 2007). Interestingly, the subcellular localization of DISC1 undergoes changes during cell differentiation. In rodent PC12 cells and SH-SY5Y, a human neuroblastoma cell line, DISC1 translocates from the cytoplasm to the processes as the cells differentiate (Ozeki et al., 2003, James et al., 2004).

Immunohistochemistry of human cortical slices demonstrated that DISC1 localizes to the cell bodies and apical dendrites of pyramidal neurons and glial cells. Furthermore, DISC1 was detected in axon terminals, postsynaptic density, dendritic spines, ribosomes and dendritic shafts (Sawa and Roberts, 2006).

\subsubsection{Genomic and protein structure of DISC 1}

Four alternative transcripts of human DISC1 have been detected until now. The Long splice form (L) consists of 13 exons, the Long variant (Lv) form results from skipping 66 distal nucleotides of exon 11, the Short (S) form utilizes a 3'UTR of intron 9, producing an alternative exon 9a, and the Extremely short (Es) form skips the splicing donor site of exon 3 and includes 2 codons from intron 3 before using an alternative STOP codon. In mouse, two splice variants are known so far: the first one contains at least exons 1-8 and the second one corresponds to the human Es form (Taylor et al., 2003).

Although no crystal structure of DISC1 is available, the human full length protein has been predicted to contain an N-terminal "head" domain, encompassing amino acids 1-350, corresponding to exons 1-2 and a C-terminal "tail" domain, consisting of amino acids 351-854, which corresponds to exons 3-13 (Millar et al., 2000b, 2001). The Nterminal fragment accommodates a predicted nuclear localization signal and a short alpha-helix motif (Bord et al., 2006, Ma et al., 2002, Taylor et al., 2003). The C-terminal 
fragment in turn is far more conserved and contains several predicted coiled-coil domains, mediating protein-protein interactions (Taylor et al., 2003).

\subsubsection{DISC1 in regulation of neuronal migration}

DISC1 is involved in the control of early stages neuronal migration, and acts as a molecular switch between neuronal proliferation and the initiation of migration. Two mechanisms involved in this regulation have been identified so far. On the one hand, the interaction of DISC1 with nonphosphorylated Dixdc1 (DIX domain containing 1) favors neural progenitor proliferation via the WNT/GSK3 $\beta$ signaling. On the other hand, the phosphorylation of Dixdc1 by Cdk5 (cyclin-dependent kinase 5) promotes DISC1 binding to NDEL1. Dixdc1 interaction with DISC1 and NDEL1 has been shown to be indispensable for neuronal migration, as the knockdown of Dixdc1 or disruption of DISC1-Dixdc1 binding leads to disturbed migration in cortical neurons (Singh et al., 2010). Phosphorylation of DISC1 at S710 constitutes another mechanism that triggers the transition from proliferation to migration. Non-phosphorylated DISC1 preferentially binds GSK3 $\beta$ and thus regulates neural progenitor proliferation. S710phosphorylated DISC1 in turn, interacts with BBS1 and BBS4 to promote migration (Ishizuka et al., 2011a).

The role of DISC1 in the control of neuronal migration differs depending on the developmental stage and the brain region. While DISC1 RNAi inhibits migration of the cortical neurons (Kamiya et al., 2005) and of the granule neurons in the deveploping hippocampus (Meyer and Morris, 2009), it does not influence migration of pyramidal neurons in the developing CA1 hippocampal region (Meyer and Morris, 2009). However another group observed that migration of CA1 pyramidal neurons at later stages was disturbed by DISC1 downregulation (Tomita et al., 2011). In contrast, DISC1 knockdown in the adult hippocampus causes excessive migration of the dentate gyrus neurons (Duan et al., 2007). Regulation of migration in the adult dentate gyrus seems to be mediated by the Akt pathway and the actin-binding protein Girdin (Kim et al., 2009). Additionally, DISC1 has been implicated in the regulation of adult dentate gyrus neuron migration mediated by the NMDA (N-methyl-D-aspartate) receptor pathway (Namba 
et al., 2011).

A major site of DISC1 activity to regulate neuronal migration is the centrosome. DISC1 is required for the centrosomal localization of CAMDI (coiled-coil protein associated with myosin II and DISC1), which regulates proper positioning of the centrosome during neuronal migration (Fukuda et al., 2010). Additionally, DISC1 has been found to interact with APP (Amyloid Precursor Protein) and this interaction is necessary for DISC1 centrosomal localization (Young-Pearse et al., 2010). Furthermore, APP and DISC1 seem to cooperate in the regulation of neuronal migration, since migration defects caused by the knockdown of APP can be rescued by DISC1 overexpression (Young-Pearse et al., 2007, 2010). Moreover, interaction of DISC1 and BBS4 (BardetBiedl syndrome 4) is essential for recruiting PCM1 (pericentriolar material 1) to the centrosome and thus for the regulation of neuronal migration (Kamiya et al., 2008). Furthermore, the genetic variants of DISC1 S740C and L607F disturb the centrosomal localization of PCM1 (Eastwood et al., 2010, 2009).

DISC1 has been also implicated in regulation of microtubule structure and function. A microtubule spin down assay using SH-SY5Y cells demonstrated that DISC1 is found in the microtubule fraction and binds to microtubule associated proteins, like MIPT3 (Microtubule-Interacting Protein Associated With TRAF3) or MAP1A (Morris et al., 2003). Along this line, overexpression of DISC1 leads to a disrupted microtubule network and to the accumulation of polymerized microtubules at the cell periphery (Kamiya et al., 2005). DISC1 also associates with the microtubule motor proteins: the plus end-directed kinesin-1 and the minus end-directed dynein (Kamiya et al., 2005, Shinoda et al., 2007). Since motor proteins are involved in the transport of cellular cargo, it is not surprising that DISC1 is required for the transport of e.g. Grb2 (growth factor receptor-bound protein 2) and Girdin along the axons to the growth cones (Enomoto et al., 2009, Shinoda et al., 2007, Taya et al., 2007). Girdin is essential for neuronal migration and morphogenesis, since its overexpression in the adult dentate gyrus leads to excessive migration of the newly born neurons, which become mispositioned in the outer granule cell layer and molecular layer (Kim et al., 2009). Furthermore, the Girdin knockout mouse exhibits defects in the mossy fiber pathfinding and misplaced neurons 
in the dentate gyrus (Enomoto et al., 2009).

\subsubsection{Interaction of NDEL1 and DISC1 in developing brain}

NDEL1 (nuclear distribution element-like 1) is an important regulator of neural development and one of the best characterized binding partners of DISC1. Processes like cytoskeletal organization, intracellular transport, membrane trafficking, enzymatic reactions (harboring oligopeptidase activity), and cell signaling (by interacting with Cdc42) have been attributed to NDEL1 function (Nguyen et al., 2004, Shu et al., 2004, Liang et al., 2004, 2007, Hayashi et al., 2005, Guo et al., 2006, Kamiya et al., 2006, Mori et al., 2007, 2009, Shen et al., 2008, Shim et al., 2008, Toth et al., 2008, Yamada et al., 2008, Ma et al., 2009, Zhang et al., 2009a). Additionally, several studies implicated NDEL1 in neuronal migration (Sasaki et al., 2005) and demonstrated that the interaction of DISC1 and NDEL1 is required in this event. Consistently with this finding, migration defects caused by the knockdown of DISC1 are further enhanced by knockdown of NDEL1 RNAi, suggesting a functional interaction of both proteins (Duan et al., 2007).

The DISC1-NDEL1 complex controls neuronal migration mainly by interacting with cytoskeletal regulators. One of the mechanisms of NDEL1 action involves binding to dynein and LIS1 (Torisawa et al., 2011). NDEL1 acts to activate dynein motility by directly interacting with the molecular motor, resulting in its dissociation from the microtubules (Torisawa et al., 2011). Additionally, LIS1 acts as an inhibitor when bound to dynein and its interaction with NDEL1 releases this inhibition, which also contributes to dynein activation (Torisawa et al., 2011, Żyłkiewicz et al., 2011).

During migration, NDEL1 binds to LIS1 and dynein on the nuclear envelope and the subsequent activation of the molecular motor causes retrograde nuclear transport towards the centrosome in the migratory direction. Consistent with that, NDEL1 knockdown increases the distance between the nucleus and the centrosome, which results in the neurons stalled in the ventricular and subventricular zones (Shu et al., 2004).

Not only microtubules but also the actin cytoskeleton is regulated by NDEL1, e.g. by regulating the activity of small GTPases. NDEL1 sequesters Cdc42 GAP (Cdc41 GTPaseactivating protein) that inactivates $\mathrm{Cdc} 42$. This results in the accumulation of active 
Cdc42 at the leading edge, which in turn is essential for proper neuronal migration (Shen et al., 2008).

Additionally, NDEL1 harbors endo-oligopeptidase activity, and processes neuropeptides including neurotensin (Camargo et al., 1983, Hayashi et al., 2005). Interessingly, the interaction with DISC1 inhibits the enzymatic activity of NDEL1, which may lead to altered levels of neuropeptides in the brain (Hayashi et al., 2005).

\subsubsection{Axon specification and extension}

Axon specification in vivo is closely related to neuronal migration. During radial migration, cortical and hippocampal pyramidal neurons form two kind of processes. A leading process, which precedes the cell body responding to guidance cues and eventually gives rise to dendrites, and a trailing process that forms the axon (Rakic, 1971, 1972, Shoukimas and Hinds, 1978).

Newly born CGNs migrating tangentially along the molecular layer, also form a leading and a trailing process on either side of the cell body, however both processes will eventually become an axon. As CGNs initiate radial migration towards the IGL, a third process is formed, perpendicular to the first two and becomes the new leading process. The two processes created initially during tangential migration, fuse to generate a T-shaped axon, anchored in the ML and connected to the migrating cell body. Fasciculated axons in the ML form so called parallel fibers (Altman and Bayer, 1997, Ramon y Cajal, 1995, Kawaji et al., 2004).

In vitro axon specification has been extensively studied in dissociated neuronal cultures. Cultured neurons transition through well-defined stages, from an unpolarized cell to a neuron with distinct axonal and dendritic domains (Fig. 1.3, Dotti et al. (1988)).

The tip of a growing axon is equipped with a highly specialized structure, the growth cone, which constantly explores the environment, senses extracellular cues and responds to them with a directional movement (Bentley and O'Connor, 1994, Tanaka and Sabry, 1995, Suter and Forscher, 2000, Song and Poo, 2001). Growth cones consist of two main domains, the peripheral domain (P-domain) and the central domain (C-domain), distinct in their shape and their molecular composition. The P-domain 


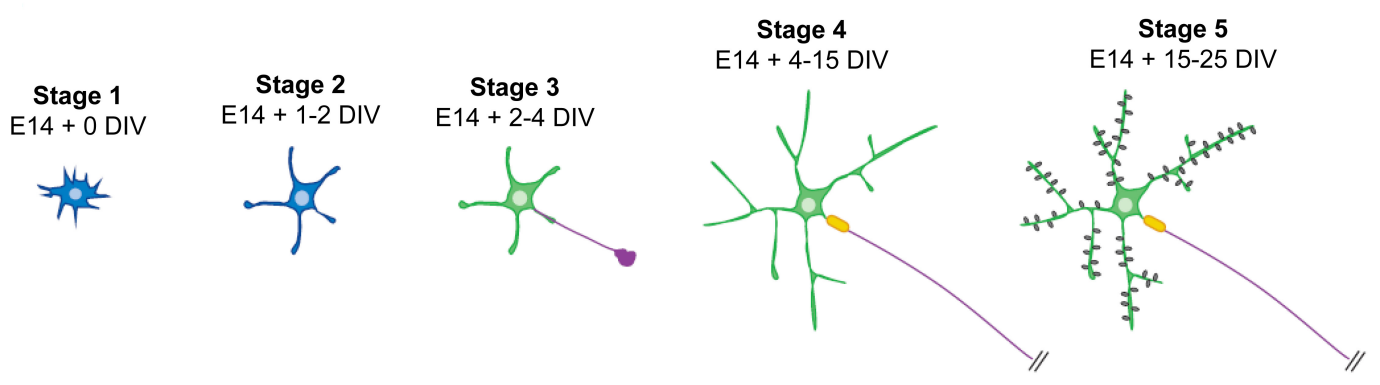

Figure 1.3.: Stages of cortical neuron polarization in vitro. Stage 1: dissociated cortical neurons form filopodia and lamellipodia-like protrusions. Stage 2: several indistinguishable neurites are formed. Stage 3: one of the processes starts rapid outgrowth and acquires axonal characteristics. Stage 4: axon and dendrites elongate and branch rapidly. Stage 5: mature neuron with dendritic spines and functional synapses. Modified from Barnes and Polleux (2009).

contains dynamic, finger-shaped filopodia protruding from flattened edges of lamellipodia. Filopodia and lamellipodia are rich in actin and invaded by single dynamic microtubules (Bridgman and Dailey, 1989, Letourneau, 1983). The C-domain in turn, is less dynamic but characterized by intensive microtubule polymerization and organelle transport (Dent and Gertler, 2003).

Axon elongation can be divided into three steps: protrusion, engorgement and consolidation. First, filopodia and lamellipodia are generated, which is driven mainly by polymerization of actin. In the second step, vesicles and organelles are transported to the growth cone. Finally, during the consolidation step, the proximal part of the growth cone is forming an extension of the axon and organelle transport becomes bidirectional (Goldberg and Burmeister, 1986).

\subsubsection{Regulation of neuronal morphogenesis}

During neuronal morphogenesis, extracellular cues and intracellular signaling pathways ensure proper axon pathfinding and wiring of the brain. Extracellular signals include neurotrophins, for instance NGF (nerve growth factor), BDNF (brain-derived neurotrophic factor), NT-3 (neurotrophin-3), and NT-4/5 (neurotrophin-4/5Bibel and Barde 
(2000)). Additionally, molecules like semaphorins (Pasterkamp and Kolodkin, 2003), netrins (Kennedy, 2000), Slits (Brose and Tessier-Lavigne, 2000) and ephrins (Kullander and Klein, 2002), as well as sonic hedgehog (Shh) (Charron et al., 2003), bone morphogenetic proteins (BMPs) (Butler and Dodd, 2003), and Wingless-type (Wnt) (Lyuksyutova et al., 2003) proteins serve as guidance cues for growing axons.

Extracellular cues together with their neuronal receptors act to mediate attractive and repulsive responses. Thus, regulation of receptor expression provides a mechanism to control axon growth and guidance. Another level of control is ensured by intracellular pathways relaying extracellular signals to the cytoskeleton. Most of these pathways involve Rho GTPases, but recently DISC1 emerged as a promising candidate to integrate cytoskeletal rearrangements and extracellular signaling.

Cytoskeletal dynamics directly drive morphological changes in developing neurons. During initial neurite outgrowth, local microtubule stabilization has been shown to specify which one of the yet unpolarized processes will become the axon (Witte et al., 2008). Furthermore, Tanaka and colleagues demonstrated that the dynamic behavior of microtubules is indispensable for axon elongation (Tanaka et al., 1995). While axon pathfinding depends mainly on the actin cytoskeleton, there is growing evidence that microtubules are involved in this process as well. Microtubule orientation in the growth cone has been shown to be an indicator of the future axon outgrowth direction (Sabry et al., 1991, Tanaka and Kirschner, 1995). Axon turning is yet another event controlled by microtubules, as local manipulations of microtubule dynamics using caged drugs causes the growth cone to change the direction (Buck and Zheng, 2002).

Several studies demonstrated that structural MAPs are essential for neuronal morphogenesis. For instance, MAP2 has been implicated in dendrite outgrowth, as its loss causes reduction of dendritic length (Harada et al., 2002). Additionally, MAP1b and Tau localize to the growth cone and regulate neuronal migration as well as neurite outgrowth (Takei et al., 2000, 1997, Gonzalez-Billault et al., 2002, DiTella et al., 1996).

The phosphorylation status of MAPs greatly modifies their microtubule-binding properties and is essential for regulating neuronal morphogenesis and migration. For instance, MAP1b and Tau, phosphorylated by GSK3 $\beta$ (glycogen synthase kinase $3 \beta$ ), 
change the stability of the microtubules and influence neuronal polarization (GonzalezBillault et al., 2004, Sperbera et al., 1995). Spatial regulation of GSK3 $\beta$ activity (low in axons, high in dendrites) ensures precise control of neuronal polarization (Jiang et al., 2005, Oinuma et al., 2007). Another MAP regulated by GSK3 $\beta$ is APC (adenomatous polyposis coli), a protein localized to the tip of a growing axon. Its phosphorylation by GSK3 $\beta$ results in loss of APC binding to microtubules. As a consequence, the destabilized microtubules cause growth cone pausing (Purro et al., 2008). Moreover, GSK3 $\beta$ mediated phosphorylation of CRMP-2 (collapsin-response mediator protein 2), abundantly present at axon tips, prevents it from stabilizing microtubules. Neurotrophin-3 inhibits GSK3 $\beta$, which in turn causes CRMP-2 dephosphorylation resulting in axon growth stimulation (Yoshimura et al., 2005).

Additionally, actin cytoskeleton regulation by Rho GTPases plays an important role in neuronal morphogenesis. Cdc42 in its GTP-bound state is known to bind and activate N-WASP (neuronal Wiskott-Aldrich syndrome protein). The activated N-WASP can then promote filopodia formation via Arp2/3 (actin-related protein 2/3), which has actin-polymerizing activity. In contrast, Rac1 is involved in lamellipodia formation by binding to WAVE1, which also stimulates Arp2/3 activity (Goley and Welch, 2006, Pak et al., 2008). Stimulation of filopodia and lamellipodia formation by Cdc42 and Rac1, respectively, promotes neurite extension (Nobes and Hall, 1995, Ridley et al., 1992). Additionally, RhoA binds to its effector mDia, which in turn interacts with profilin to induce localized actin polymerization and focal adhesion formation (Watanabe et al., 1997). Interaction with mDia mediates the axon growth-promoting effect of RhoA (Arakawa et al., 2003). However, RhoA also acts to inhibit axon growth, utilizing a pathway that includes binding to its other effector ROCK (Govek et al., 2005).

\subsubsection{DISC1 in neuronal morphogenesis}

DISC1 function in neuronal morphogenesis is mainly related to the centrosome, the growth cone and the axon, where it facilitates the transport of cellular cargo. Centrosomal DISC1 interacts among others with pericentrin (PCNT), which is necessary for its function in regulating microtubule nucleation and aster formation (Shimizu et al., 
2008). Pericentrin is essential for proper neural development, since mutations in this gene are associated with a form of dwarfism exhibiting reduced brain size (Griffith et al., 2008, Rauch et al., 2008). Centrosomal PCM1 plays an important role in recruitment of pericentrin to the centrosome, which is a prerequisite for proper microtubule organization (Dammermann and Merdes, 2002). DISC1 together with BBS4 recruit PCM1 to the centrosome (Kamiya et al., 2008), which in turn is essential for axonal morphogenesis (de Anda et al., 2010) and embryonic neurogenesis (Ge et al., 2010).

Furthermore, interaction with FEZ1 (fasciculation and elongation protein zeta-1), is believed to mediate the neurite growth-promoting effect of DISC1 overexpression in PC12 cells. This hypothesis is consistent with the increased binding of DISC1 and FEZ1 during neuritogenesis in these cells. It is also bolstered by the finding that overexpression of the FEZ1-binding domain of DISC1 has a dominant negative effect on neurite extension. Modulation of the cytoskeleton at the growth cone may be a possible mechanism of neurite outgrowth regulation by both proteins (Miyoshi et al., 2003).

Another interacting partner of DISC1 implicated in neurite outgrowth is DBZ (DISC1Binding Zinc-finger protein). Hattori and colleagues found that, similar to FEZ1, overexpression of DISC1-interacting region of DBZ inhibits neurite elongation in PC12 cells and in hippocampal neurons (Hattori et al., 2007).

In growing axons, DISC1 cooperates with kinesin to transport various cargo proteins to the growth cones. For instance Grb2 has been shown to be transported by DISC1kinesin complex and its accumulation at the growth cone depends on the interaction with DISC1. Additionally, DISC1 and Grb2 binding is required for neurotrophin (NT-3)mediated axon extension (Shinoda et al., 2007). Another cargo transported by kinesin in a DISC1-dependent manner in the LIS1-NDEL1-14-3-3 $\varepsilon$ complex. Axon elongation depends on DISC1-mediated anterograde transport of the NDEL1/LIS1/14-3-3 $\varepsilon$ complex by kinesin-1, since knockdown of DISC1 abrogates this transport and inhibits axon growth (Taya et al., 2007). Moreover, overexpression of a DISC1 mutant, which fails to bind to NDEL1 causes defects in neurite outgrowth in PC12 cells (Kamiya et al., 2006).

Selective stabilization of microtubules, which is required for axonogenesis is also connected to NDEL1 translocation to the axon hillock, resulting from its phosphorylation 
at serine 251 by Aurora-A kinase (Mori et al., 2007).

LIS1 and NDEL1 also form a complex with NDE1 (nuclear distribution protein nudE homolog 1). DISC1 has been shown to connect the LIS1/NDEL1/NDE1 complex to the activity of PDE4 (Phosphodiesterase 4), which controls the local levels of cAMP. It has been demonstrated that NDE1 is phosphorylated at T131 by PKA (protein kinase A) to modulate its interaction with LIS1 and NDEL1. PKA is activated by cAMP and phosphorylates NDE1 in a DISC1-PDE4 dependent manner. Overexpression of NDE1 mutant that mimics the T131 phosphorylation, inhibits neurite outgrowth in rat myeloma NS-1 cells indicating that DISC1 modulates the NDE1 function in neuronal morphogenesis (Bradshaw et al., 2011).

\subsection{Ubiquitin-proteasome system in neural development}

In recent years, the ubiquitin-proteasome system (UPS) has emerged as one of the major regulators of extracellular and intracellular signaling in developing neurons. For instance, ubiquitin-proteasome-mediated degradation of DAB1 is essential for reelin signaling, which regulates the actin cytoskeleton (Arnaud et al., 2003a) and defective DAB1 ubiquitination causes excessive migration of neurons (Feng et al., 2007, Simó et al., 2010). Furthermore, loss of netrin-1 responsiveness after midline crossing may be mediated by proteasomal degradation of the netrin receptor DCC (Kim et al., 2005, Hu et al., 1997). Additionally, Cdh1-APC ubiquitinates FBXO31 in a proteolytic manner to restrict the axon growth (M. Vadhvani, unpublished data). FBXO31 in turn, mediates the ubiquitination of PAR6 $\alpha$ in the control of axon growth (Vadhvani et al., 2013).

Also small GTPases are regulated by ubiquitination. SMURF1 and SMURF2 ubiquitinate inactive RhoA and Rap1B respectively to regulate neuronal polarity (Wang et al., 2003, Schwamborn et al., 2007). Additionally, active Rap2 is ubiquitinated by NEDD4, which results in promoting the dendrite growth (Kawabe et al., 2010). SMURF1 itself is ubiquitinated and targeted for proteasomal degradation by yet another ubiquitin ligase, Cdh1-APC (Kannan et al., 2012b). Cdh1-APC has been also shown to act upstream of the 
p250GAP, a Rho GAP, which stimulates axon growth (Kannan et al., 2012a). Moreover, Cdh1-APC targets the transcription factors SnoN and Id2 for proteasomal degradation in the control of axon growth (Stegmüller et al., 2006, Lasorella et al., 2006).

\subsubsection{Protein ubiquitination as a posttranslational modification}

Ubiquitination is a modification that, analogous to phosphorylation or acylation, is used by almost all eukaryotic cells to regulate protein function. Three enzymes are required to attach ubiquitin, a highly conserved 76-amino acid polypeptide, to the target protein. First, the E1 ubiquitin activating enzyme catalyzes binding of an ATP molecule to the C-terminal glycine of ubiquitin (Ciechanover et al., 1981, Hershko et al., 1981, Haas et al., 1982). Next, the activated ubiquitin is transferred to the E2 ubiquitin conjugating enzyme, by the formation of a thioester bond. Finally, the E3 ubiquitin ligases mediate the covalent attachment of ubiquitin to a lysine on the substrate (Hershko et al. (1983), Fig. 1.4). However, ubiquitination of cysteine, serine and threonine residues have also been reported (Cadwell and Coscoy, 2005, Ravid and Hochstrasser, 2007, Wang et al., 2007).

Traditionally, proteasomal degradation has been considered the only outcome of protein ubiquitination. However, over the recent years, our understanding of possible ubiquitination functions has dramatically increased. Ubiquitination can regulate transcription, endocytosis, protein interactions and protein activity (Hicke, 2001). For comprehensive list of ubiquitination functions, see Fig. 1.5.

The number of attached ubiquitin moieties and the way they are linked to each other constitutes a code, that determines the fate of a ubiquitinated protein. A substrate is monoubiquitinated if a single ubiquitin molecule is attached to it. Attaching single ubiquitins at several position on one substrate is called multimonoubiquitination. Polyubiquitination is a process of attaching a ubiquitin chain. Such chains can be homotypic (homogeneous), when one specific lysine of ubiquitin is used to covalently attach the subsequent ubiquitin, or heterotypic (heterogeneous), with ubiquitins that are connected by various linkages in one chain. All the seven lysines (Lys6, Lys11, Lys27, Lys29, Lys33, Lys48 and Lys63) and the N-terminal Met1 (creating linear chains) 


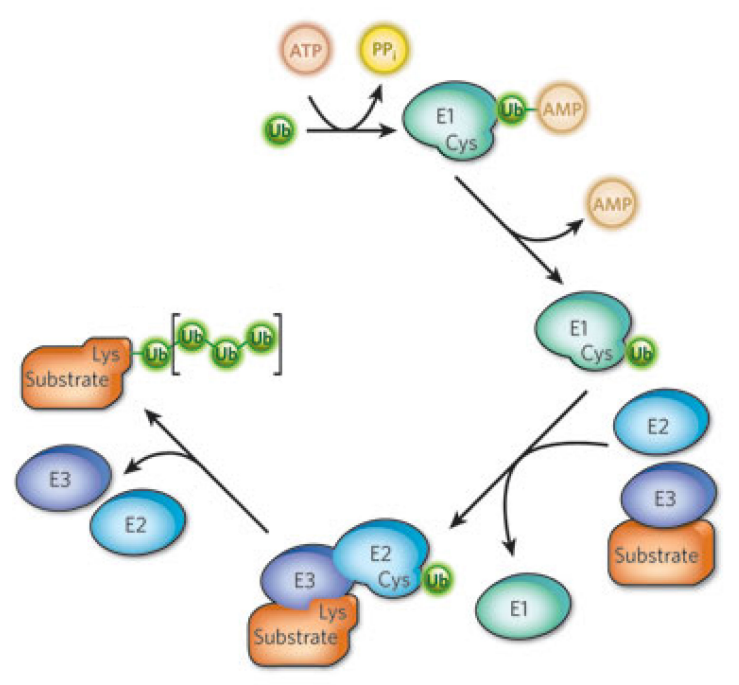

Figure 1.4.: Protein ubiquitination requires three different enzymes. Ubiquitin (Ub) activated by E1 is passed to E2 and a thioester intermediate is formed. Subsequently ubiquitin is transferred to the substrate that is recruited by E3. Modified from Hochstrasser (2009).

of ubiquitin can be used to form polyubiquitin chains (Ikeda and Dikic (2008), Behrends and Harper (2011), Komander and Rape (2012) Fig. 1.5).

\subsubsection{E3 ubiquitin ligases}

Among the three enzymes involved in the ubiquitination process, E3 ubiquitin ligases are the most numerous (around 600 in humans, as compared to two E1s and approximately $30 \mathrm{E} 2 \mathrm{~s}$ ) and the most interesting, since they confer substrate specificity (Scheffner et al., 1995). The family of ubiquitin ligases can be separated into two major classes: RING (really interesting new gene)-type and HECT (homologous to E6AP carboxyl terminus)-type E3 ubiquitin ligases. The transfer of ubiquitin to a substrate by a HECT-type ligase requires the formation of a transient thioester bond with the E3. In contrast, RING E3 ligases function as scaffolds to facilitate the transfer of ubiquitin from the E2 onto the substrate (Rotin and Kumar, 2009, Deshaies and Joazeiro, 2009). 


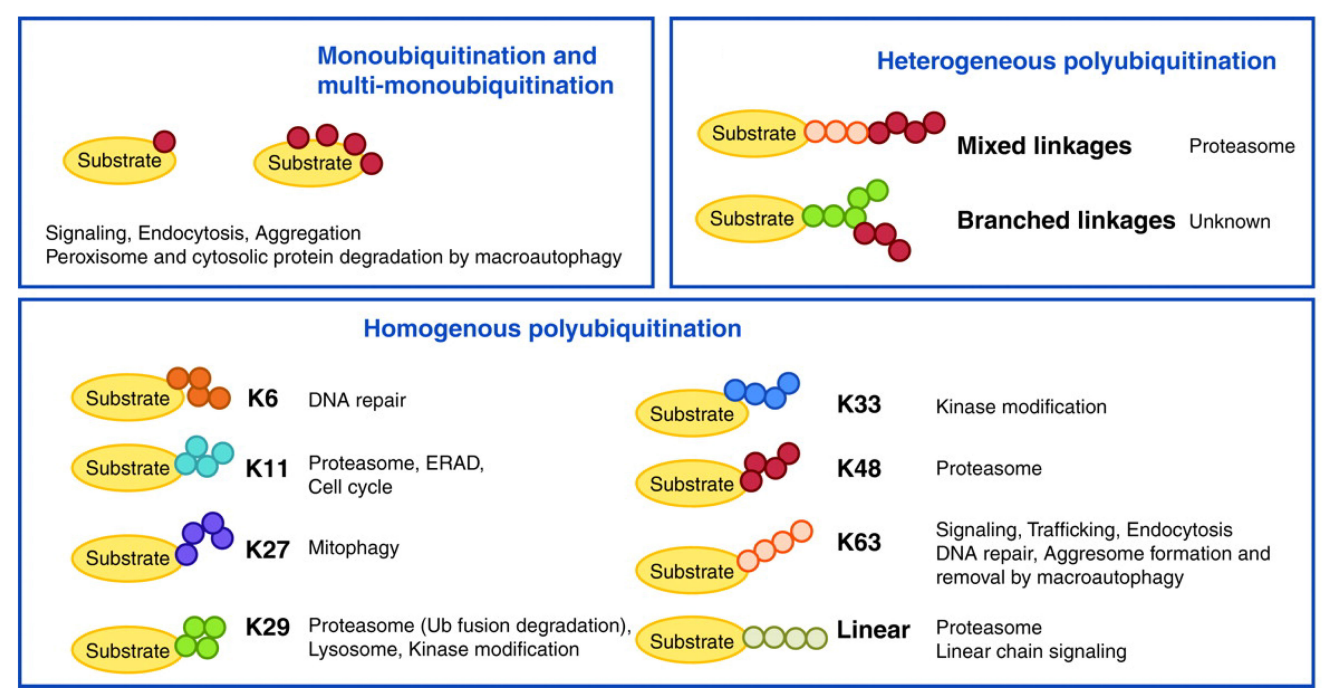

Figure 1.5.: Functions of specific modes of ubiquitination. Ubiquitin can be attached to the substrates as single moieties or chains of various configurations. Different types of ubiquitination specify the fate of the protein. Modified from Wong and Cuervo (2010).

When RING ligases are involved, the E2 enzymes specify the type of ubiquitin chain formed. In contrast, the C-terminus of HECT ligases alone determines the ubiquitin linkage type (Kim and Huibregtse, 2009).

\subsubsection{SCF complex and F-box proteins}

The SCF (Skp1, Cullin 1, F-box) complex is a conserved RING-type E3 ubiquitin ligase first identified in budding yeast (Feldman et al., 1997, Skowyra et al., 1997). It consists of four components: Skp1 (S phase kinase-associated protein 1), Cul1 (Cullin 1), F-box protein (FBP) and a RING finger-containing Rbx1 (RING-box protein 1). F-box proteins act as variable adapters, recognizing and recruiting different sets of substrates (Deshaies, 1999). Apart from the SCF complex, various other Cullin-RING ligases (CRLs) have been identified, which utilize different Cullins and substrate recruiting adapters (Fig. 1.6).

All F-box proteins (FBPs) share a common, approximately 40 amino acid motif - the 


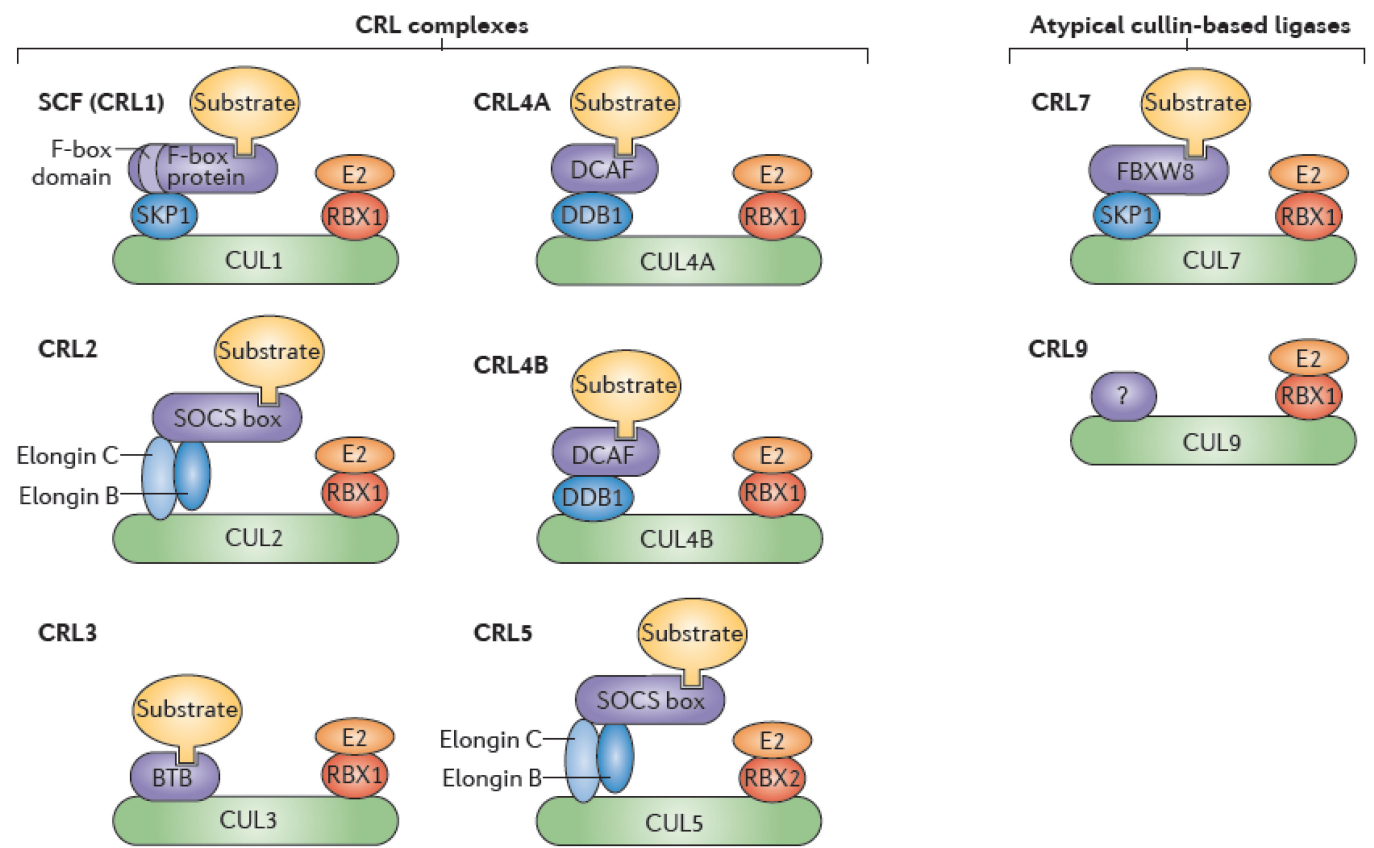

Figure 1.6.: Various Cullins with their substrate recognition adapter proteins. 8 different Cullins (CUL) have been identified until now and most of them have been shown to form CRLs (Cullin-RING ligases) analogous to the SCF (Skp1, Cullin1, F-box) complex, but with different components that fulfill the functions of F-box proteins and Skp1. Cullin 7 forms an SCF-like complex, but only one F-box protein (FBXW8) is known to interact with it so far (Sarikas et al., 2008). Modified from Skaar et al. (2013).

F-box domain, which was first identified in Cyclin F (Bai et al., 1996). FBPs have been subdivided into three groups: FBXW, FBXL and FBXO, reflecting their domains composition. FBXW proteins are characterized by a WD40 domain, Leu-rich repeat domain is present in FBXL proteins and the other FBPs have been categorized as FBXO (F-box only) proteins. Distinct domains of FBPs play different roles within the SCF complexes. The F-box domain is responsible for binding to Skp1 and Cul1, and the various additional domains are believed to serve as substrate-binding sites (Jin et al., 2004).

For some of the F-box proteins, a degron recognition motif has been characterized on their substrates (Ravid and Hochstrasser, 2008). Since several proteins can carry the 
same degron, this mechanism enables FBPs to recognize multiple targets. However, several levels of regulation ensure that the substrates are degraded in a spatially and temporally controlled manner. First of all, the best characterized FBPs recognize only phosphorylated degrons. For instance $\beta$ TRCP recognizes a motif: Asp-pSer-Gly-XaaXaa-pSer, and FBXW7 recognizes a sequence: pThr-Pro-Pro-Xaa-pSer where Xaa is any amino acid and pSer or pThr are phosphorylated serine or threonine) (Wu et al., 2003, Welcker and Clurman, 2008). In some cases a sequential phosphorylation by several kinases is required for target recognition, increasing the stringency of the process. For instance, GSK $3 \beta$ can only phosphorylate JUN when it is already phosphorylated by another kinase (Wei et al., 2005) Therefore, the availability and activation of specific kinases regulates protein ubiquitination. Several other protein modifications can also facilitate substrate recognition. For example FBXO2 binding to pre-integrin $\beta 1$ and FBXO6 binding to T cell receptor $\alpha$-chain depends on the glycosylation of the substrates (Glenn et al., 2008). Conversely, some modifications can block substrate recognition by the FBPs. FBXL2 cannot bind phosphorylated $\mathrm{p} 85 \beta$ and the binding is promoted by protein tyrosine phosphatase (PTPL1)-mediated phosphorylation (Kuchay et al., 2013).

Not all F-box proteins exert their function as part of an SCF complex, although several of these actions still involve binding to Skp1. SCF-independent functions of F-box proteins have been predominantly characterized in budding yeast, where Mfb1 and Mdm30 are involved in mitochondria fusion and tubule formation (Dürr et al., 2006). Moreover, Ctf13 interacts with Skp1 and constitutes a part of the CBF3 kinetochore binding complex (Kitagawa et al., 1999). Some F-box proteins also harbor enzymatic activity, including the helicase Fbh1, which unwinds DNA in an ATP-dependent manner (Kim et al., 2002, 2004).

\subsubsection{F-box proteins in neuronal development and disease}

The human genome encodes more than 70 F-box proteins (Jin et al., 2004), however the function of only few of them has been characterized. Most of these studies focus on the role of F-box proteins in cell cycle and thus their implications in cancer (Skaar et al., 2009, Frescas and Pagano, 2008, Welcker and Clurman, 2008, Frescas and Pagano, 2008). 
Growing evidence supports their involvement in development and function of the nervous system. FBPs have been shown to regulate various stages of neurodevelopment, from neural progenitor proliferation, neuronal migration and morphogenesis.

During neurogenesis, the degradation of REST (RE1-silencing transcription factor) by $\mathrm{SCF}^{\beta-T r c p}$ ensures proper neural progenitors differentiation (Westbrook et al., 2008). FBXW7 in turn, controls neural development by ubiquitination of Notch and c-JUN, which is essential for neural progenitor differentiation and apoptosis (Hoeck et al., 2010). Furthermore, FBXL10, which is a subunit of the PRC1 (Polycomb repressive complex 1) E3 ligase, monoubiquitination of the histone $2 \mathrm{~A}$, which is essential for proper differentiation of embryonic stem cells (Wu et al., 2013). The FBXL10-deficient mouse shows an increase in apoptosis and neural progenitor proliferation. Additionally, roughly half of these mice suffer from exencephaly and retinal coloboma resulting from a failure of neural tube closure, which causes death shortly after birth (Fukuda et al. 2011). Downregulation of the FBP, Skp1 (FBXL1), has been demonstrated to increase the proliferation of Xenopus neuronal precursors, while its overexpression decreases neurogenesis. This effect is SCF-dependent and requires the degradation of the cyclin dependent kinase inhibitor, Xic1 (Boix-Perales et al., 2007).

Neuronal morphology is also regulated by of FBPs. For instance, it has been shown that depletion of FBXW7 disrupts axonal arborization of Purkinje cells in mouse cerebellum (Jandke et al., 2011). On the other hand, dendrite morphology is regulated by FBXW8 that targets Grasp65 for proteasomal degradation and thereby regulates Golgi morphology (Litterman et al., 2011). Apart from that, downregulation of FBXO31 has been shown to inhibit neurite growth and to disturb establishment of axon-dendrite polarity (Vadhvani et al., 2013).

Additionally, the loss of FBXO45 in mice causes defects in cerebral axonal tracts and in the innervation of the diaphragm, as well as aberrant formation of neuromuscular synapses. Interestingly, the innervation defects are not caused by the inability of neurons to grow axon but rather a pathfinding defect, since the outgrowth of processes was observed in the isolated FBXO45 knockout neurons (Saiga et al., 2009).

Many of the FBPs play versatile roles in neurodevelopment, regulating its several 
aspects. For instance, knockdown of FBXO31 also inhibits migration of CGNs, which has been shown by using in vivo electroporation of postnatal rat cerebellum (Vadhvani et al., 2013). Moreover, FBXO45 knockout mice exhibit defects in radial and tangential migration in the telencephalon (Saiga et al., 2009). Additionally, knockout of FBXW7 in mice cerebellum causes premature CGN migration and mispositioning of Purkinje neurons (Jandke et al., 2011).

Apart from their role in neural development, several F-box proteins have also been implicated in pathogenesis of neuronal disorders. Mutations in FBXO7 gene have been shown to cause a form of juvenile Parkinsonism (Fonzo et al., 2009). Moreover, it has been demonstrated that the levels of the F-box protein FBXL2 decrease in the temporal but not the frontal cortex of the Alzheimer's disease (AD) patients. Interestingly, it is the temporal cortex that undergoes degeneration in $\mathrm{AD}$ (Watanabe et al., 2013). Additionally, FBXO2 has been suggested to be a possible target of Alzheimer's disease therapy, since its overexpression in an $\mathrm{AD}$ mouse model decreased the $\beta$-amyloid levels and ameliorated the synaptic defects. The underlying mechanism involved ubiquitination and proteasomal degradation of BACE1 ( $\beta$-site APP-cleaving enzyme 1) (Gong et al., 2010). Moreover, FBXO2 has been shown to act with Skp1 but independently of the SCF complex to control the inner ear function and the targeted knockout of FBXO2 in mice causes progressive hearing loss (Nelson et al., 2007).

\subsubsection{Aim of the project}

F-box proteins are not only important players in the control of cell cycle but emerged also as regulators of neurodevelopment and potential targets of therapeutic approaches (Hirota et al., 2012, Wu et al., 2012b, Orlicky et al., 2010, Aghajan et al., 2010, Sakamoto et al., 2001, 2003). Hence, it is important to learn more about novel F-box proteins with a role in brain development and disease. The goal of my thesis was to characterize the previously undescribed, CNS-specific F-box protein FBXO41 in neurodevelopment. The first aim of the study was to elucidate the expression of FBXO41 in the developing brain using biochemical and immunocytochemical methods. The second aim of the project combined in vitro and in vivo approaches to investigate the role of FBXO41 in neuronal 
migration and morphogenesis, and to identify interactors of FBXO41 to establish an FBXO41 pathway in developing neurons. 



\section{Materials and methods}

\subsection{Chemicals and kits}

Unless otherwise stated, the chemicals used in this study were purchased from Merck (Darmstadt, Germany), Sigma-Aldrich (Munich, Germany), Roth (Karlsruhe, Germany), Applichem (Darmstadt, Germany), Biomol (Hamburg, Germany), Serva Electrophoresis (Heidelberg, Germany), Invitrogen (Darmstadt, Germany), Becton Dickinson and Company (USA) and Worthington (UK).

NucleoBond ${ }^{\circledR}$ Xtra Midi EF, NucleoSpin ${ }^{\circledR}$ Plasmid QuickPure kits and NucleoSpin ${ }^{\circledR}$ Gel and PCR Clean-up kit were from Macherey-Nagel (Düren, Germany). QuikChange II Site-Directed Mutagenesis Kit was ordered from Agilent Technologies (USA), Human Fetal Brain Mate \& Plate ${ }^{\circledR}$ Library was from Clontech Laboratories, Inc. (USA).

\subsection{Antibodies}

Primary antibodies used in this study are listed in the table below. Secondary antimouse IgG and anti-rabbit IgG antibodies conjugated to fluorophores (Alexa Fluor 488 or 555) or horse radish peroxidase were bought from Dianova (Hamburg, Germany). Anti-goat IgG antibody coupled to horse radish peroxidase was from Santa Cruz Biotechnology. 


\begin{tabular}{|c|c|c|c|}
\hline Target & Host & Application & Company \\
\hline FBXO41 & rabbit & WB $(1: 250)$ & $\begin{array}{l}\text { Eurogentec } \\
\text { made) }\end{array}$ \\
\hline GFP & rabbit & WB $(1: 1000)$, ICC $(1: 1500)$ & Invitrogen \\
\hline GFP & mouse & WB $(1: 1000)$, IHC (1:100) & Santa Cruz Biotechnology \\
\hline myc & mouse & WB (1:2000), ICC (1:250) & Santa Cruz Biotechnology \\
\hline Flag & mouse & WB $(1: 1000)$, ICC $(1: 250)$ & Sigma-Aldrich \\
\hline$\beta$-galactosidase & mouse & WB (1:500) & Santa Cruz Biotechnology \\
\hline$\gamma$-Tubulin & mouse & WB $(1: 5000)$ & Sigma-Aldrich \\
\hline pan $14-3-3$ & mouse & WB $(1: 20000)$ & Santa Cruz Biotechnology \\
\hline PLP & mouse & WB $(1: 150)$ & $\begin{array}{l}\text { Prof. Klaus-Armin Nave, } \\
\text { Max Planck Institute of } \\
\text { Experimental Medicine, } \\
\text { Göttingen, Germany }\end{array}$ \\
\hline GFAP & rabbit & WB (1:500) & Promega \\
\hline DISC1 & goat & WB $(1: 500)$ & Santa Cruz Biotechnology \\
\hline NDEL1 & rabbit & WB $(1: 1000)$ & Abcam \\
\hline Cdc42 & rabbit & WB $(1: 250)$ & Cell Signaling Technology \\
\hline Katanin p60 & rabbit & WB $(1: 250)$ & Sigma-Aldrich \\
\hline MAP2 & mouse & $\operatorname{ICC}(1: 1000)$ & Sigma-Aldrich \\
\hline $\mathrm{CC} 3$ & rabbit & WB $(1: 1000)$ & Cell Signaling Technology \\
\hline total ERK1/2 & rabbit & WB $(1: 1000)$ & Promega \\
\hline phospho-ERK1/2 & rabbit & WB $(1: 1000)$ & Cell Signaling Technology \\
\hline total Akt & rabbit & WB $(1: 1000)$ & Cell Signaling Technology \\
\hline phospho-Akt & rabbit & WB $(1: 1000)$ & Cell Signaling Technology \\
\hline SnoN & rabbit & WB (1:500) & Santa Cruz Biotechnology \\
\hline Cullin 1 & mouse & WB $(1: 1000)$ & Santa Cruz Biotechnology \\
\hline Cullin7 & mouse & WB $(1: 1000)$ & Sigma-Aldrich \\
\hline NFM & mouse & WB (1:500) & Santa Cruz Biotechnology \\
\hline Caytaxin & goat & WB (1:500) & Santa Cruz Biotechnology \\
\hline
\end{tabular}




\subsection{Lab equipment}

Experiments were carried out with the following equipment: pipettes (Gilson, USA), plastic ware (Eppendorf, Germany, Sarstedt AG, Germany and Greiner Bio-One, Germany), centrifuges (Eppendorf), rocker and shaker (Heidolph, Germany), thermocycler, (Biometra, Germany), heater block (Grant Instruments, UK), spectrophotometer (GE, UK), fluorescent microscope (Eclipse Ti), inverted light microscope (Eclipse TS100) and dissection microscope (SMZ645) (Nikon, Japan).

\subsection{Enzymes}

The enzymes used in this study are listed below:

\begin{tabular}{ll}
\hline Enzyme & Company \\
\hline Restriction enzymes & New England Biolabs \\
GoTaq $^{\circledR}$ DNA polymerase & Promega \\
Pfu DNA polymerase & Thermo Scientific \\
Calf intestinal phosphatase & New England Biolabs \\
T4 DNA ligase & Fermentas \\
T4 polynucleotide kinase & NEB \\
T4 DNA polymerase & Fermentas \\
\hline
\end{tabular}

\subsection{Solutions}

Following solutions were used through the course of the study:

PBS: $137 \mathrm{mM} \mathrm{NaCl}, 10 \mathrm{mM} \mathrm{KCl}, 20 \mathrm{mM} \mathrm{Na}_{2} \mathrm{HPO}_{4}, 20 \mathrm{mM} \mathrm{KH}_{2} \mathrm{PO}_{4} \mathrm{pH} 7.4$

PBST: PBS, $0.1 \%$ Tween 20

Running buffer: $25 \mathrm{mM}$ Tris base, $190 \mathrm{mM}$ glycine, $0.1 \%$ SDS 
Transfer buffer: $20 \mathrm{mM}$ Tris base, $153 \mathrm{mM}$ glycine, $20 \%$ methanol

Upper buffer: $0.5 \mathrm{M}$ Tris- $\mathrm{HCl} \mathrm{pH}$ 6.8, $0.4 \%$ SDS

Lower buffer: $1.5 \mathrm{M}$ Tris- $\mathrm{HCl} \mathrm{pH} 8.8,0.4 \% \mathrm{SDS}$

Triton X-100 lysis buffer: $150 \mathrm{mM} \mathrm{NaCl}, 50 \mathrm{mM}$ Tris- $\mathrm{HCl} \mathrm{pH}$ 7.5, $1 \mathrm{mM}$ EDTA, $1 \%$ Triton X-100

ColP buffer: $150 \mathrm{mM} \mathrm{NaCl}, 20 \mathrm{mM}$ Tris- $\mathrm{HCl} \mathrm{pH}$ 7.4, $1 \mathrm{mM}$ EDTA, $1 \%$ Nonidet P-40, $10 \%$ glycerol

5x SDS sample buffer: $12.5 \mathrm{ml}$ upper buffer, $10 \mathrm{ml}$ glycerol, $2 \mathrm{~g}$ SDS, $1 \mathrm{ml} \beta$-mercaptoethanol, bromophenol blue in $25 \mathrm{ml} \mathrm{H}_{2} \mathrm{O}$

HHGN: 10\% 10x HBSS-Hank's buffered salt solution [-] calcium [-] magnesium, 2.5 mM HEPES pH 7.5, $35 \mathrm{mM}$ glucose, $4 \mathrm{mM} \mathrm{NaHCO}_{3}$

Cbc: BME [+] Earle's salts [-] L-Glutamine, 10\% calf serum (heat inactivated, Hyclone), $1 \%$ PSG, $25 \mathrm{mM} \mathrm{KCl}$

DnB: $500 \mu \mathrm{l}$ DNase $(2 \mu \mathrm{g} / \mu \mathrm{l}), 10 \mathrm{ml} \mathrm{BME} \mathrm{[+]} \mathrm{Earle's} \mathrm{salts} \mathrm{[-]} \mathrm{L-Glutamine}$

TDn: $250 \mu \mathrm{l}$ DNase $(2 \mu \mathrm{g} / \mu \mathrm{l}), 5 \mathrm{ml}$ HHGN, $50 \mathrm{mg}$ Trypsin (Worthington)

2x HBSS: $4 \mathrm{~g} \mathrm{NaCl}, 117.5 \mathrm{mg} \mathrm{KCl}, 95 \mathrm{mg} \mathrm{Na}{ }_{2} \mathrm{HPO}_{4} \times 7 \mathrm{H}_{2} \mathrm{O}, 675 \mathrm{mg}$ Glucose, $2.5 \mathrm{~g}$ HEPES-free acid in $250 \mathrm{ml} \mathrm{H}{ }_{2} \mathrm{O}$

Hippocampal neuron plating medium: DMEM [+] $4.5 \mathrm{~g} / 1$ glucose [+] glutamine $[+]$ pyruvate, 10\% FBS (Biochrom), 1\% PSG, $0.0125 \mathrm{mM}$ glutamate

Hippocampal neuron growth medium: Neurobasal medium, 2\% B27 supplement, $1 \%$ PSG

HEK 293T medium: DMEM [+] $4.5 \mathrm{~g} / 1$ glucose [-] glutamine [-] pyruvate, $10 \%$ FBS, $1 \%$ GlutaMAX $^{\circledR}$ 
8\% PFA: $375 \mathrm{ml}$ sterile $\mathrm{H}_{2} \mathrm{O}$ at $60^{\circ} \mathrm{C}, 40 \mathrm{~g}$ PFA, $50 \mathrm{ml} 10 \% \mathrm{PBS}, 40 \mathrm{~g}$ sucrose, set $\mathrm{pH}$ to 7.4, fill up with sterile $\mathrm{H}_{2} \mathrm{O}$ to $500 \mathrm{ml}$

Mounting medium: $6 \mathrm{~g}$ glycerol AR, $2.4 \mathrm{~g}$ Mowiol 4-88 (Calbiochem), $6 \mathrm{ml} \mathrm{H}{ }_{2} \mathrm{O}, 12$ $\mathrm{ml} 0.2 \mathrm{M}$ Tris- $\mathrm{HCl} \mathrm{pH} 8.5$

2x TAE: 80 mM Tris-acetate, 2 mM EDTA pH 8.5

2x YT (1000 ml): $16 \mathrm{~g}$ tryptone, $10 \mathrm{~g}$ yeast extract, $5 \mathrm{~g} \mathrm{NaCl}$

Tail lysis buffer: $200 \mathrm{mM} \mathrm{NaCl}, 10 \mathrm{mM}$ Tris pH 8.0, $10 \mathrm{mM}$ EDTA, 0.5\% SDS

Annealing buffer: $100 \mathrm{mM}$ potassium acetate, $30 \mathrm{mM}$ HEPES-KOH pH 7.4, $2 \mathrm{mM}$ magnesium acetate

Buffer A: $10 \mathrm{mM}$ HEPES, $10 \mathrm{mM} \mathrm{KCl,} 0.1 \mathrm{mM}$ EDTA, $0.1 \mathrm{mM}$ EGTA

Buffer A+: Buffer A, 0.1\% Nonidet P-40

NP-40 lysis buffer: $1 \mathrm{mM}$ HEPES $\mathrm{pH}$ 7.2, 0.5\% NP-40, $0.5 \mathrm{mM}$ magnesium chloride, $0.1 \% \beta$-mercaptoethanol

\subsection{Expression constructs and primers}

Constructs 1-6 and 13-20 were cloned by Dr. J. Stegmüller, 9, 10 - by N. SchwedhelmDomeyer, 8 - by C. Mukherjee and 22 - by D. Brockelt. For details concerning generation of plasmids - see Section 2.7 


\begin{tabular}{|c|c|c|c|c|}
\hline \multicolumn{2}{|c|}{ No. Construct } & \multirow{2}{*}{$\frac{\text { Species }}{\text { mouse }}$} & \multirow[t]{2}{*}{ Primers } & \multirow{2}{*}{$\begin{array}{l}\text { Restriction sites } \\
\text { EcoRI, BamHI }\end{array}$} \\
\hline 1 & pEGFP-C2-FBXO41 & & & \\
\hline 2 & pEGFP-C2-FBXO41 aa35-873 & mouse & & EcoRI, BamHI \\
\hline 3 & pEGFP-C2-FBXO41 aa317-873 & mouse & & EcoRI, BamHI \\
\hline 4 & pEGFP-C2-FBXO41 aa355-873 & mouse & & EcoRI, BamHI \\
\hline 5 & pEGFP-C2-FBXO41 aa594-873 & mouse & & EcoRI, BamHI \\
\hline 6 & pEGFP-C2-FBXO41 aa1-546 & mouse & & EcoRI, BamHI \\
\hline \multirow[t]{2}{*}{7} & pEGFP-C2-FBXO41 $\Delta \mathrm{CTR}$ & mouse & $18031 / 17768 /$ & EcoRI, BamHI \\
\hline & & & $17769 / 18032$ & \\
\hline \multirow[t]{2}{*}{8} & pEGFP-C2-FBXO41 $\Delta$ F-box & mouse & 19426/ & EcoRI, BamHI \\
\hline & & & 19427 & \\
\hline 9 & pCMV-myc-FBXO41 & mouse & $18556 / 18552$ & KpnI, BglII \\
\hline 10 & pCMV-myc-FBXO41 $\Delta$ CTR & mouse & $18556 / 18552$ & KpnI, BglII \\
\hline 11 & pCMV-myc-FBXO41-Res & mouse & $19036 / 19037$ & KpnI, BglII \\
\hline 12 & pCMV-myc-FBXO41-Res $\Delta$ CTR & mouse & $19036 / 19037$ & KpnI, BglII \\
\hline 13 & pBluescript-U6-FBXO41 RNAi\#1 & rat, mouse & $14211 / 14212$ & ApaI, EcoRI \\
\hline 14 & pBluescript-U6-FBXO41 RNAi\#2 & rat, mouse & $14213 / 14214$ & ApaI, EcoRI \\
\hline 15 & pBluescript-U6-FBXO41 RNAi\#4 & rat, mouse & $16102 / 16103$ & ApaI, EcoRI \\
\hline 16 & pBluescript-U6-FBXO41 RNAi\#5 & rat, mouse & $16104 / 16105$ & ApaI, EcoRI \\
\hline 17 & pBluescript-U6-FBXO41 RNAi\#6 & $\begin{array}{l}\text { rat, mouse, } \\
\text { human }\end{array}$ & $20264 / 20265$ & ApaI, EcoRI \\
\hline 18 & pBluescript-U6-FBXO41 RNAi\#8 & $\begin{array}{l}\text { rat, mouse, } \\
\text { human }\end{array}$ & $20268 / 20269$ & ApaI, EcoRI \\
\hline 19 & FBXO41 RNAi\#5-CMV-EGFP & rat, mouse & $16104 / 16105$ & SacI, SacII \\
\hline 20 & pCMV-myc-Skp1 & rat & $15454 / 15455$ & XhoI, NotI \\
\hline 21 & pCMV-myc-DISC1 & human & $22773 / 22774$ & \\
\hline 22 & pBluescript-U6-DISC1 RNAi & $\begin{array}{l}\text { rat, mouse, } \\
\text { human }\end{array}$ & $17497 / 17498$ & ApaI, EcoRI \\
\hline 23 & DISC1 RNAi-CMV-EGFP & $\begin{array}{l}\text { rat, mouse, } \\
\text { human }\end{array}$ & $17497 / 17498$ & SacI, SacII \\
\hline
\end{tabular}


Expression plasmids listed below were obtained from external sources:

\begin{tabular}{lll}
\hline Construct & Species & Obtained from \\
\hline myc-FBXO31 & human & David Callen, University of Adelaide \\
pcDNA 3.1-Flag-DISC1 & human & Kirsty Millar, University of Edinburgh \\
Flag-Cullin7 & & Zhen-Qiang Pan, The Mount Sinai School \\
& & of Medicine, New York \\
pIRES-hrGFP-1a-Flag-NDEL1 & human & Minh Dang Nguyen, University of Cal- \\
& & gary \\
\hline
\end{tabular}

Following primers were used for cloning of expression constructs:

\begin{tabular}{|c|c|}
\hline Number & Sequence 5'-3' \\
\hline \multirow[t]{2}{*}{14211} & AGGCCCAGAAGCTGTTTGAGTTCAAGCTTCTCAAACAGCTTCTGG \\
\hline & GCCTCTTTTG \\
\hline \multirow[t]{2}{*}{14212} & AATTCAAAAGAGGCCCAGAAGCTGTTTGAGAAGCTTGAACTCAAA \\
\hline & CAGCTTCTGGGCCT \\
\hline \multirow[t]{2}{*}{14213} & AGGAGGTGGAGCAGAAGATCTTCAAGCTTGATCTTCTGCTCCACCT \\
\hline & ССТСТTTTG \\
\hline \multirow[t]{2}{*}{14214} & AATTCAAAAGAGGAGGTGGAGCAGAAGATCAAGCTTGAAGATCTT \\
\hline & СТGCTCCACCTCCT \\
\hline 15454 & ATTACTCGAGTAATGCCTACGATAAAGTTGCAGAGTTC \\
\hline 15455 & TTAAGCGGCCGCTCACTTCTCTTCACACCACTGGTT \\
\hline \multirow[t]{2}{*}{16102} & AGAAGGAAAGCAAGGAGTATAAGTTAACGATACTCCTTGCTTTCCT \\
\hline & ТСТСТTTTG \\
\hline \multirow[t]{2}{*}{16103} & AATTCAAAAGAGAAGGAAAGCAAGGAGTATCGTTAACTTATACTC \\
\hline & CTTGCTTTCCTTCT \\
\hline \multirow[t]{2}{*}{16104} & AAGATTCTGCACATCAAGGTAAGTTAACGACCTTGATGTGCAGAAT \\
\hline & СТTCTTTTG \\
\hline \multirow[t]{2}{*}{16105} & AATTCAAAAGAAGATTCTGCACATCAAGGTCGTTAACTTACCTTGA \\
\hline & TGTGCAGAATCTT \\
\hline
\end{tabular}




\begin{tabular}{ll}
17497 & GCAGGAGGTCAGCAAGGCCTTGAAGTTAACGCAAGGCCTTGCTGA \\
17498 & CCTCCTGCCTTTTG \\
& AATTCAAAAGGCAGGAGGTCAGCAAGGCCTTGCGTTAACTTCAAG \\
17768 & CCCACCTCCTCTGCCCAGGCTACAAGGACCAGGGCCAGGTCCG \\
17769 & CGGACCTGGCCCTGGTCCTTGTAGCCTGGGCAGAGGAGGTGGG \\
18031 & ATCGAATTCATGGCCTCGCTGGACCTACCGTACCG \\
18032 & ATCGGATCCTTAGCAGCCACCTTCTACCTTGATGTGCAGAATCTTC \\
18552 & ATCCTCGAGTTAGCAACCACCTTCTACCTT \\
18556 & ATCGAATTCTTATGGCCTCGCTGGACC \\
19036 & GAGGCCTGGCTTCTCGAAAATCCTCCATATCAAGGTAGAAGGTGG \\
19037 & CCACCTTCTACCTTGATATGGAGGATTTTCGAGAAGCCAGGCCTC \\
19426 & TATTTGGAACAGACTCGGGCATTAGCTCGCATCTTGAGGATTT \\
19427 & TATAAATCCTCAAGATGCGAGCTAATGCCCGAGTCTGTTCCA \\
20264 & AGCCACACCTACGAGACGCTAAGTTAACGAGCGTCTCGTAGGTGTG \\
& GCTCTTTTG \\
20265 & AATTCAAAAGAGCCACACCTACGAGACGCTCGTTAACTTAGCGTCT \\
1596 & CGTAGGTGTGGCT \\
20268 & AGCTGCTACTGCCGTGCCCTAAGTTAACGAGGGCACGGCAGTAGC \\
& AGCTCTTTTG \\
\hline & AATTCAAAAGAGCTGCTACTGCCGTGCCCTCGTTAACTTAGGGCAC \\
& GGCAGTAGCAGCT \\
\hline
\end{tabular}




\subsection{Molecular biology methods}

\subsubsection{General molecular cloning}

For cloning into an expression vector, a desired cDNA sequence was amplified using primers listed in Section 2.6. The PCR reaction was performed according to tables below. Annealing temperature $(\mathrm{X})$ was chosen $5^{\circ} \mathrm{C}$ lower than the melting temperature of the primers.

\begin{tabular}{ll}
\hline \multicolumn{1}{c}{ Reaction setup } \\
\hline Volume & Reagent \\
\hline $1 \mu \mathrm{l}$ & forward primer \\
$1 \mu \mathrm{l}$ & reverse primer \\
$1 \mu \mathrm{l}$ & template DNA $(100 \mathrm{ng} / \mu \mathrm{l})$ \\
$5 \mu \mathrm{l}$ & 10x reaction buffer $+\mathrm{Mg}$ \\
$0.6 \mu \mathrm{l}$ & dNTPs $(25 \mathrm{mM})$ \\
$0.5 \mu \mathrm{l}$ & Pfu DNA polymerase $(2.5$ units $/ \mu \mathrm{l})$ \\
$40.9 \mu \mathrm{l}$ & $\mathrm{H}_{2} \mathrm{O}$ \\
\hline
\end{tabular}

\begin{tabular}{|c|c|c|}
\hline \multicolumn{2}{|c|}{ PCR conditions } & \\
\hline Temperature & Time & \\
\hline $95^{\circ} \mathrm{C}$ & $3 \mathrm{~min}$ & \\
\hline $95^{\circ} \mathrm{C}$ & $30 \mathrm{~s}$ & \\
\hline $\mathrm{X}^{\circ} \mathrm{C}$ & $30 \mathrm{~s}$ & 30 cycles \\
\hline $72^{\circ} \mathrm{C}$ & $1 \mathrm{~min} / 500 \mathrm{bp}$ of template & \\
\hline $72^{\circ} \mathrm{C}$ & $10 \mathrm{~min}$ & \\
\hline $16^{\circ} \mathrm{C}$ & $\infty$ & \\
\hline
\end{tabular}


PCR product was electrophoretically separated on an agarose gel to assess its size and quality. DNA fragment of a correct size was subsequently excised from the gel, purified with the NucleoSpin ${ }^{\circledR}$ Gel and PCR Clean-up kit and eluted in $40 \mu \mathrm{l}$ of water.

The entire purified PCR product (insert) and $3 \mu \mathrm{g}$ of target expression vector were digested with restriction enzymes listed in Section 2.6, in a volume of $50 \mu \mathrm{l}$. Digested vector was additionally treated for 1 hour with 10 units of calf intestinal phosphatase (CIP) to remove the 5' phosphate group and prevent religation. Following CIP treatment, the vector was subjected to electrophoresis on an agarose gel to separate the linearized fraction, which was then extracted with the NucleoSpin ${ }^{\circledR}$ Gel and PCR Clean-up kit and eluted in $40 \mu \mathrm{l}$ water. The insert was purified with the same kit, directly after digestion. $28 \mu \mathrm{l}$ of water was used for insert elution.

Subsequent ligation of insert $(5 \mu \mathrm{l})$ and vector $(1 \mu \mathrm{l})$ was performed using 5 units of T4 DNA ligase over night at $16^{\circ} \mathrm{C}$. The next day, the entire volume of the reaction was used to transform chemically competent $\mathrm{DH} 5 \alpha$ strain of E.coli. After addition of the DNA mixture, the bacteria were incubated on ice for $20 \mathrm{~min}$, following by a $1 \mathrm{~min}$ heat shock at $42^{\circ} \mathrm{C}$. Next, bacteria were cooled down on ice for $2 \mathrm{~min}$ and $500 \mu \mathrm{l}$ of room temperature $2 \mathrm{x}$ YT media without antibiotics was added. Bacteria were then incubated with shaking at $37^{\circ} \mathrm{C}$ for 1 hour, spun down at $4000 \mathrm{rpm}$ for $5 \mathrm{~min}$ and plated on prewarmed 2x YT agar plates containing appropriate antibiotic.

The result of the ligation was assessed by digesting the mini-preparation of plasmids (obtained using the NucleoSpin ${ }^{\circledR}$ Gel and PCR Clean-up kit) with the enzymes used for cloning and by sequencing of positive clones.

\subsubsection{Generation of vector-based shRNA}

ShRNA generated for this study were in pBluescript vector containing a U6 promoter. Oligonucleotides used to clone the shRNA are listed in Section 2.6. $2 \mu \mathrm{l}$ of the sense and antisense oligonucleotide $(50 \mathrm{pmol} / \mu \mathrm{l})$ were first diluted in $46 \mu \mathrm{l}$ annealing buffer and incubated at $95^{\circ} \mathrm{C}$ for $4 \mathrm{~min}$, at $72^{\circ} \mathrm{C}$ for $10 \mathrm{~min}$ and finally cooled down to room temperature at a rate of $0.1^{\circ} \mathrm{C} / \mathrm{min} .20 \mu \mathrm{l}$ of the reaction mixture was phosphorylated using polynucleotide kinase (PNK). At the same time, pBluescript-U6 vector was di- 
gested with ApaI, treated with T4 DNA polymerase to obtain blunt ends following by digestion with EcoRI and dephosphorylation by calf intestinal phosphatase. Vector and annealed oligonucleotides were ligated over night at $4^{\circ} \mathrm{C}$. Positive clones were identified by digestion with $\mathrm{HpaI}$ (introduced oligonucleotides contained the appropriate restriction site) and verified by sequencing.

\subsubsection{Site-directed mutagenesis}

Rescue constructs for this study were generated by site-directed mutagenesis using the QuickChange ${ }^{\circledR}$ Site-Directed Mutagenesis Kit (Agilent Technologies) according to the manufacturer instructions. First, the PCR reaction was performed as shown in the following tables (primer sequences are provided in Section 2.6):

\begin{tabular}{ll}
\hline & \multicolumn{1}{c}{ Reaction setup } \\
\hline Volume & Reagent \\
\hline $1,5 \mu \mathrm{l}$ & forward primer $(10 \mathrm{pmol} / \mu \mathrm{l})$ \\
$1,5 \mu \mathrm{l}$ & reverse primer $(10 \mathrm{pmol} / \mu \mathrm{l})$ \\
$1 \mu \mathrm{l}$ & plasmid DNA $(10 \mathrm{ng} / \mu \mathrm{l})$ \\
$5 \mu \mathrm{l}$ & 10x reaction buffer $[+] \mathrm{Mg}$ \\
$1 \mu \mathrm{l}$ & dNTPs $(25 \mathrm{mM})$ \\
$1 \mu \mathrm{l}$ & Pfu DNA polymerase $(2.5$ units $/ \mu \mathrm{l})$ \\
$40 \mu \mathrm{l}$ & $\mathrm{H}_{2} \mathrm{O}$ \\
\hline
\end{tabular}

PCR conditions

\begin{tabular}{ll|l}
\hline Temperature & Time \\
\hline $95^{\circ} \mathrm{C}$ & $30 \mathrm{~s}$ & \\
$95^{\circ} \mathrm{C}$ & $30 \mathrm{~s}$ & 18 cycles \\
$55^{\circ} \mathrm{C}$ & $1 \mathrm{~min}$ & \\
$68^{\circ} \mathrm{C}$ & $1 \mathrm{~min} / 500 \mathrm{bp}$ of template & \\
\hline
\end{tabular}


Next, the reaction mix was incubated on ice for 2 min and digested with DpnI to remove the non-mutated parental DNA strand. $1 \mu \mathrm{l}$ of the digested sample was used to transform the DH5 $\alpha$ E.coli, which were subsequently plated on prewarmed 2x YT agar plates. Obtained clones were subjected to sequencing to validate successful mutagenesis.

\subsubsection{Generation of deletion mutants ( $\Delta \mathrm{CTR}$ and $\Delta \mathrm{F}$-box $)$}

Deletion mutants used in this study were generated by performing two separate PCR reactions to amplify the fragment form the START codon until just before the deletion domain and from just after the deletion domain until the STOP codon. In the second step, a final PCR was performed to fuse both fragments, resulting in a protein lacking a specific region. Fusion of both fragments was possible because the primers harbor a sequence generating complementary overhangs on both sides of the domain to be deleted.

Reaction setup to generate the $\mathrm{N}$ - and $\mathrm{C}$ - terminal fragments and fusion PCR setup are shown in the following tables (for the PCR conditions see Subsection 2.7.1):

\begin{tabular}{ll}
\hline \multicolumn{2}{c}{ PCR for N-and C-terminal fragments } \\
\hline Volume & Reagent \\
\hline $1 \mu \mathrm{l}$ & forward primer $(10 \mathrm{pmol} / \mu \mathrm{l})$ \\
$1 \mu \mathrm{l}$ & reverse primer $(10 \mathrm{pmol} / \mu)$ \\
$100 \mathrm{ng}$ & template DNA \\
$5 \mu \mathrm{l}$ & 10x reaction buffer $[+] \mathrm{Mg}$ \\
$0.6 \mu \mathrm{l}$ & dNTPs $(25 \mathrm{mM})$ \\
$0.5 \mu \mathrm{l}$ & Pfu DNA polymerase $(2.5$ units $/ \mu \mathrm{l})$ \\
$40.9 \mu \mathrm{l}$ & $\mathrm{H}_{2} \mathrm{O}$ \\
\hline
\end{tabular}

40 


\begin{tabular}{ll}
\hline & \multicolumn{1}{c}{ Fusion PCR } \\
\hline Volume & Reagent \\
\hline $1 \mu \mathrm{l}$ & forward primer $(10 \mathrm{pmol} / \mu \mathrm{l})$ \\
$1 \mu \mathrm{l}$ & reverse primer $(10 \mathrm{pmol} / \mu \mathrm{l})$ \\
$1 \mu \mathrm{l}$ & N-terminal fragment \\
$1 \mu \mathrm{l}$ & C-terminal fragment \\
$5 \mu \mathrm{l}$ & 10x reaction buffer $[+] \mathrm{Mg}$ \\
$0.6 \mu \mathrm{l}$ & dNTPs $(25$ mM $)$ \\
$0.5 \mu \mathrm{l}$ & Pfu DNA polymerase $(2.5$ units $/ \mu \mathrm{l})$ \\
$40.9 \mu \mathrm{l}$ & $\mathrm{H}_{2} \mathrm{O}$ \\
\hline
\end{tabular}

\subsection{Cell culture}

\subsubsection{Culture of HEK 293T cells}

HEK 293T cells were maintained in $10 \mathrm{~cm}$ dishes in DMEM supplemented with $10 \%$ FBS and 1\% GlutaMAX ${ }^{\circledR}$. When approx. 90\% confluent, cells were split. Therefor the cells were first washed with PBS and incubated with $2 \mathrm{ml} 1 \mathrm{x}$ TE (10 mM Trypsin pH 8.0 and $1 \mathrm{mM}$ EDTA) for $5 \mathrm{~min}$ at $37^{\circ} \mathrm{C}$. Subsequently, $8 \mathrm{ml}$ of the HEK 293T medium was added to inactivate the trypsin and the cells were detached from the plate by pipetting the liquid up and down. Cell suspension was centrifuged at $4{ }^{\circ} \mathrm{C}, 800 \mathrm{rpm}$ for 5 min, resuspended with HEK 293T medium and plated on 6-well plates for biochemical experiments or $10 \mathrm{~cm}$ plates for maintaining the cell line.

\subsubsection{Culture of primary cerebellar granule neurons}

Cerebella were isolated from 6 days old Wistar rats or from P5 C57/BL6 mice and collected into the HHGN medium kept on ice. Fine forceps were used to remove the meninges under a dissection microscope. Next, the cerebella were washed 3 times with HHGN and incubated with $4 \mathrm{ml}$ of TDn for $10 \mathrm{~min}$ at $37^{\circ} \mathrm{C}$ and another $10 \mathrm{~min}$ at RT. Digested tissue was again subjected to $3 \mathrm{HHGN}$ washes and triturated in $4 \mathrm{ml}$ of $\mathrm{DnB}$ 
until the suspension appeared homogenous. Following $5 \mathrm{~min}$ incubation on ice, the supernatant was transferred to a fresh conical tube. The remaining tissue was triturated again with another $4 \mathrm{ml}$ of $\mathrm{DnB}$ and combined with the first part of the supernatant. The cell suspension was centrifuged at $4^{\circ} \mathrm{C}, 800 \mathrm{rpm}$ for $5 \mathrm{~min}$ and the pellet was resuspended in $20 \mathrm{ml}$ of $\mathrm{Cbc}$ medium. Neurons were counted in a hemocytometer and plated in Cbc medium on plates or coverslips (12 mm, Thermo Fisher) coated with polyornithine. For coating the plates or coverslips, a $15 \mu \mathrm{g} / \mathrm{ml}$ polyornithine solution was applied for $30 \mathrm{~min}$ at $37^{\circ} \mathrm{C}$, followed by 2 washes with sterile $\mathrm{H}_{2} \mathrm{O} .20 \times 10^{6}$ neurons were plated on 24 -well plates, $30 \times 10^{6}$ on 6-well plates and $50 \times 10^{6}$ on $10 \mathrm{~cm}$ plates. On DIV 1, a mitotic inhibitor (Arabinofuranosyl Cytidine, Ara-C) was added directly to the culture medium at a final concentration of $10 \mu \mathrm{M}$. Every $3 \mathrm{rd}$ day the medium was supplemented with $35 \mathrm{mM}$ glucose.

\subsubsection{Culture of primary hippocampal neurons}

Hippocampi of E18 Wistar rat embryos were isolated in HBSS ([-] calcium [-] magnesium, Gibco) under the dissection microscope and collected in the HBSS medium on ice. Following 3 washes with HBSS the volume was reduced to $1.8 \mathrm{ml}$ and $200 \mu \mathrm{l}$ of TE $\left(0.05 \%\right.$, Gibco) was added. The hippocampi were incubated at $37^{\circ} \mathrm{Cfor} 10 \mathrm{~min}$. Next, the tissue was again washed $3 \mathrm{x}$ with HBSS and triturated in $1 \mathrm{ml}$ of hippocampal neuron plating medium with freshly added $50 \mu \mathrm{l}$ DNase $(2 \mathrm{mg} / \mathrm{ml})$. Cell suspension was spun down at $4^{\circ} \mathrm{C}, 800 \mathrm{rpm}$ for $5 \mathrm{~min}$ and neurons were plated at a density of $50000 /$ well in a 24 -well plate with coverslips. Coverslips were pre-coated with $0.00083 \%$ poly-L-lysine for $2 \mathrm{~h}$ at $37^{\circ} \mathrm{C}$, washed $2 \mathrm{x}$ with $\mathrm{H}_{2} \mathrm{O}$ and incubated with HBSS until needed. One day after plating the medium was changed to the hippocampal neuron growth medium. 


\subsection{Calcium phosphate transfection}

\subsubsection{Transfection of HEK 293 T cells}

HEK 293T cells were transfected in 6-well plates at a confluency of approx. 70-80\%. For transfecting of 1 well, plasmid DNA was diluted in water to the volume of $90 \mu \mathrm{l}$. Next, $10 \mu \mathrm{l} 2.5 \mathrm{M}$ calcium chloride was added and mixed well by pipetting, followed by addition of $100 \mu \mathrm{l}$ of $2 \mathrm{x}$ HBSS. The mixture was then incubated at RT for $5 \mathrm{~min}$ and added to the cells.

\subsubsection{Transfection of primary neurons}

Cerebellar granule neurons and hippocampal neurons were transfected in 24-well plates. First, the neurons were washed twice with DMEM [+] $4.5 \mathrm{~g} / \mathrm{l}$ glucose [-] glutamine [-] pyruvate and incubated with this solution for 30-45 min. The conditioned culture medium was collected and stored at $37^{\circ} \mathrm{C}$ for further use. In the meantime, the DNA precipitates were prepared. For 1 well, DNA was diluted in water to a volume of $18 \mu \mathrm{l}$ and $2 \mu \mathrm{l}$ of $2.5 \mathrm{M}$ calcium chloride as well as $20 \mu \mathrm{l} 2 \mathrm{x}$ HBSS were added. After 20 min incubation, DNA precipitates were added to the neurons. 5-18 minutes later, neurons were washed twice with DMEM [+] $4.5 \mathrm{~g} / 1$ glucose [-] glutamine [-] pyruvate and the conditioned culture medium was replaced.

\subsection{Immunocytochemistry}

HEK 293T cells or primary neurons were washed twice with PBS and fixed with 4\% PFA for 10 minutes. After another 2 washes with PBS, cells were permeabilized for 10 min with $0.4 \%$ Triton X-100 diluted in PBS. Subsequently the permeabilizing solution was removed by washing twice with PBS and a blocking buffer was applied (BME $+10 \%$ horse serum) for $30 \mathrm{~min}$. In the next step, the cells were incubated either for $1 \mathrm{~h}$ at RT or overnight at $4^{\circ} \mathrm{C}$ with the primary antibody diluted in the blocking buffer. After 2 washes with the blocking buffer, the secondary, fluorophore-coupled antibody diluted 
in blocking buffer was added and incubated for 30-45 min. After 2 PBS washes, the cells were incubated for 5 min with a DNA dye (bisbenzimide Hoechst 33258 or 4',6diamidino-2-phenylindole - DAPI), washed twice with PBS and mounted with Mowiol mounting medium on microscope slides (Marienfeld). Unless otherwise stated, all steps were performed at RT.

\subsection{Morphometrical analysis}

Cerebellar granule neurons or hippocampal neurons were transfected with 1-2 $\mu \mathrm{g}$ of expression plasmid, $0.3 \mu \mathrm{g}$ of transfection marker (pEGFP C1 vector) and $0.4 \mu \mathrm{g}$ Bclxl (antiapoptotic protein). 3 (for the gain-of-function studies) or 4 (for the loss-of-function experiments) days following the transfection, neurons were fixed and stained with the GFP antibody as described in Section 2.10. Images of at least 30 neurons were acquired in a blinded manner using fluorescent microscope Eclipse Ti and NIS-Element software (Nikon). Axon length and total dendrite length were measured by manual tracing of the processes using Fiji (NIH) software. Dendrite complexity was assessed with a Sholl Analysis plugin of ImageJ (NIH).

\subsection{Biochemical methods}

\subsubsection{Tissue lysate preparation}

Mouse or rat tissues were isolated from animals sacrificed by decapitation or $\mathrm{CO}_{2}$ inhalation. Tissues were then placed in ice-cold Triton X-100 lysis buffer with freshly added protease inhibitors ( $1 \mathrm{mM} \mathrm{DTT}, 1 \mu \mathrm{g} / \mathrm{ml}$ pepstatin, $3 \mu \mathrm{g} / \mathrm{ml}$ aprotinin and $1 \mu \mathrm{g} / \mathrm{ml}$ leupeptin) and mechanically disrupted using $2 \mathrm{ml}$ Dounce homogenizer or tissue dispersing instrument (Ultra Turrax, IKA ${ }^{\circledR}$ ). Following $30 \mathrm{~min}$ incubation on ice, the samples were centrifuged for $10 \mathrm{~min}$ at $13000 \mathrm{rpm}$, at $4^{\circ} \mathrm{C}$ and the supernatant was either used for further experiments or flash-frozen in liquid nitrogen. 


\subsubsection{Preparation of cell lysates}

Culture medium was aspirated from HEK 293T cells or primary neurons growing in culture plates and the cells were washed with PBS. Next, the Triton X-100 lysis buffer freshly supplemented with protease inhibitors ( $1 \mathrm{mM} \mathrm{DTT}, 1 \mu \mathrm{g} / \mathrm{ml}$ pepstatin, $3 \mu \mathrm{g} / \mathrm{ml}$ aprotinin and $1 \mu \mathrm{g} / \mathrm{ml}$ leupeptin) was added, the cells were removed from the culture dish using a cell scraper and collected in a reaction tube. Samples were incubated on ice for $30 \mathrm{~min}$ and centrifuged for $10 \mathrm{~min}$ at $13000 \mathrm{rpm}$, at $4^{\circ} \mathrm{C}$.

\subsubsection{Bradford assay}

The concentration of proteins in tissue or cell lysates was determined using Bradford assay, where protein binding to Coomassie Brilliant Blue G-250 causes its absorbance shift. Bovine serum albumin - BSA $(4,6,8$, and $10 \mu \mathrm{g}$ for a standard curve) or a protein of interest was added to $1 \mathrm{ml}$ of the Bradford reagent (Bio Rad) diluted 5x in PBS. Samples were mixed and the absorbance was measured at the wave length of $595 \mathrm{~nm}$. Protein concentration was calculated based on the BSA standard curve.

\subsubsection{SDS-PAGE and western blot}

Polyacrylamide gels used for SDS-PAGE were prepared in a gel casting system from Bio-Rad according to the following table:

\begin{tabular}{ll}
\hline Reagent & Separating gel (10\%)/Stacking gel (3.9\%) \\
\hline $30 \%$ acrylamide & $5 / 0.65 \mathrm{ml}$ \\
Lower buffer/upper buffer & $3.75 / 1.25 \mathrm{ml}$ \\
$\mathrm{H}_{2} \mathrm{O}$ & $6.25 / 3.05 \mathrm{ml}$ \\
APS & $50-60 / 30 \mu \mathrm{l}$ \\
TEMED & $5-6 / 2-3 \mu \mathrm{l}$ \\
\hline
\end{tabular}

Cell or tissue lysates containing 30-100 $\mu$ g proteins were mixed with the 5x SDS sample buffer and incubated at $95^{\circ} \mathrm{C}$ for $5 \mathrm{~min}$. After loading on the polyacrylamide gel, 
the samples were subjected to electrophoresis at $35 \mathrm{~mA}$ per gel. Next the proteins were transferred onto a nitrocellulose membrane by a wet transfer at $300 \mathrm{~mA}$ for $90 \mathrm{~min}$. Following the transfer, the membrane was blocked with $4 \%$ milk powder in PBST for 30 min at RT. Subsequently, 3 PBST washes, 10 min each, were applied and the membrane was incubated with a primary antibody for $1 \mathrm{~h}$ at RT or overnight at $4{ }^{\circ} \mathrm{C}$. Primary antibodies were diluted either in $3 \%$ BSA with $0.02 \% \mathrm{NaN}_{3}$ or in $4 \%$ milk powder in PBST. After 3 more PBST washes, the membrane was incubated for $30 \mathrm{~min}$ at RT with a secondary antibody coupled to horseradish peroxidase (HRP), diluted in PBST supplemented with $4 \%$ milk powder. The membrane was then subjected to 3 final PBST washes and incubated shortly with the enhanced chemiluminescent (ECL) horse radish peroxidase substrates (Thermo Scientific, Bonn). Next, an X-ray film was exposed to the membrane and developed in a dark room.

\subsubsection{Co-Immunoprecipitation (CoIP)}

For coimmunoprecipitation experiments, transfected HEK 293T cells were lysed as described in Section 2.12.2 but the CoIP buffer was used instead of the Triton X 100 lysis buffer. $10 \%$ of the samples was set aside as an input control and the remaining lysates were rotated with an approx. $1 \mu \mathrm{g}$ of an appropriate antibody for $4 \mathrm{~h}$ or overnight at $4^{\circ} \mathrm{C}$. Subsequently, $20 \mu \mathrm{l}$ of protein A-sepharose beads were added to every sample and rotated at $4^{\circ} \mathrm{C}$ for another hour. Protein A-sepharose beads used for this step were first washed twice with the CoIP buffer and stored in equal amount of CoIP buffer at $4^{\circ} \mathrm{C}$ until used. Following incubation with protein A-sepharose beads, the samples were spun down at $10000 \mathrm{rpm}$ for $30 \mathrm{~s}$ and the supernatant was removed. Remaining beads with bound proteins were washed 3x with the Triton X-100 lysis buffer and once with PBS, next the PBS was removed leaving approx. $10 \mu \mathrm{l}$ of the liquid inside and the samples were incubated at $95^{\circ} \mathrm{C}$ for 5 min with $30 \mu \mathrm{l}$ of SDS sample buffer. The immunoprecipitates and inputs were further used for SDS-PAGE and western blot. 


\subsubsection{Subcellular fractionation}

CGNs from 2 wells of a 6-well plate were washed with PBS and lysed with buffer A supplemented with protease inhibitors (0.5 mM PMSF, $5-10 \mu \mathrm{g} / \mathrm{ml}$ aprotinin and 5 mM N-ethyl maleimide (NEM)). Samples were then incubated on ice for $15 \mathrm{~min}$ and the cells were mechanically disrupted using a $2 \mathrm{ml}$ Dounce homogenizer. Next, the lysates were centrifuged at $2000 \mathrm{rpm}$ for $5 \mathrm{~min}$ at $4^{\circ} \mathrm{C}$ and the supernatant (also: postnuclear supernatant, PNS) was collected and kept for further use. The pellet was washed $3 \mathrm{x}$ with buffer $\mathrm{A}+$ and resuspended with $50 \mu \mathrm{l}$ of Buffer $\mathrm{C}$ followed by rotating for $15 \mathrm{~min}$ at $4^{\circ} \mathrm{C}$. Subsequently, the samples were centrifuged at $14,000 \mathrm{rpm}$ for $20 \mathrm{~min}$ and the supernatant was used as nuclear fraction (NF). PNS and NF were subjected to SDS-PAGE and western blot.

\subsubsection{Centrosomal purification}

One hour before the lysis, CGNs were treated with cytochalasin D $(1 \mu \mathrm{g} / \mathrm{ml})$ and nocodazole $(0.2 \mu \mathrm{M})$. After the treatment, neurons were lysed in the NP-40 lysis buffer supplemented freshly with protease inhibitors $(1 \mu \mathrm{g} / \mathrm{ml}$ pepstatin, $1 \mu \mathrm{g} / \mathrm{ml}$ aprotinin, 1 $\mu \mathrm{g} / \mathrm{ml}$ leupeptin and $1 \mathrm{mM}$ PMSF). Next, the lysates were centrifuged with $60 \%$ sucrose at $10000 \mathrm{~g}$ for $30 \mathrm{~min}$ at $4^{\circ} \mathrm{C}$, the bottom fraction containing concentrated centrosomes was loaded onto a discontinuous sucrose gradient: $40 \%, 50 \%$ and $70 \%$, and centrifuged at $34000 \mathrm{~g}$ for $1 \mathrm{~h}$ at $4^{\circ} \mathrm{C}$. FBXO41 was immunoprecipitated from the collected fractions and used for SDS-PAGE and western blot.

\subsection{In vivo electroporation, immunohistochemistry and data analysis}

In vivo electroporation was performed on $\mathrm{P} 4 \mathrm{Wistar}$ rats as previously described (Konishi et al., 2004). $16 \mu \mathrm{g}$ of plasmid DNA and $4 \mu \mathrm{g}$ of Bclxl in a volume of $3 \mu \mathrm{l}$, together with $0.03 \%$ Fast Green, were injected per cerebellum with a 30 gauge needle. Next, the animals were subjected to 4 electrical pulses $(160-170 \mathrm{~V}$ for $50 \mathrm{~ms}, 950 \mathrm{~ms}$ interval) and 
returned to the dam. At $\mathrm{P}$ 9, the animals were sacrificed and isolated cerebella were immersed in $4 \%$ PFA diluted with PBS and incubated at $4^{\circ} \mathrm{C}$ with gentle rocking. The next day the cerebella were washed twice with PBS and $30 \%$ sucrose solution in PBS was added. After the cerebella sank to the bottom of the tubes, they were mounted into plastic molds using OCT (Tissue Tek, Sakura, Torrance, CA) and 30\% sucrose in PBS, both mixed in equal proportions. The mounting solution was allowed to solidify on dry ice and the cerebella were stored at $-80^{\circ} \mathrm{C}$. For further analysis, cerebella were cut into $40 \mu \mathrm{m}$ thin sections and the mounting medium was removed by washing with PBS for 10 min. Next, the section were incubated for 30 min with a blocking buffer (10\% normal goat serum, 2\% BSA, 0.4\% Triton X-100 in PBS). Subsequently, the mouse GFP antibody diluted in $2 \%$ BSA in PBS and $0.4 \%$ Triton X-100 was applied overnight at $4{ }^{\circ} \mathrm{C}$. Following 2x PBS wash, slides were incubated with the secondary antibody (anti-mouse Alexa 488 diluted in $2 \%$ BSA, $0.4 \%$ Triton X-100 in PBS) for $2 \mathrm{~h}$. Slides were washed twice with PBS prior to incubation with Hoechst 33258 for 5 min. Mowiol mounting medium was used for mounting a glass cover slip on a slide. Pictures of cerebellar sections were acquired with a fluorescent microscope Eclipse Ti (Nikon) or a confocal SP2 (Leica) microscope in a blinded manner. Analysis of localization of CGNs in a particular cerebellar layer or measurement of migrated distance was performed with the Fiji software (NIH).

\subsection{Generation of an FBXO41 knockout mouse}

The FBXO41 knockout ES cells were from a C57/BL6 mouse and were generated by the KOMP repository. In these cells, the whole FBXO41 gene was replaced by a $\beta$-galactosidase cassette and a neomycin resistance gene under the human ubiquitin $\mathrm{C}$ promoter. The ES cells were then injected to a blastocyst from a C57/BL6 mouse. Chimeric animal obtained after injection of the blastocyst to a foster mother, was crossed with a wild type animal and a germline transmission was achieved. Genotyping of the mouse line was performed as follows: 


\begin{tabular}{|c|c|}
\hline \multicolumn{2}{|r|}{ Genotyping PCR setup } \\
\hline Volume & Reagent \\
\hline $1 \mu \mathrm{l}$ & forward primer $(10 \mathrm{pmol} / \mu \mathrm{l})$ \\
\hline $1 \mu \mathrm{l}$ & reverse primer $(10 \mathrm{pmol} / \mu \mathrm{l})$ \\
\hline $5 \mu \mathrm{l}$ & 5 GoTaq $^{\circledR}$ buffer \\
\hline $2.1 \mu \mathrm{l}$ & dNTPs (2.5 mM) \\
\hline $0.1 \mu \mathrm{l}$ & GoTaq $^{\circledR}$ DNA polymerase \\
\hline $1 \mu \mathrm{l}$ & DMSO \\
\hline $9.3 \mu \mathrm{l}$ & $\mathrm{H}_{2} \mathrm{O}$ \\
\hline $0.5 \mu 1$ & DNA \\
\hline
\end{tabular}

\begin{tabular}{|c|c|c|}
\hline \multicolumn{3}{|c|}{ Genotyping PCR conditions } \\
\hline Temperature & Time & \\
\hline $95^{\circ} \mathrm{C}$ & $5 \mathrm{~min}$ & \\
\hline $95^{\circ} \mathrm{C}$ & $30 \mathrm{~s}$ & \\
\hline $55^{\circ} \mathrm{C}$ & $30 \mathrm{~s}$ & 18 cycles \\
\hline $68^{\circ} \mathrm{C}$ & $1 \mathrm{~min}$ & \\
\hline $72^{\circ} \mathrm{C}$ & $10 \mathrm{~min}$ & \\
\hline $16^{\circ} \mathrm{C}$ & $\infty$ & \\
\hline
\end{tabular}

Primers listed below were used for the genotyping PCR:

Forward WT/KO: 5’ ATCCACCTAGACCTAGTAATCTTA 3'

Reverse WT: 5' CTTCTCCAGGGCGCGGAT 3'

Reverse KO: 5’ AATGCGCTCAGGTCAAATTCAG 3 


\subsection{Generation of the FBXO41 antibody}

The rabbit polyclonal FBXO41 antibody was generated by Eurogentec. The antigen was a short fragment of mouse FBXO41 expressed in BL21 E. coli and affinity-purified using Ni-NTA Sepharose (Qiagen), since the antigen harbored a polyhistidine tag. 


\section{Results}

\subsection{Characterization of FBXO41 expression}

\subsubsection{FBXO41 is a CNS-specific neuronal protein}

Growing evidence demonstrates the importance of F-box proteins in neurodevelopment and pathogenesis of neurological disorders (Westbrook et al., 2008, Zhao et al., 2011, Hoeck et al., 2010, Wu et al., 2013, Boix-Perales et al., 2007, Jandke et al., 2011, Litterman et al., 2011, Saiga et al., 2009, Fonzo et al., 2009, Gong et al., 2010, Liao et al., 2004). Owing to this fact, the aim of my study was to characterize novel F-box proteins essential for brain development. A search of the Cerebellar Development Transcriptome Database (CDT-DB) led to the brain-specific F-box protein FBXO41, whose specific expression in the cortex, hippocampus and cerebellum was confirmed by RT-PCR (Fig. 3.1 A).

In order to examine FBXO41 expression at the protein level, I first verified the specificity of the custom made FBXO41 antibody by means of antigen competition. I used tissue lysates from cortex and spleen, the latter serving as a negative control, and subjected them to immunoblotting either with the untreated FBXO41 antibody or with the FBXO41 antibody, which was exposed to the antigen prior to incubation with the nitrocellulose membrane. While the antibody on its own revealed a band of approximately $100 \mathrm{kDa}$, the antibody preincubated with the antigen failed to do so (Fig. 3.1 B). Using this specific FBXO41 antibody, I demonstrated that FBXO41 was expressed in the mouse brain (cortex, cerebellum and hippocampus), but not in the lung, liver, heart, kidney, spleen and testis (Fig. 3.1 C). Additionally, C. Mukherjee found that FBXO41 was expressed in the spinal cord but not in the sciatic nerve. These finding revealed 

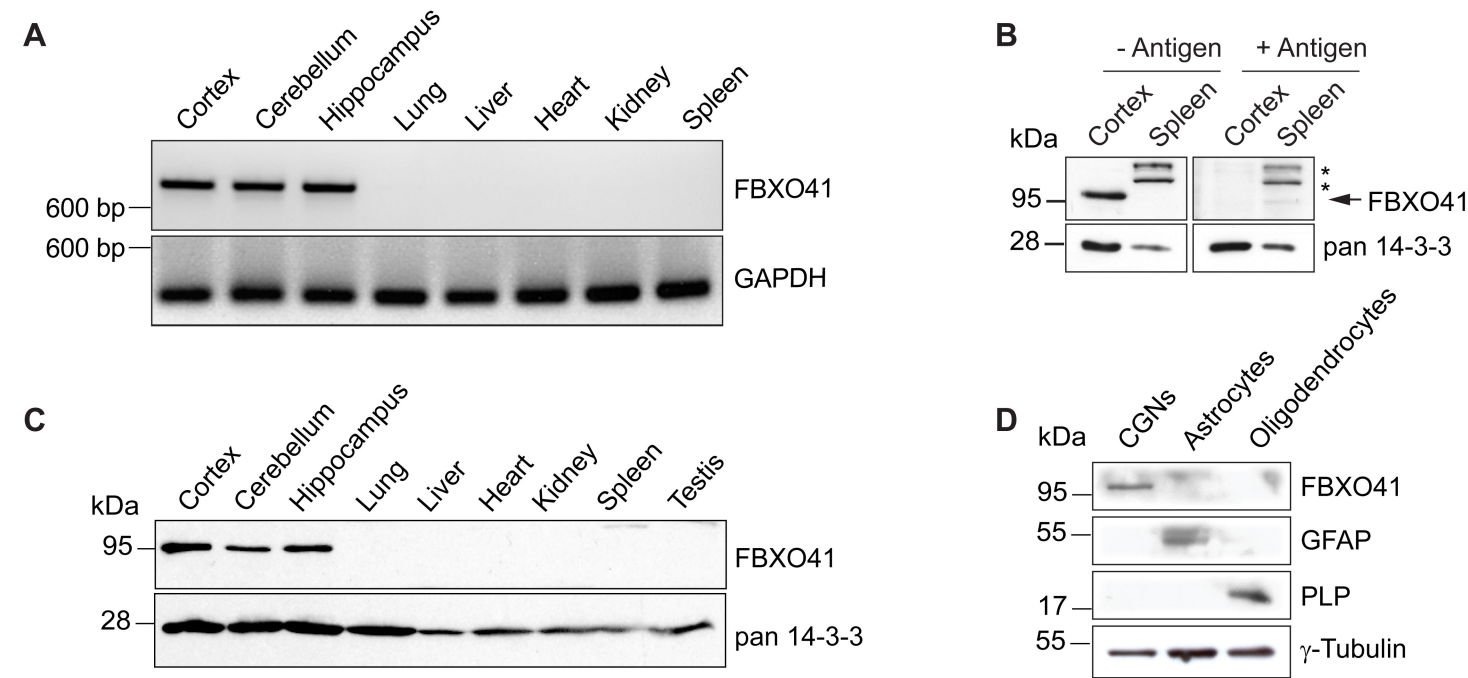

Figure 3.1.: FBXO41 is a brain-specific neuronal protein. (A) FBXO41 mRNA was isolated from a P20 mouse and cDNA was synthesized. RT-PCR was performed with primers located in FBXO41 and GAPDH cDNA. The latter was used as an internal control. The experiment was conducted by Dr. R. Busse. (B) Lysates from mouse cortex and spleen were immunoblotted with the FBXO41 antibody alone or with with the FBXO41 antibody preabsorbed with the antigen. Pan 14-3-3 was used as a loading control. Asterisks indicate non-specific bands. (C) Indicated tissues from a P20 mouse were lysed and subjected to immunoblotting with the FBXO41 antibody and the pan 14-3-3 antibody served as a loading control. (D) Lysates from cultured CGNs, astrocytes and oligodendrocytes were probed with the FBXO41 antibody as well as the GFAP and PLP antibodies, which served as positive controls for astrocytes and oligodendrocytes, respectively. $\gamma$-Tubulin antibody was used as a loading control.

that FBXO41 is a central nervous system-specific protein.

Having established that FBXO41 is exclusively expressed in the brain, I went on to investigate the expression of FBXO41 in different cell types present in the CNS. For this purpose, I prepared total lysates from cultured cerebellar granule neurons (CGNs), astrocytes and oligodendrocytes, which I subsequently subjected to immunoblotting with the FBXO41 antibody (Fig. 3.1 D). I found that only neurons but neither astrocytes, nor oligodendrocytes express FBXO41. The analysis supports the notion that FBXO41 is a neuron-specific protein. 


\subsubsection{FBXO41 is expressed in embryonic and adult brain tissues}

To answer the question if FBXO41 may play an important role in brain development, it was essential to characterize its temporal expression pattern. For this purpose, I isolated heads from E10, total brain from E12 or cortices from E14, 16 and 18 mouse embryos and subjected the lysates to immunoblotting with the FBXO41 antibody. I found that FBXO41 was already detectable in the E10 embryo and its expression increased during the embryonic development (Fig. 3.2 A). Moreover, I examined FBXO41 expression in the cortex, cerebellum and hippocampus of older mice (E18 to P60). These data show that FBXO41 was expressed at all time points analyzed and its amount increased with the age in the cerebellum and hippocampus (Fig. 3.2 B).

A

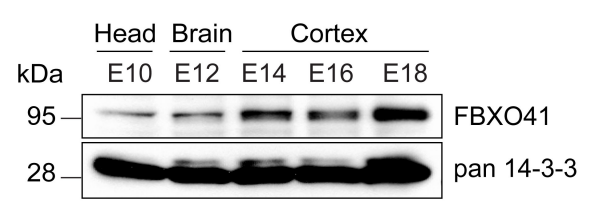

B kDa E18 P0 P3 P6 P9 P14 P30 P60

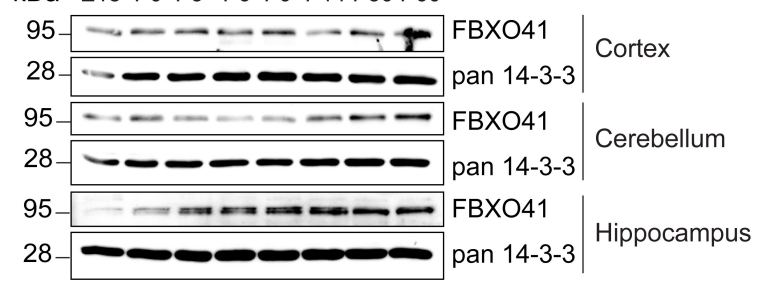

Figure 3.2.: FBXO41 is expressed from embryonic stages to adulthood. (A, B) Lysates from indicated tissues were isolated from E10-P60 mice and immunoblotted with the FBXO41 or the pan 14-3-3 antibodies. The latter served as a loading control.

\subsubsection{FBXO41 is expressed in cultured neurons}

Since cultured neurons are an excellent model system to investigate protein function in the brain, I determined the expression of FBXO41 in cultured CGNs in addition to cortical and hippocampal neurons. In order to do so, I harvested these neurons at different days in vitro (DIV). Subsequent immunoblotting of the lysates with the FBXO41 antibody revealed that FBXO41 was present in all neuronal types and its expression peaked around DIV 6 (Fig. 3.3), demonstrating that cultured neurons express FBXO41. 


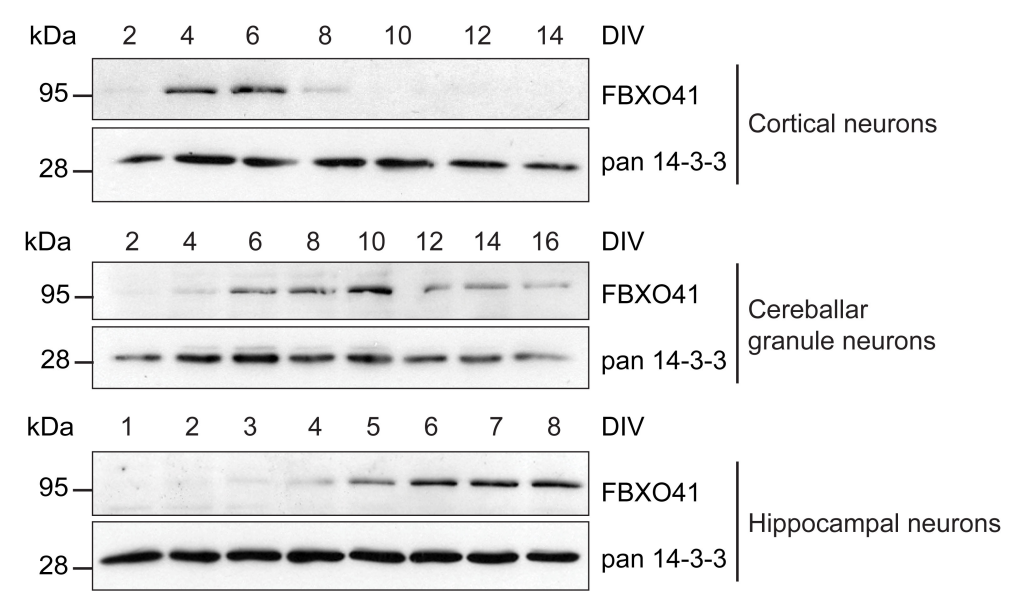

Figure 3.3.: Cultured neurons express FBXO41. Lysates from cortical and hippocampal neurons as well as from CGNs were prepared at indicated DIVs and probed with the FBXO41 antibody. Pan 14-3-3 antibody was used as a loading control.

\subsubsection{FBXO41 is localized to the centrosome and cytoplasm of neurons}

To dissect the expression of FBXO41 at the subcellular level, I transfected cultured CGNs with a plasmid encoding GFP-FBXO41 and subjected the neurons to immunostaining with the GFP antibody. I found that FBXO41 was localized to the cytoplasm, including axons and dendrites, and to the centrosome. Furthermore, FBXO41 was absent from the nucleus (Fig. 3.4 A). Further subcellular fractionation and centrosomal purification analyses of CGNs verified FBXO41's cytoplasmic localization and presence at the centrosome, respectively (Fig. 3.4 $\mathrm{B}$ and C). Taken together, these results revealed that FBXO41 is a cytoplasmic and centrosomal protein. 
A
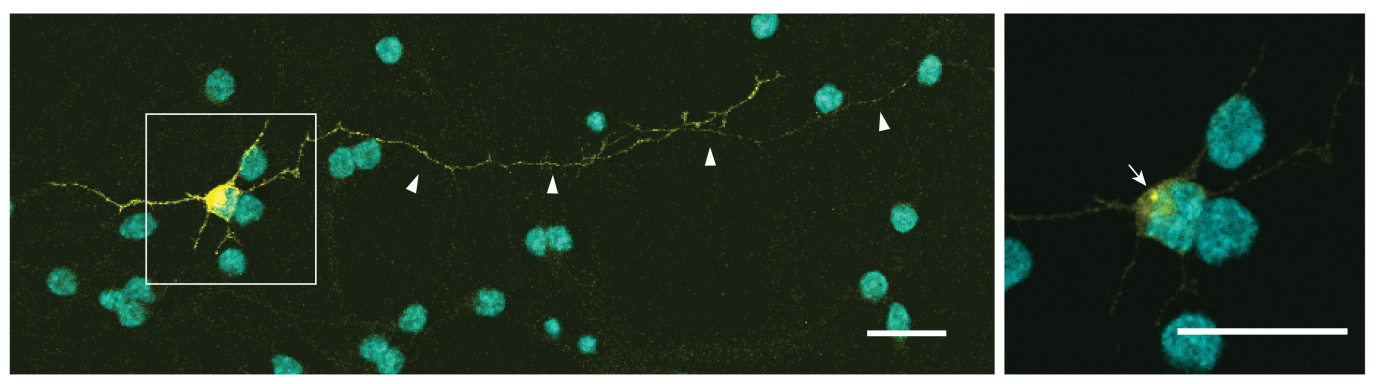

B
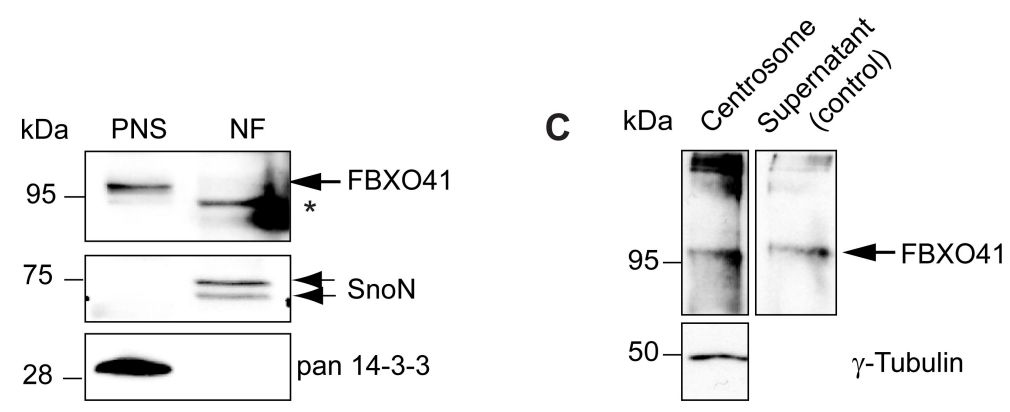

Figure 3.4.: FBXO41 localizes to the centrosome and cytoplasm of cerebellar granule neurons. (A) CGNs were transfected at DIV 2 with GFP-FBXO41 and subjected to immunostaining using the GFP antibody and the DNA dye 4',6-diamidino-2-phenylindole (DAPI) 1.5 days later. Arrowheads indicate axon, arrow indicates centrosomal FBXO41. Scale bar: $20 \mu \mathrm{m}$. The image was taken by Dr. M. Vadhvani. (B) Subcellular fractionation was performed using CGNs prior to immunoblotting with the FBXO41 antibody. Pan 14-3-3 and SnoN antibodies were used as controls for the postnuclear supernatant (PNS) and the nuclear fraction (NF), respectively. Asterisk indicates a non-specific band. This analysis was performed by Dr. M. Kannan. (C) CGNs were subjected to centrosomal purification followed by immunoprecipitation with the FBXO41 antibody. Immunoprecipitates were probed with the FBXO41 antibody. $\gamma$-Tubulin served as a positive control for the centrosomal fraction. The experiment was conducted by $\mathrm{N}$. Schwedhelm-Domeyer.

\subsection{Functional analysis of FBXO41 in the neural development}

Based on FBXO41 expression in the developing brain and its centrosomal localization, we reasoned that FBXO41 may play an essential role in neurodevelopmental processes 
like neuronal morphogenesis, migration and survival. In this study, I used gain- and loss-of-function approaches to elucidate the function of FBXO41 in axon and dendrite growth, establishment of neuronal polarity, migration and survival.

\subsubsection{The role of FBXO41 in neuronal morphogenesis}

\subsubsection{Knockdown of FBXO41 in CGNs inhibits axon growth}

To examine the role of FBXO41 in neuronal morphology, I took an RNAi approach, for which I first tested the efficacy of a set of FBXO41 RNAi plasmids generated in the lab (Fig. 3.5 A). Here, I transfected HEK 293T cells with the myc-FBXO41 plasmid together with different FBXO41 RNAi plasmids and subjected lysates, obtained 4 days later, to immunoblotting. The experiment indicated that FBXO41 RNAi\#5 efficiently downregulated FBXO41 expression (Fig. 3.5 B).

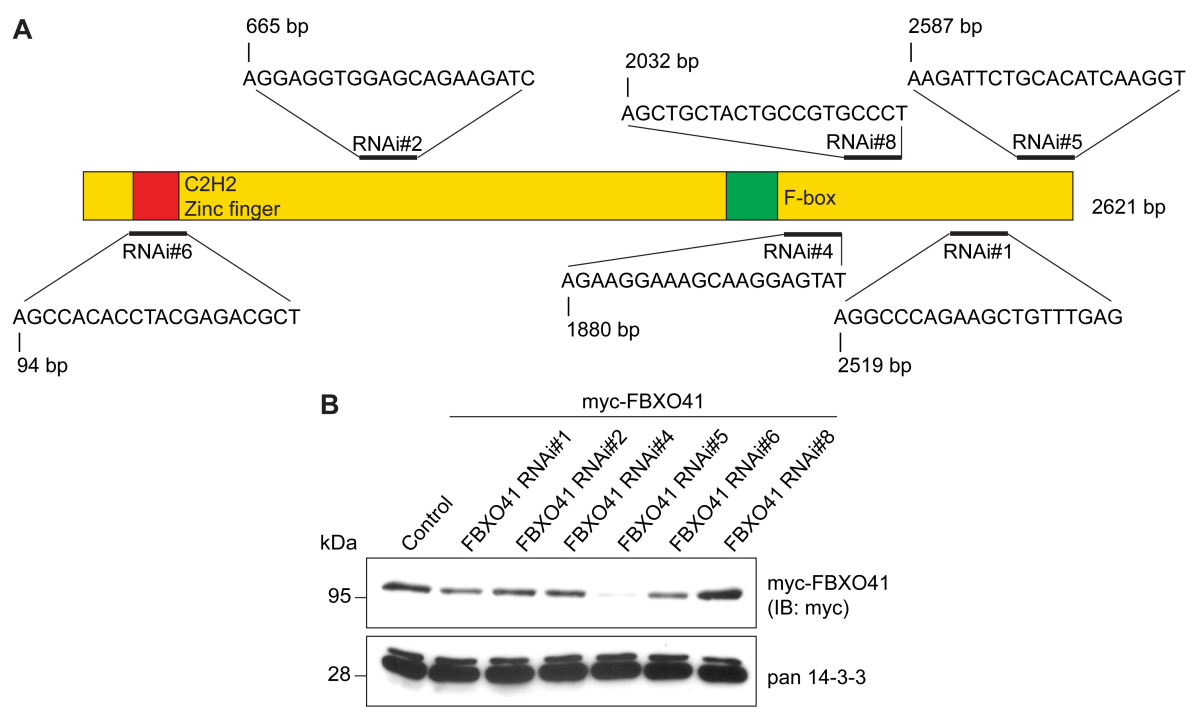

Figure 3.5.: RNAi\#5 efficiently knocks down FBXO41. (A) Schematic of FBXO41 with indicated domains as well as RNAi targeting regions. (B) HEK 293T cells were transfected with myc-FBXO41 and U6 empty control vector or RNAi plasmids: \#1, \#2, \#4, \#5, $\# 6$ and \#8. After 4 days, cell lysates were immunoblotted with the myc antibody. Pan 14-3-3 served as a loading control. 
In the next step, I transfected cultured CGNs at DIV 0 with the empty control plasmid, the functional FBXO41 RNAi\#5 or the non-functional FBXO41 RNAi\#2 and analyzed neuronal morphology 4 days later. I found that FBXO41 knockdown resulted in significantly shorter axons as compared to the control condition (Fig. 3.6). Expression of the non-functional FBXO41 RNAi in turn had little or no effect on axon growth (Fig. 3.6). This experiment indicates that FBXO41 promotes axon growth in cerebellar granule neurons.
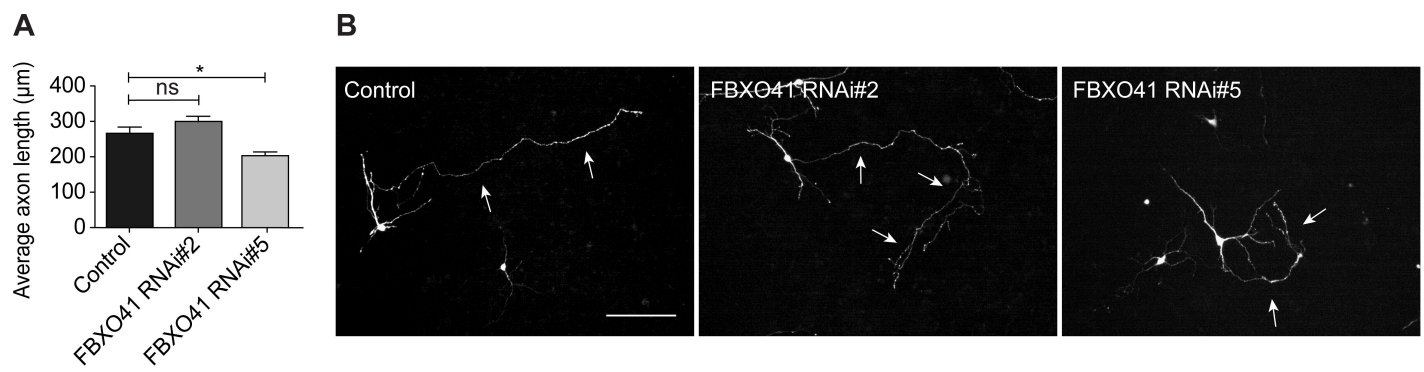

Figure 3.6.: FBXO41 RNAi inhibits axon growth. (A) CGNs transfected at DIV0 with empty U6 vector, FBXO41 RNAi\#2 (non-functional) or \#5 (functional) and a GFPencoding plasmid were immunostained with the GFP antibody 4 days later, followed by morphological analysis. A total of 431 neurons were measured (ANOVA, ${ }^{*} p<0.05$, ns $=$ non-significant, mean + s.e.m.). (B) Representative images of (A). Arrows indicate axons. Scale bar: $100 \mu \mathrm{m}$.

To exclude possible off-target effects of FBXO41 RNAi, I conducted a rescue experiment, for which I generated an FBXO41-Res mutant by introducing 4 silent mutations into the RNAi\#5 targeting region of FBXO41, with site-directed mutagenesis (Fig. 3.7 A). Then I co-transfected HEK $293 \mathrm{~T}$ cells with the empty U6 vector together with the myc-FBXO41 WT as a control condition and the FBXO41 RNAi\#5 together with either empty myc vector or FBXO41-Res expression plasmid. Four days later, I analyzed the lysates using immunoblotting and observed that the levels of the FBXO41-Res but not of the wild type FBXO41 were restored when RNAi\#5 was co-expressed. This result demonstrates that the FBXO41-Res plasmid is resistant to RNAi-induced knockdown (Fig. 3.7 B). 

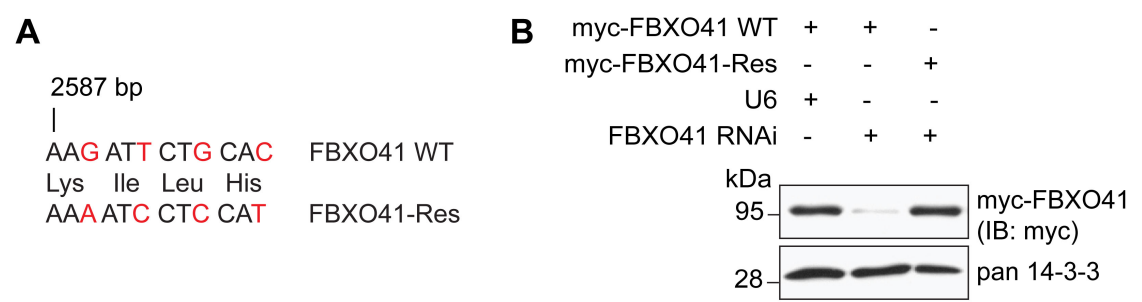

Figure 3.7.: FBXO41-Res is resistant to RNAi-induced knockdown. (A) Silent mutations in the targeting region of FBXO41 are indicated in red. (B) HEK293T cells were transfected with indicated plasmids and lysed 4 days later. Lysates were immunoblotted with the myc antibody and the pan 14-3-3 antibody for loading control.

I subsequently transfected CGNs at DIV 0 with the empty control U6 and myc vectors or with the FBXO41 RNAi\#5 together with either the empty myc vector or mycFBXO41-Res and fixed the cells 4 days later. Morphometric analysis revealed that the axon length is reduced upon the FBXO41 knockdown, but can be restored to baseline of control condition by overexpressing the RNAi-resistant mutant of FBXO41 (Fig. 3.8). The experiment established FBXO41 as a regulator of axon growth in CGNs.
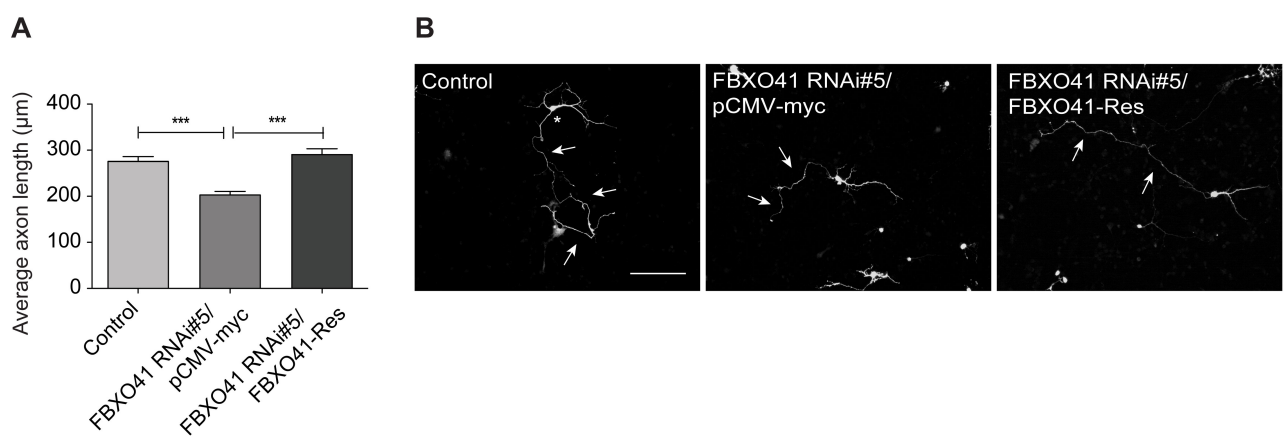

Figure 3.8.: FBXO41-Res overexpression restores axon growth in FBXO41 knockdown CGNs. (A) CGNs transfected at DIV0 with empty U6 and pCMV-myc vectors (control) or FBXO41 RNAi\#5 together with either pCMV-myc or FBXO41-Res were morphometrically analyzed at DIV 4. 621 neurons were measured (ANOVA, ${ }^{* * *} \mathrm{p}<0.001$, mean + s.e.m.). (B) Representative images of (A). Arrows indicate axons. Scale bar: $100 \mu \mathrm{m}$. 


\subsubsection{Axon growth is reduced in CGNs of FBXO41 knockout mice}

To study the role of FBXO41 in brain development in vivo, we generated an FBXO41 knockout $(\mathrm{KO})$ mouse, in which the entire FBXO41 gene was deleted and replaced by a $\beta$-galactosidase reporter cassette and neomycin selection cassette (Fig. 3.9 A). For the genotyping PCR, specific sets of primers were designed to detect the wild type and the mutant allele (Fig. 3.9 B).

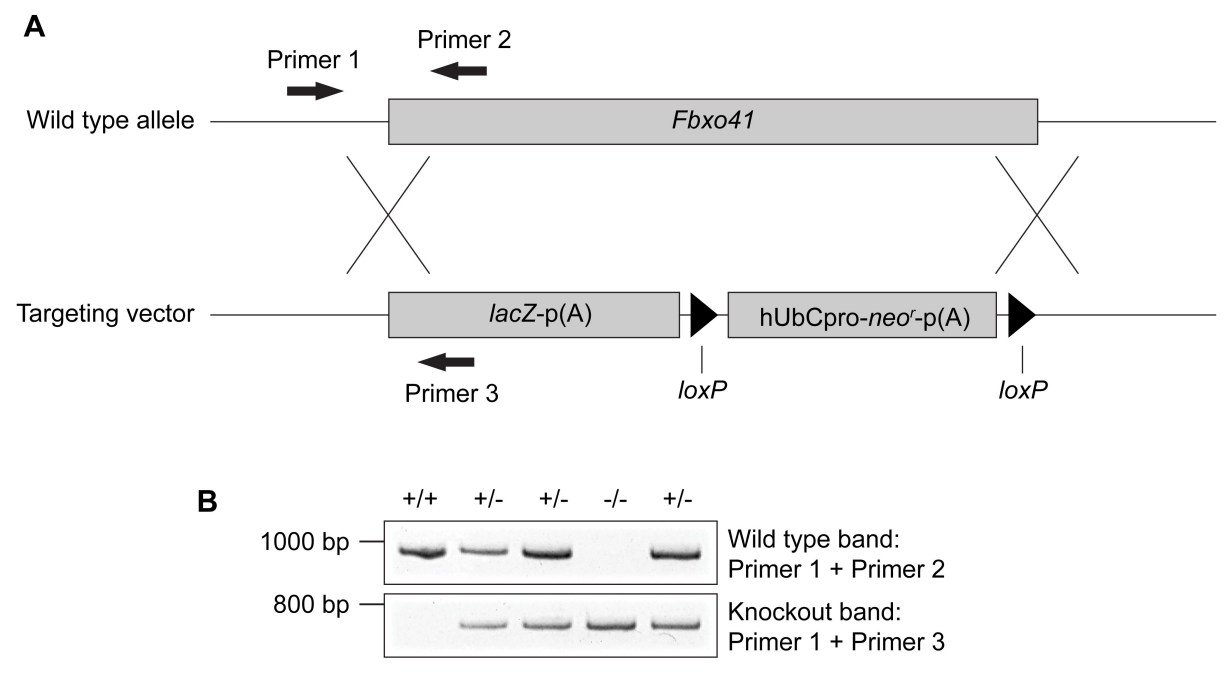

Figure 3.9.: FBXO41 KO mouse generation strategy. (A) In ES cells purchased from KOMP, the entire FBXO41 gene was replaced by a $\beta$-galactosidase encoding sequence and a neomycin resistance gene under the human ubiquitin $\mathrm{C}$ promoter. (B) Representative genotyping PCR of mice following germline transmission. Location of the primers is indicated in $(\mathrm{A})$.

Upon proper germline transmission, I verified the deletion of FBXO41 by subjecting lysates from P5 and P16 FBXO41 ${ }^{+/+}, \mathrm{FBXO} 41^{+/-}$and FBXO41-/- cortex, cerebellum and hippocampus to immunoblotting with the FBXO41 and the $\beta$-Gal antibody. FBXO41 is completely absent from the $\mathrm{KO}$ mice. In heterozygous FBXO41 $\mathrm{KO}$ brains, I found half of the amount as compared to wild type mice. Conversely, while $\beta$-Gal was absent from wild type brain, I detected increasing amounts in heterozygous and homozygous brains (Fig. 3.10). In addition, the $\beta$-Gal expression pattern goes in line with the previous 
observations that FBXO41 is present in the cortex, cerebellum and hippocampus. This finding confirms the efficient ablation of FBXO41 and a successful integration of the reporter cassette.
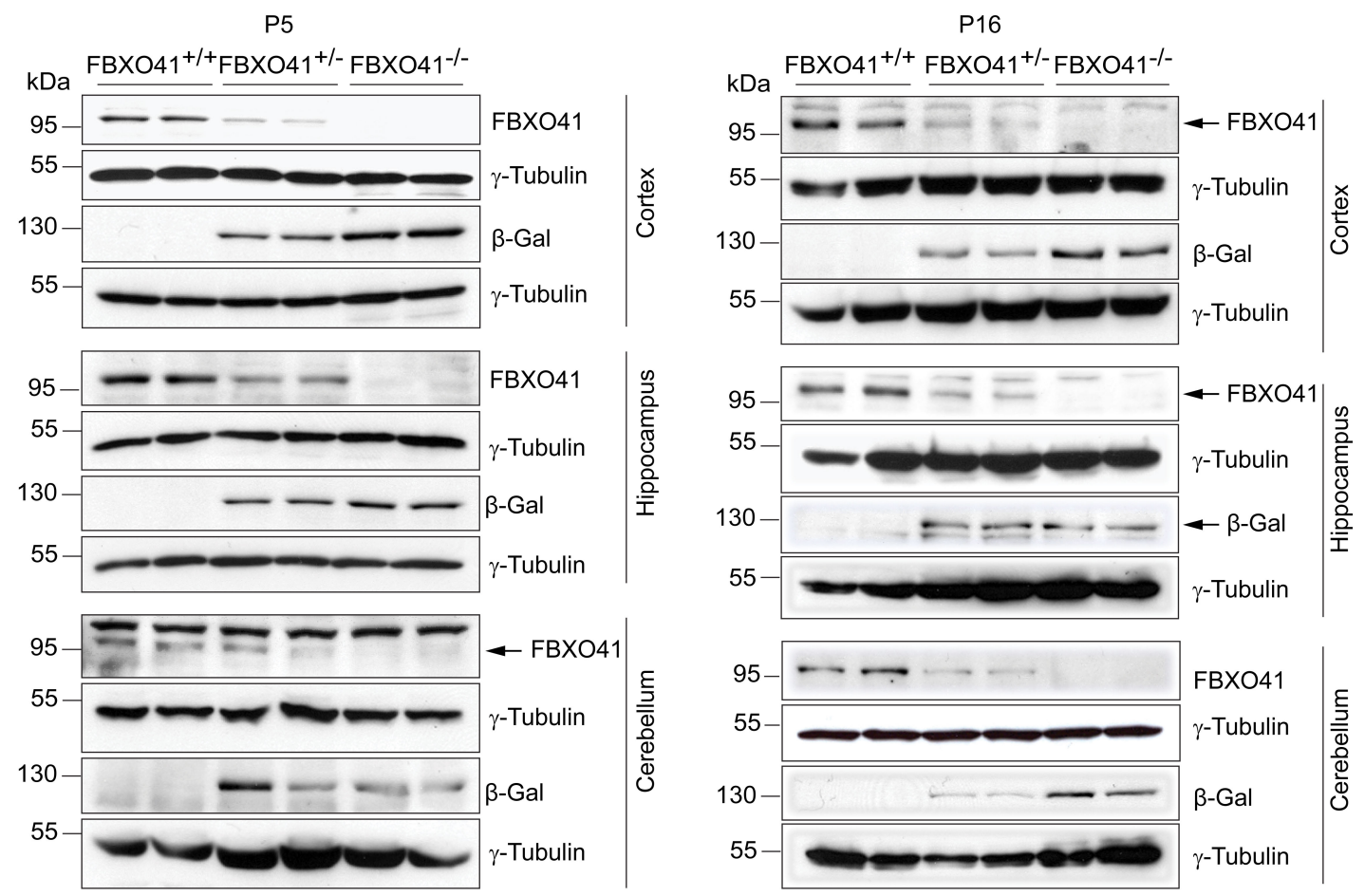

Figure 3.10.: Successful deletion of FBXO41 in the brain. Lysates from cortex, hippocampus and cerebellum of P5 and P16 mice were immunoblotted with the FBXO41 and the $\beta$-Gal antibody. $\gamma$-Tubulin served as a loading control.

Having established the levels of FBXO41 in wild type, heterozygous and homozygous brains, I analyzed axonal growth in cultured cerebellar granule neurons isolated from P5 pups of the aforementioned genotypes. At DIV 2, I transfected the neurons with the GFP expression plasmid to visualize their morphology. Six days later, I measured the axonal length. I discovered that axons of FBXO41-/- CGNs were significantly shorter than the axons of the wild type neurons. Furthermore, axons of the heterozygous CGNs had an intermediate length (Fig. 3.11). These results indicate that FBXO41 stimulates axon growth. 

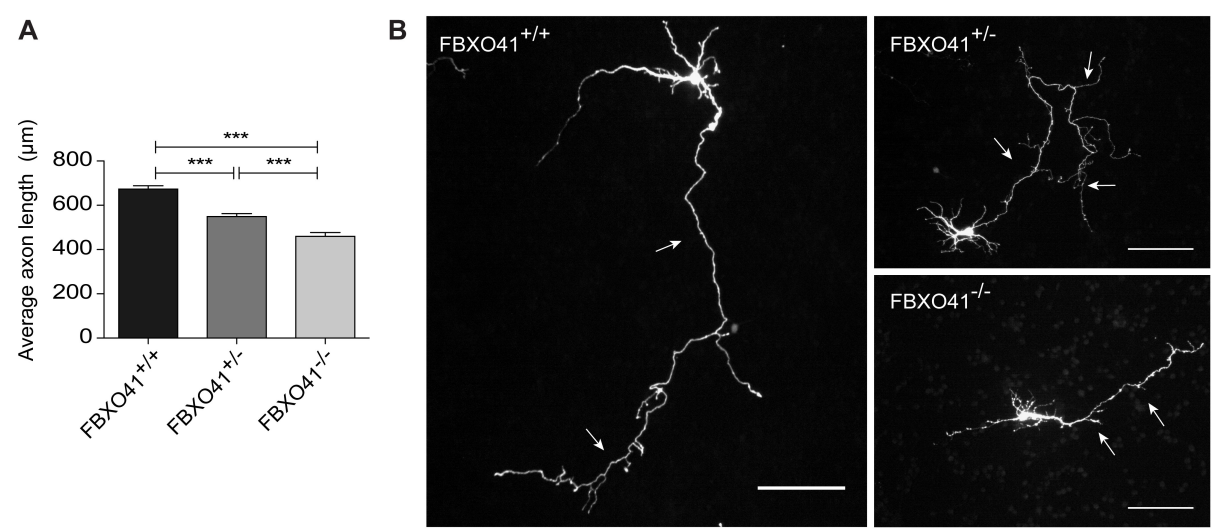

Figure 3.11.: Axon growth is reduced in the CGNs from FBXO41 knockout mouse. (A) CGNs from the FBXO41 ${ }^{+/+}, \mathrm{FBXO} 41^{+/-}$and $\mathrm{FBXO} 41^{-/-}$mice were transfected with a GFP-encoding plasmid at DIV 2 and immunostained with the GFP antibody at DIV 8. Axons of 908 neurons were measured (ANOVA, ${ }^{* * *} \mathrm{p}<0.001$, mean + s.e.m.). (B) Representative images of (A). Arrows indicate axons. Scale bar: $100 \mu \mathrm{m}$.

Further, I performed a rescue experiment, in which I expressed FBXO41 in FBXO41 knockout CGNs and compared the axon length with the FBXO41 wild type and knockout neurons, both transfected with the empty myc plasmid (Fig. 3.12) .
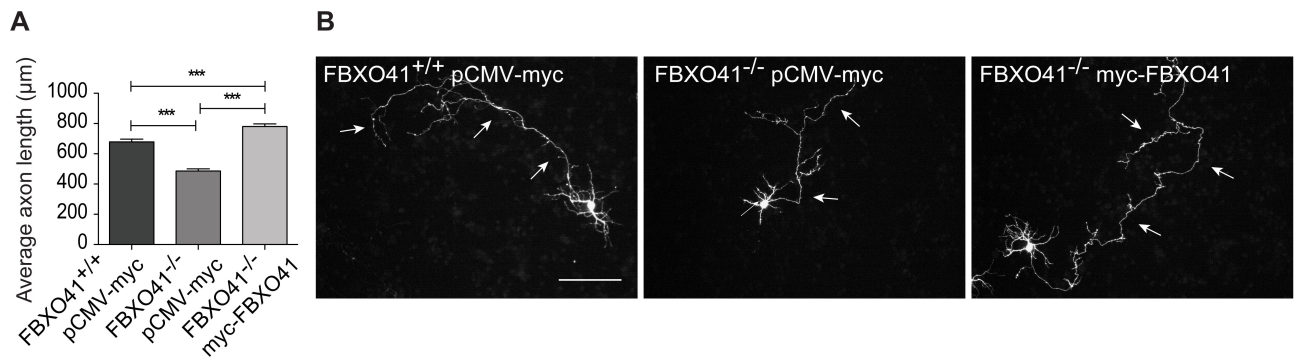

Figure 3.12.: Overexpression of FBXO41 rescues axon growth in the FBXO41-/neurons. (A) FBXO41 $1+$ CGNs were transfected with the pCMV-myc vector and FBXO41-/- CGNs with the pCMV-myc vector or myc-FBXO41 together with a GFP plasmid. Axon length was measured at DIV8. A total of 693 neurons was measured (ANOVA, ${ }^{* * *} \mathrm{p}<0.001$, mean + s.e.m.). (B) Representative images of (A). Arrows indicate axons. Scale bar: $100 \mu \mathrm{m}$. 
Consistent with previous results, axon length was reduced in the KO CGNs but axon growth was restored in the KO neurons overexpressing FBXO41. Thus, the axon growth impairment is due to the loss of FBXO41 (Fig. 3.12).

\subsubsection{Overexpression of FBXO41 promotes axon growth in CGNs}

While the preceding experiments established a role for FBXO41 in axon growth of cerebellar granule neurons, I addressed whether FBXO41 gain-of-function would reveal the opposite phenotype. Therefore, I transfected CGNs at DIV 0 with the empty vector or the FBXO41 expression plasmid and analyzed axon length 3 days later. I observed significantly longer axons upon FBXO41 overexpression as compared to the control (Fig. 3.13). This experiment established, that gain-of-function of FBXO41 promotes axon growth of CGNs.
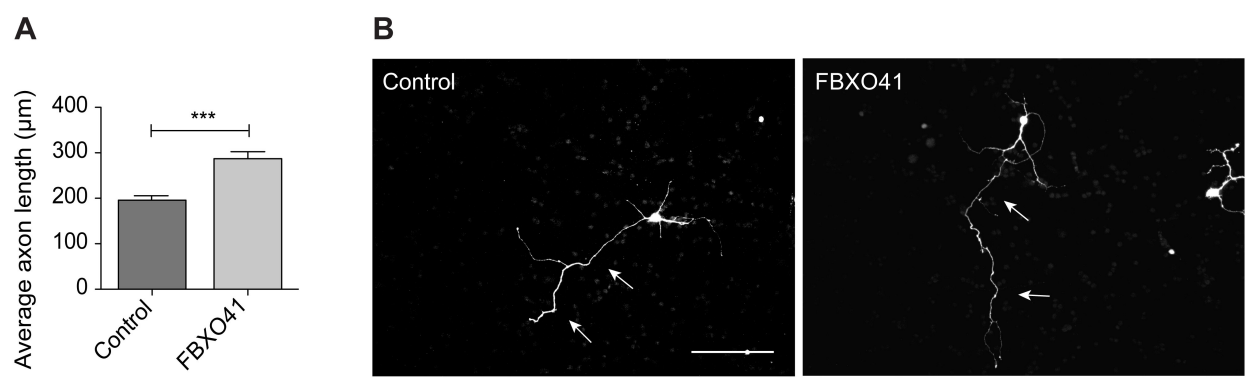

Figure 3.13.: Overexpression of FBXO41 promotes axon growth in CGNs (A) FBXO41 were transfected with the empty pCMV-myc plasmid or with myc-FBXO41 and the axon length of 372 neurons was measured 3 days later (t-test, ${ }^{* *} \mathrm{p}<0.001$, mean + s.e.m.). (B) Representative images of (A). Arrows indicate axons. Scale bar:100 $\mu \mathrm{m}$.

\subsubsection{FBXO41 controls axon growth independently of the neuronal subtype}

To investigate if the FBXO41-regulated axon growth is a generalizable mechanism, I isolated and cultured neurons from the E18 rat hippocampi and transfected them at DIV 1 with the empty control vector, the functional FBXO41 RNAi\#5 or the non-functional 
FBXO41 RNAi\#8. Morphological analyses at DIV5 revealed that the functional RNAi causes the axons to grow significantly shorter than the non-functional RNAi or the control vector (Fig. 3.14), which suggests that FBXO41 regulates axon growth in a neuronal subtype-independent manner.
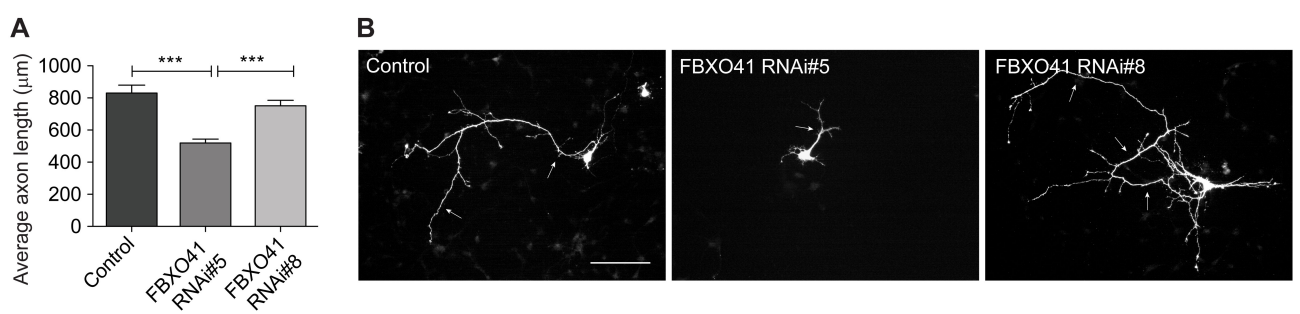

Figure 3.14.: Knockdown of FBXO41 inhibits axon growth in hippocampal neurons. (A) Hippocampal neurons were transfected at DIV1 with the control U6 vector or the FBXO41 RNAi \#5 and \#8. Neurons were fixed 4 days later and the axon length was measured. A total of 371 neurons was analyzed (ANOVA, ${ }^{* * *} \mathrm{p}<0.001$, mean + s.e.m.). (B) Representative images of (A). Arrows indicate axons. Scale bar: $100 \mu \mathrm{m}$.

\subsubsection{FBXO41-deficient neurons display proper dendrite growth and axon-dendrite polarity}

Having determined the role of FBXO41 in axon growth, I analyzed if it also plays a role in dendrite growth and the establishment of neuronal polarity. First, I examined the effect of FBXO41 loss-of-function on dendrite growth of CGNs transfected with the empty U6 vector or the FBXO41 RNAi\#5 at DIV 0. Four days later, I measured total dendrite length and did not observe any significant difference between the knockdown and the control neurons (Fig $3.15 \mathrm{~A}$ ).

To further corroborate this result, I assessed the complexity of dendrites from cultured FBXO41 $1^{+/+}$and FBXO41-/- cerebellar granule neurons using Sholl analysis. This experiment also did not show any difference in the overall complexity of the wild type as compared to knockout neurons (Fig. 3.15 B). These results indicate that FBXO41 does not control dendrite growth or branching.

Cultured hippocampal neurons represent a well-characterized system to study neu- 

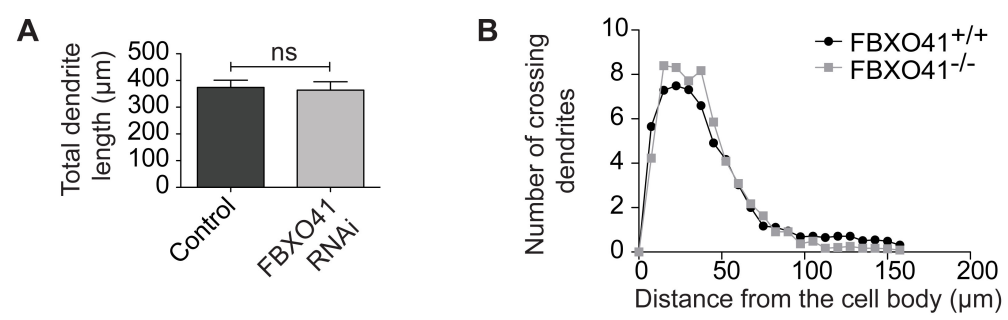

Figure 3.15.: FBXO41 does not affect dendrite growth or complexity (A) CGNs were transfected at DIV0 with the U6 control vector or the FBXO41 RNAi plasmid and analyzed 4 days later. Total dendritic length of 352 neurons was measured (t-test, ns = non-significant, mean + s.e.m.). (B) $\mathrm{FBXO} 41^{+/+}$and $\mathrm{FBXO} 41^{-/-}$mouse CGNs were transfected at DIV2 with a GFP plasmid and subjected to immunostaining with the GFP antibody 6 days later. Dendrite complexity was assessed with the Sholl analysis using ImageJ software. 70 neurons were analyzed.

ronal polarity (Bartlett et al., 1984, Dotti et al., 1988, Caceres et al., 1984, Davis et al., 1987, Bartlett and Banker, 1984). Hence, I transfected hippocampal neurons with the

A

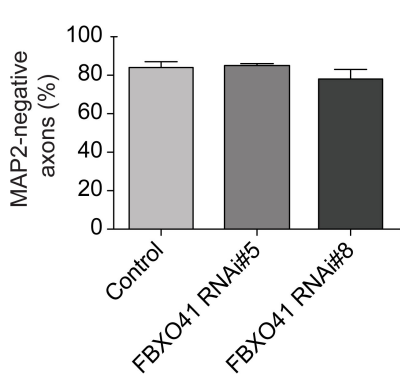

B

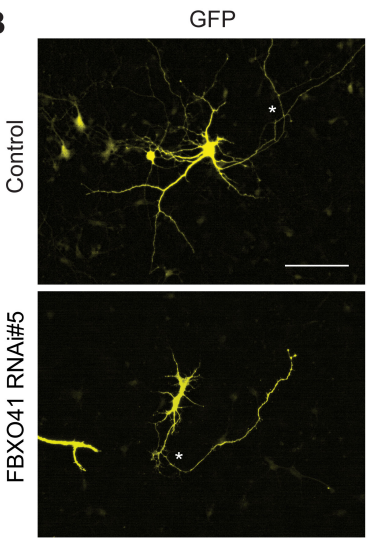

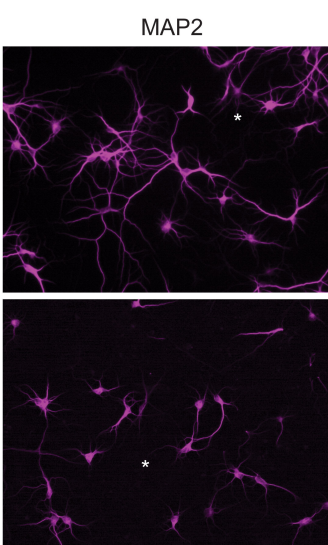

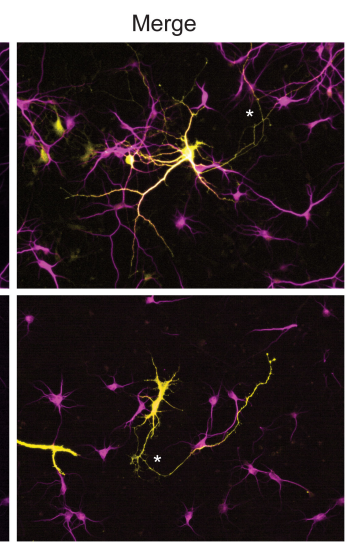

Figure 3.16.: FBXO41 is not required for establishment of neuronal polarity. (A) Hippocampal neurons, transfected at DIV1 with the U6 control vector or with a functional (\#5) or non-functional (\#8) FBXO41 RNAi together with a GFP plasmid, were subjected to immunostaining with the GFP and MAP2 antibodies 4 days later. Percentage of neurons with MAP2 expression restricted to dendrites and cell body was assessed. 364 neurons were analyzed. (B) Representative images of (A). Asterisks indicate axons. Scale bar: $100 \mu \mathrm{m}$. 
FBXO41 RNAi\#5, \#8 or with the control U6 plasmid to asses if knockdown of FBXO41 changes the distribution of the somatodendritic marker, MAP2. I observed that in all conditions, neurons exhibit MAP2 expression in the cell body and dendrites but not in the axons, indicative of proper polarization (Caceres et al., 1984), (Fig. 3.16). The experiment suggests that FBXO41 is not involved in the establishment of neuronal polarity.

\subsubsection{FBXO41 promotes neuronal migration}

During development, neurons not only undergo dramatic morphological changes, but also migrate considerable distances to reach their destinations. Since many centrosomal proteins like LIS1, DCX, DISC1 or NDEL1 have been reported to control neuronal migration (Reiner et al., 1995, Sossey-Alaoui et al., 1998, Kamiya et al., 2005, Shu et al., 2004), I was prompted to examine the role of FBXO41 in this process. To analyze FBXO41 in neuronal migration, I took advantage of the in vivo electroporation technique. Here, plasmids were injected into the cerebella of $\mathrm{P} 4$ rat pups, which were subjected to electrical pulses. After 5 days, migrating neurons of coronal cerebellar sections were analyzed. In the developing cerebellum, granule neurons migrate in a radial manner, emanating from the EGL, passing the ML and eventually settling in the IGL.

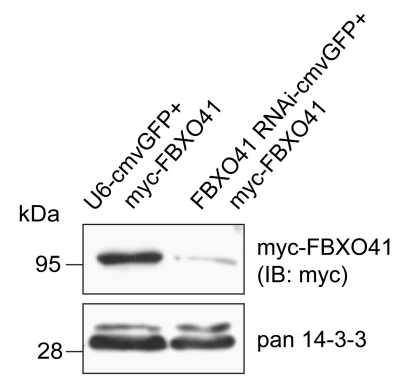

Figure 3.17.: FBXO41 RNAi bicistronic construct efficiently downregulates FBXO41. (A) HEK 293T cells were transfected with the myc-FBXO41 plasmid and with U6cmvGFP empty vector or the FBXO41 RNAi-cmvGFP plasmid and lysed after 4 days. Lysates were immunoblotted with the myc antibody. Pan 14-3-3 served as a loading control.

Strikingly, I found that FBXO41 RNAi (Fig. 3.17) reduced neuronal migration. While 
more than $90 \%$ of control neurons migrated from the EGL through the ML into the IGL, $42 \%$ of FBXO41 knockdown neurons were markedly stalled in the EGL/ML. These findings indicate that FBXO41 is required for neuronal migration.

The aforementioned finding is consistent with the results of histological analysis that my colleague, C. Mukherjee, performed on P16 cerebella of wild type and homozygous FBXO41 mice. P16 represents an age, in which the cerebellar lamination is completed. The hematoxilin and eosin staining revealed proper development of wild type cerebella, while the ML of FBXO41-/- cerebella displayed a residual EGL and neurons of elongated shape, that did not complete their migration yet. This experiment indicates, that FBXO41 is crucial for neuronal migation in vivo.
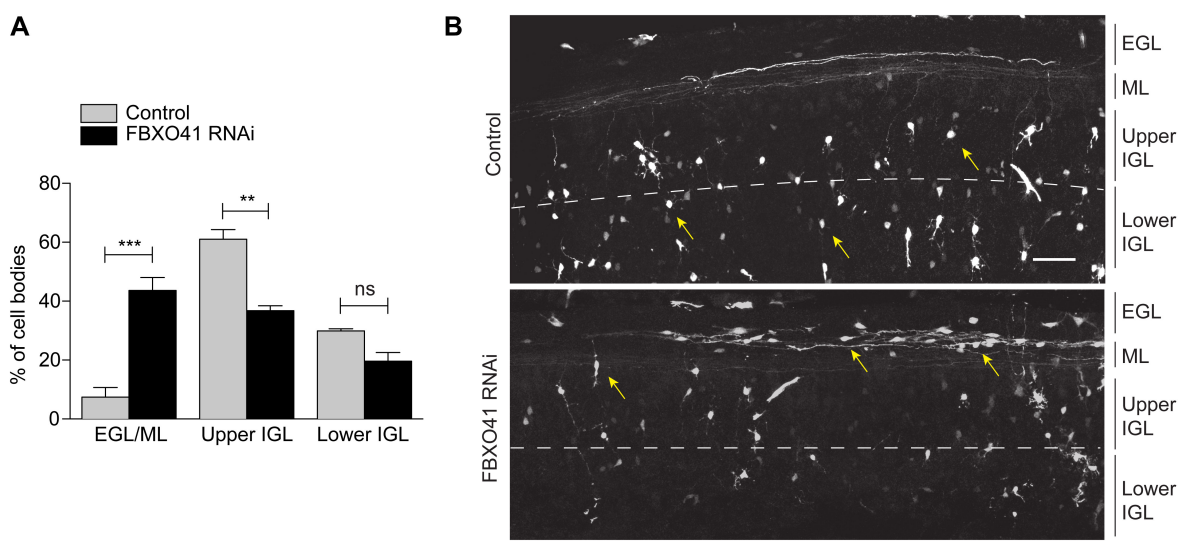

Figure 3.18.: FBXO41 regulates neuronal migration in the cerebellar cortex. (A) P4 rat cerebella were electroporated with the U6/EGFP control vector and the FBXO41 RNAi/GFP construct and isolated 5 days later. Coronal sections were immunostained with the GFP antibody and the CGN cell bodies in each layer were counted. A total of 2347 neurons was analyzed (ANOVA, ${ }^{* *} \mathrm{p}<0.01,{ }^{* * *} \mathrm{p}<0.001$, ns $=$ non-significant, mean + s.e.m.). $\mathrm{EGL}=$ external granular layer, $\mathrm{ML}=$ molecular layer, $\mathrm{IGL}=$ internal granular layer. (B) Representative images of (A). Arrows indicate cell bodies. Scale bar: $50 \mu \mathrm{m}$. The in vivo electroporation was performed by Dr. J. Stegmüller and the confocal images were taken by Dr. M. Vadhvani. 


\subsubsection{Survival of $P 5$ and $P 16$ neurons is not affected by the loss of FBXO41}

FBXO41 ${ }^{-/-}$mice exhibit high postnatal mortality and reduced body weight (Fig. 3.19) as well as severe motor defects (C. Mukherjee, unpublished data). Therefore, I used biochemical methods to investigate if levels of proteins involved in apoptotic and survival pathways are changed in these mice. First, I analyzed the expression of cleaved caspase 3 (CC3) in the FBXO41 ${ }^{+/+}, \mathrm{FBXO} 41^{+/-}$and FBXO41-/- $\mathrm{P} 16$ cortex and cerebellum, and did not detect any change (Fig. 3.20 A, B). In the next step, I cultured FBXO41 ${ }^{+/+}$, FBXO41 $1^{+/-}$and FBXO41-/- mouse CGNs and subjected them to 5 hours serum withdrawal (starvation) at DIV4 to examine if loss of FBXO41 rendered neurons more sensitive to stress-induced apoptosis. Subsequent immunoblotting of lysates revealed equal induction of CC3 expression (Fig. 3.20 C). Probing for the levels of phospho-ERK, totalERK, phospho-Akt and total-Akt in the FBXO41 ${ }^{+/+}, \mathrm{FBXO} 41^{+/-}$and $\mathrm{FBXO} 41^{-/-}$mice cortex and cerebellum led to similar negative results (Fig. 3.21). These data indicate that pathways regulating neuronal survival are not changed upon deletion of FBXO41.
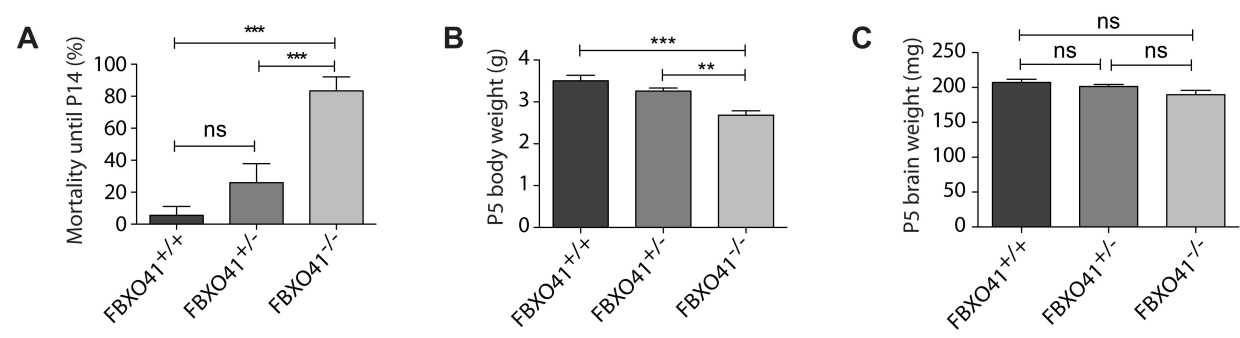

Figure 3.19.: FBXO41 ${ }^{-/-}$mice exhibit high mortality and reduced body and weight. (A) Mortality of the FBXO41 $1^{+/}, \mathrm{FBXO} 41^{+/-}$and $\mathrm{FBXO} 41^{-/-}$mice was analyzed in the first two postnatal weeks. (B, C) Body and brain weight of FBXO41 knockout mice was assessed at indicated ages. At least 10 animals of each genotype were analyzed. (ANOVA, ${ }^{* *} \mathrm{p}<0.01,{ }^{* * *} \mathrm{p}<0.001, \mathrm{~ns}=$ non-significant, mean + s.e.m.). 


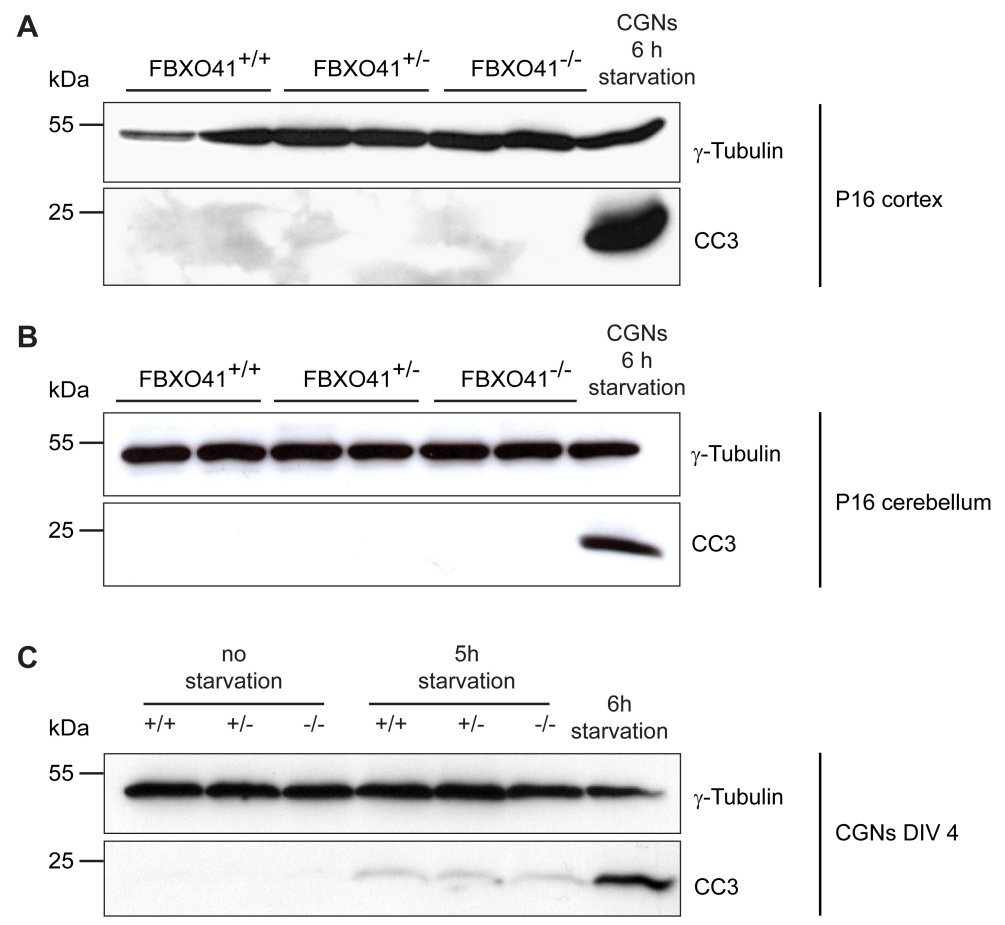

Figure 3.20.: FBXO41 knockout does not increase CC3 expression in mice. Lysates from the P16 FBXO41 ${ }^{+/+}, \mathrm{FBXO} 41^{+/-}$and FBXO41 ${ }^{-/-}$cortex and cerebellum (A, B) or from CGNs deprived of serum for 5 hours $(C)$ were immunoblotted with the CC3 antibody. Lysates from CGNs after 6 hours of serum deprivation served as a positive control for the CC3 expression. $\gamma$-Tubulin was used as a loading control. 
P16 cortex

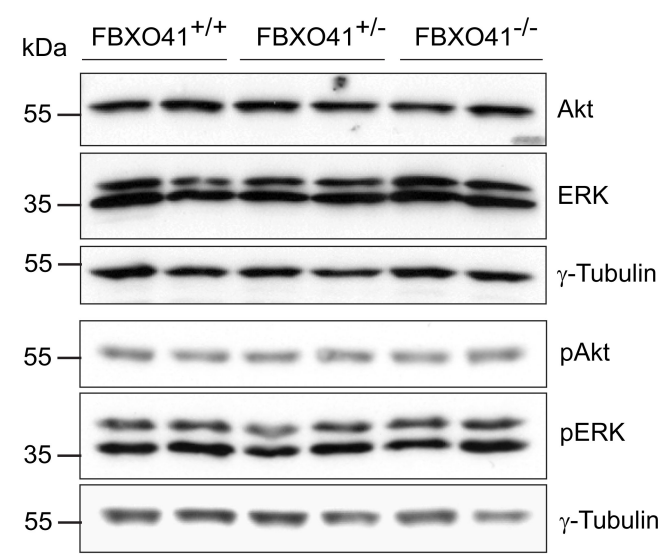

P16 cerebellum

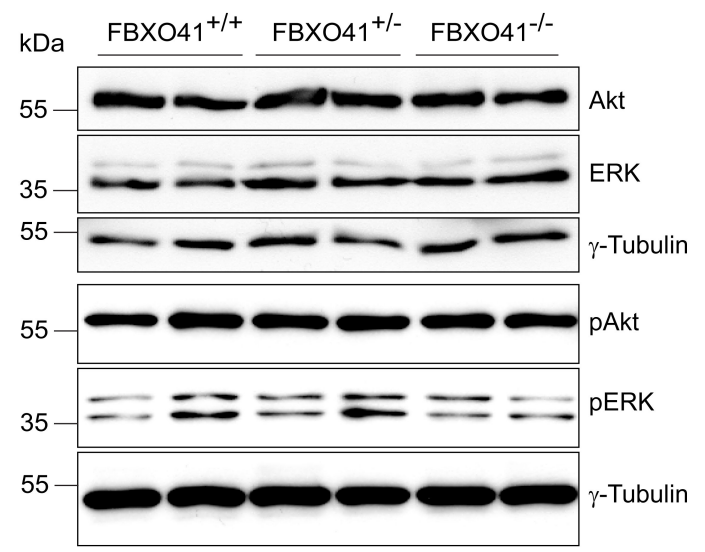

Figure 3.21.: ERK and Akt pathways are not affected by the loss of FBXO41 in mice. Levels of total Akt, total ERK, phospho-Akt and phospho-ERK were determined in the lysates from $\mathrm{P} 16 \mathrm{FBXO} 41^{+/+}, \mathrm{FBXO} 41^{+/-}$and $\mathrm{FBXO} 41^{-/-}$mice cortex and cerebellum by immunoblotting with the indicated antibodies. $\gamma$-Tubulin served as a loading control.

\subsubsection{Amino acids $179-354$ direct FBXO41 to the centrosome}

To investigate if the centrosomal localization of FBXO41 is essential for axon growth or migration control, I first identified the region that targets FBXO41 to the centrosome. I transfected both, HEK 293T cells and CGNs with a series of GFP-tagged FBXO41 deletion mutants (Fig. 3.22 A). Subsequently, I subjected the cells to immunostaining with the GFP antibody and observed that whenever the region between aa 179-354 is missing, FBXO41 did not localize to the centrosome (Fig. 3.22 B). The experiment thus identified this region of FBXO41 as the centrosome targeting region (CTR). 


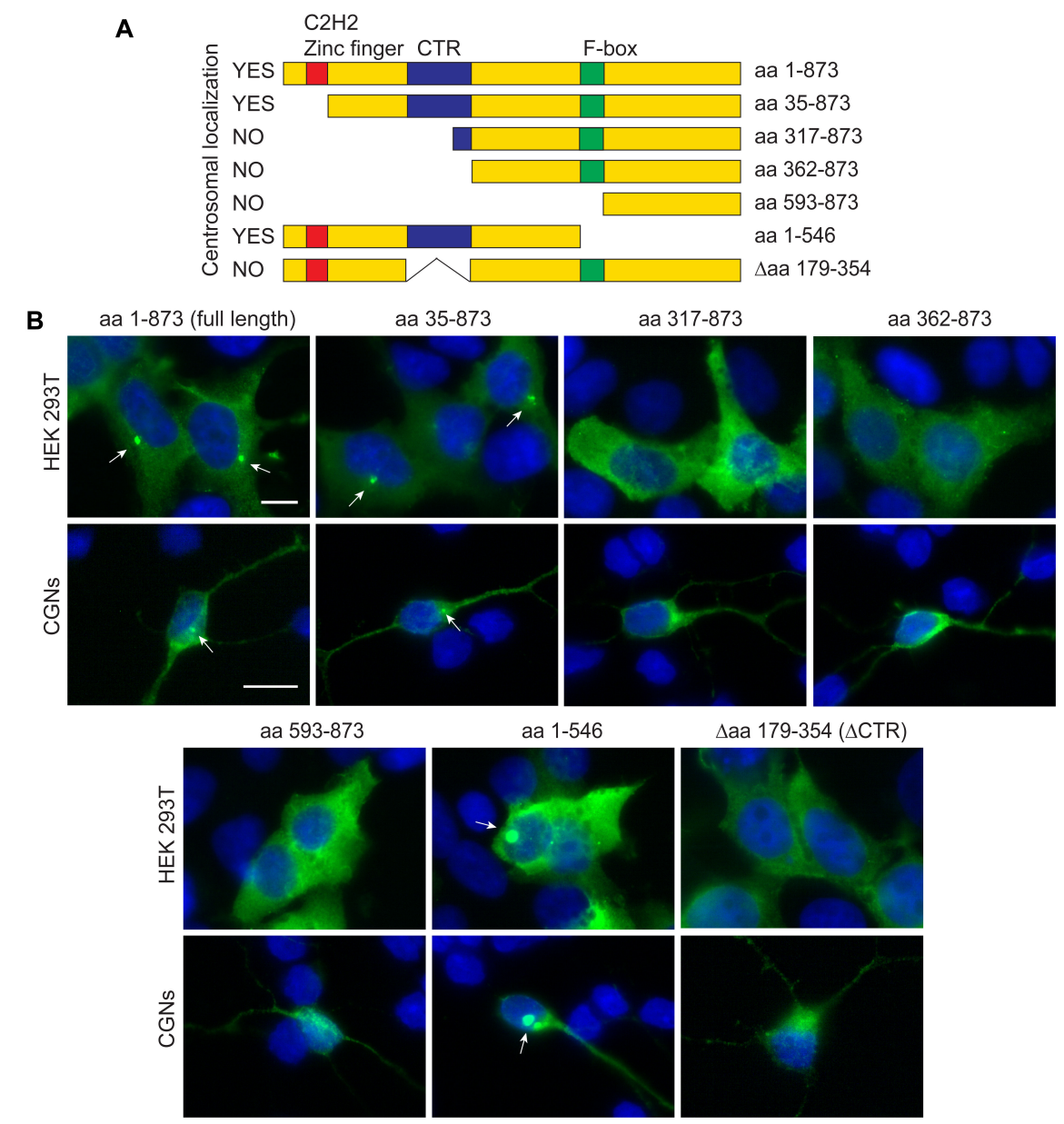

Figure 3.22.: Amino acids 179-354 target FBXO41 to the centrosome. (A) Schematic representation of various FBXO41 deletion mutants and their localization. The deletion mutants were cloned by N. Schwedhelm-Domeyer. (B) HEK 293T cells or CGNs were transfected with the GFP-tagged FBXO41 deletion mutants depicted in (A). After 2 days cells were subjected to immunostaining with the GFP antibody and the DNA dye bisbenzimide Hoechst 33258. Arrows indicate centrosomes. Scale bar: $10 \mu \mathrm{m}$. 


\subsubsection{FBXO41 regulates axon growth in a centrosome-independent manner}

To asses if centrosomal FBXO41 is responsible for promoting axon growth, I transfected CGNs with empty vector or plasmids encoding wild type FBXO41 or FBXO41 $\Delta \mathrm{CTR}$ and measured axon length after 3 days. Even though FBXO41 $\Delta$ CTR did not localize to the centrosome, it still promoted axon growth to the extend of the wild type FBXO41, suggesting that centrosomal localization is dispensable for the FBXO41mediated axon growth control (Fig. 3.23).

A

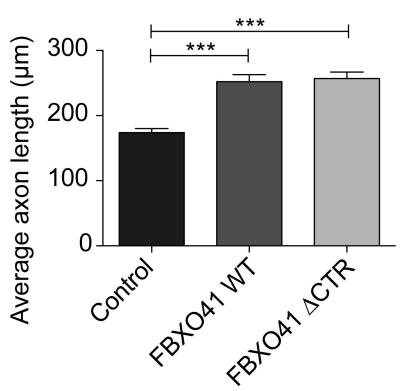

B

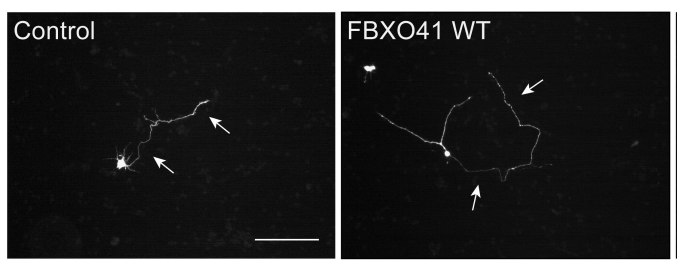

FBX041 $\triangle \mathrm{CTR}$

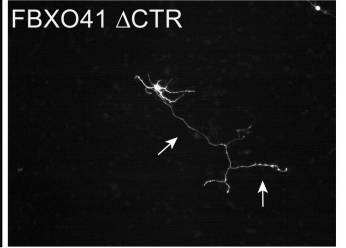

Figure 3.23.: Centrosomal localization is not required for the FBXO41-mediated axon growth regulation. (A) CGNs were transfected with the full length or FBXO41 $\triangle \mathrm{CTR}$ as well as with the empty control vector and morphometrically analyzed after 3 days. A total of 849 neurons was measured (ANOVA, ${ }^{* * *} \mathrm{p}<0.001$, mean + s.e.m.). (B) Representative images of (A). Arrows indicate axons. Scale bar: $100 \mu \mathrm{m}$.

In order to corroborate this result, I investigated if FBXO41 $\triangle \mathrm{CTR}$ was able to rescue the short axon phenotype caused by FBXO41 knockdown. For this purpose, I generated and validated an RNAi-resistant FBXO41, lacking the CTR (Fig 3.24) and carried out an axon growth assay, in which I expressed FBXO41-Res or FBXO41 $\Delta$ CTR-Res in the background of FBXO41 knockdown. Morphometrical analysis revealed that expression of both, wild type and $\triangle \mathrm{CTR}$ rescue plasmids restored the axon length of the FBXO41 knockdown neurons to the control level (Fig. 3.25). This experiment demonstrated that the acentrosomal FBXO41 promotes axon growth. 


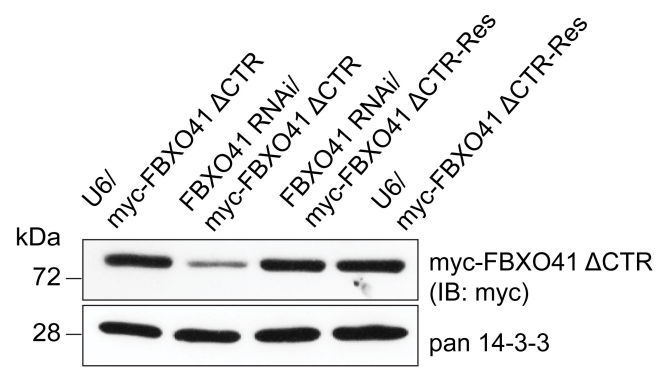

Figure 3.24.: FBXO41 $\triangle$ CTR-Res is resistant to the RNAi against FBXO41. HEK 293T cells were transfected with the indicated plasmids and lysed 4 days later. Lysates were subjected to immunoblotting using the myc antibody. Pan 14-3-3 served as a loading control.
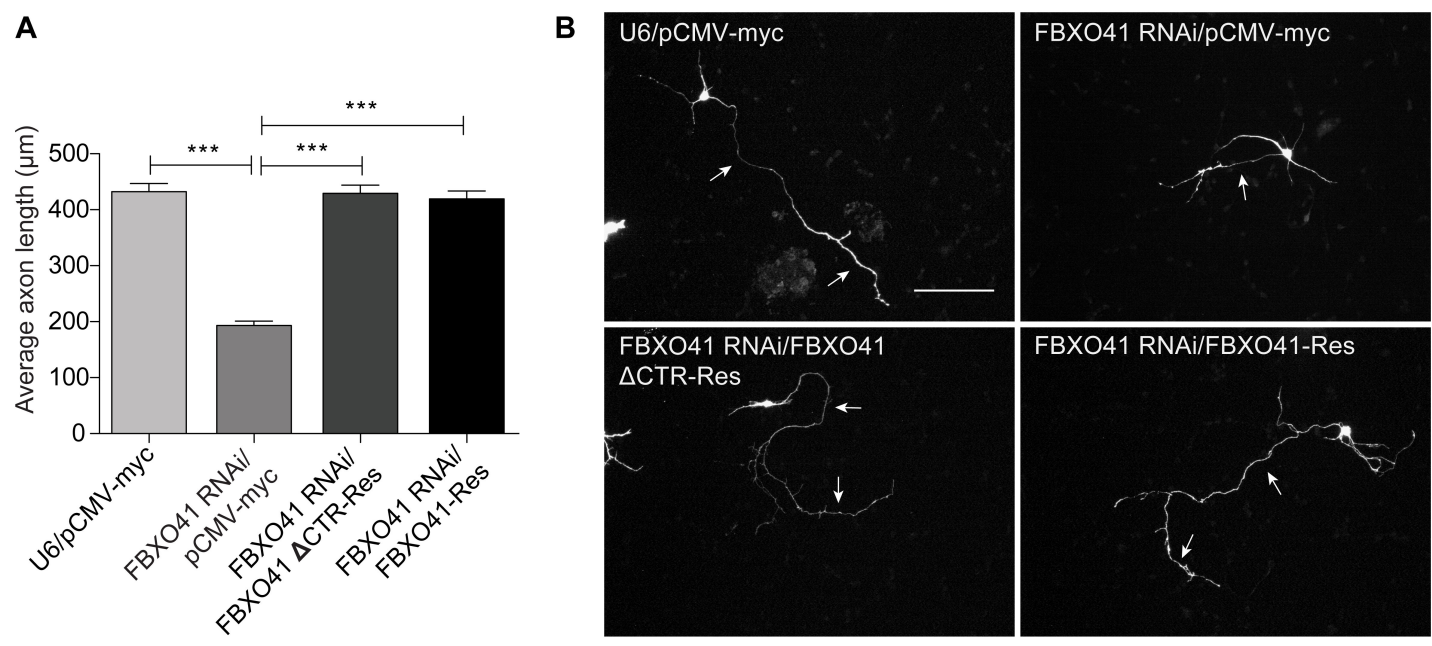

Figure 3.25.: FBXO41 $\triangle \mathrm{CTR}$ rescues the effect of FBXO41 knockdown on the axon growth. (A) CGNs were transfected with the indicated plasmids and axon length was measured 4 days later. 668 neurons were analyzed. (ANOVA, ${ }^{* * *} \mathrm{p}<0.001$, mean + s.e.m.). (B) Representative images of (A). Arrows indicate axons. Scale bar: $100 \mu \mathrm{m}$.

\subsubsection{Centrosomal FBXO41 promotes neuronal migration}

In further in vivo electroporation analyses, I investigated if FBXO41 regulates neuronal migration in a centrosome-dependent manner. Measurement of the migration distance of CGNs, electroporated with control vector or plasmids encoding FBXO41 WT 
or FBXO41 $\triangle \mathrm{CTR}$, showed that while the FBXO41 WT enhanced migration, FBXO41 $\triangle$ CTR CGNs migrated significantly shorter distances than FBXO41 WT neurons but significantly farther than control neurons (Fig. 3.26). This experiment suggests that the centrosomal localization of FBXO41 is required for proper CGN migration.

A

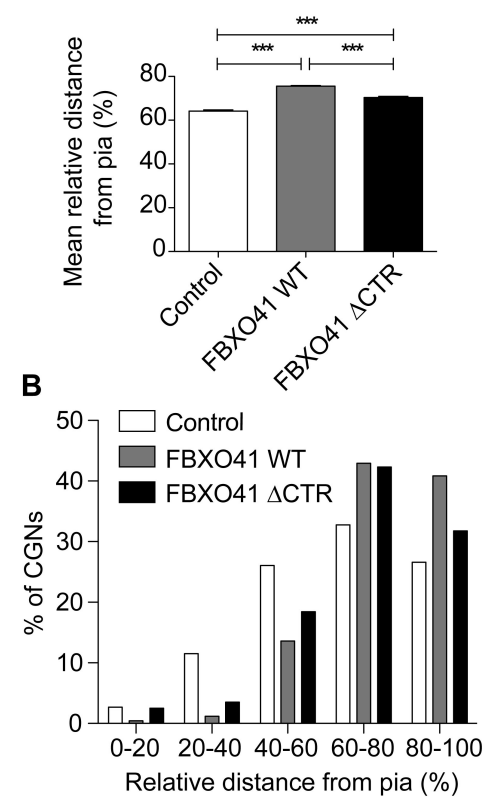

C

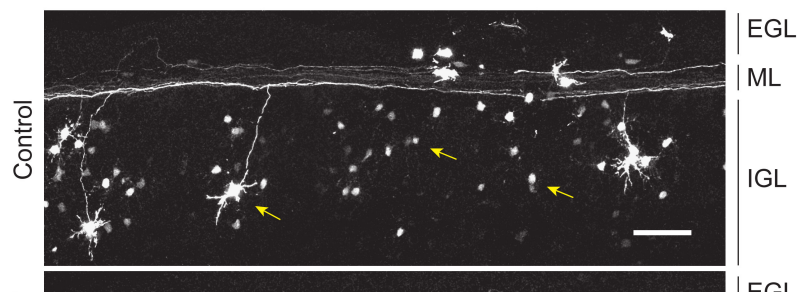

\begin{tabular}{l}
5 \\
\multirow{2}{5}{} \\
0 \\
0 \\
0 \\
0
\end{tabular}
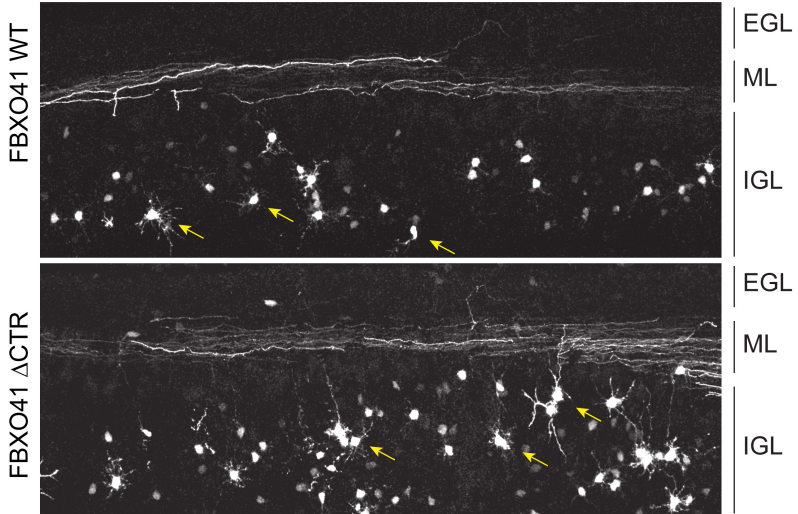

Figure 3.26.: FBXO41 promotes neuronal migration in a centrosome-dependent manner. (A, B) Cerebella of $\mathrm{P} 4$ rat pups were electroporated with control vector, FBXO41 or FBXO41 $\triangle$ CTR plasmid together with pSyn-GFP expression plasmid. 5 days later cerebella were isolated and coronal sections were immunostained with the GFP antibody. Mean migrated distance (A) and distribution (B) of GFP-positive CGNs were assessed. 4404 neurons were analyzed (Kruskal-Wallis, ${ }^{* * *} \mathrm{p}<0.001$, mean + s.e.m.) The statistical test was performed by Dr. S. Papiol. (C) Representative images of (A) and (B). Arrows indicate transfected CGNs. Scale bar: $50 \mu \mathrm{m}$. Dr. J. Stegmüller performed the in vivo electroporation and Dr. M. Vadhvani took the confocal images. 


\subsection{Molecular mechanism of FBXO41-mediated axon growth and neuronal migration}

Following the functional analyses, I proceeded with the dissection of the molecular mechanism, by which FBXO41 regulates neuronal migration and axon growth. Here I carried out a candidate approach to identify interactors of FBXO41 that cooperate in this process.

\subsubsection{FBXO41 does not form a canonical Cullin1-based SCF E3 ubiquitin ligase}

Most of F-box proteins serve as interchangeable substrate-recognition subunits of the Skp1-Cullin1-F-box (SCF) E3 ubiquitin ligases (Feldman et al., 1997, Skowyra et al., 1997). An interaction with both Skp1 and Cullin1 is required for F-box proteins to form a functional SCF complex (Zheng et al., 2002). To determine if FBXO41 forms a Cullin1-based SCF complex, we examined the interaction with Cullin1 and Skp1. Using heterologous cells, we found that FBXO41 associated with Skp1 and that this interaction depended on the F-box domain (Fig. 3.27 A).

Next, I investigated the FBXO41 and Cullin1 interaction. Again, using a heterologous cell system, I discovered that while the control F-box protein FBXO31 interacted with Cullin1, FBXO41 failed to associate with Cullin1 (Fig. 3.27 B). Thus, these experiments suggest that FBXO41 does not associate with Cullin1 to form a conventional SCF complex.

To date, among 8 known Cullins, only Cullin1 and Cullin7 (that binds to FBXW8) have been shown to form SCF complexes (Sarikas et al., 2008). Hence, we went on to investigate the interaction of FBXO41 and Cullin7 and demonstrated that both proteins interact in HEK 293T cells (C. Mukherjee, unpublished data). This indicates that FBXO41 is the second identified F-box protein forming a Cullin7-based E3 ubiquitin ligase. 

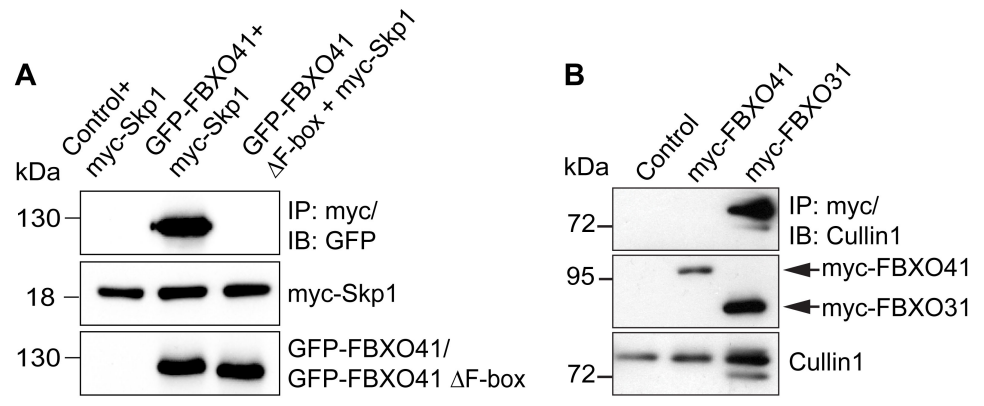

Figure 3.27.: FBXO41 interacts with Skp1 but not with Cullin1. (A) HEK 293T cells were transfected with the control plasmid, GFP-FBXO41 or GFP-FBXO41 $\Delta$ F-box together with myc-Skp1 and subjected to immunoprecipitation with the myc antibody. Lysates were subsequently probed with the GFP antibody. The experiment was performed by Dr. M. Kannan. (B) Lysates from HEK 293T cells transfected with mycFBXO41, myc-FBXO31 or empty control vector were immunoprecipitated with the myc antibody and immunoblotted with the Cullin1 antibody.

\subsubsection{FBXO41 is a novel interacting partner of DISC1}

To identify binding partners that act together with FBXO41 in the control axon growth and neuronal migration, we took a candidate approach and screened centrosomal proteins with a role in neural development. Among those, we found DISC1 to associate with FBXO41. Several genetic studies showed association of DISC1 with schizophrenia and other psychiatric disorders (Millar et al., 2000b, Ekelund et al., 2001, Hennah et al., 2003). In addition to that, DISC1 has been implicated in cell fate determination, regulation of neurite outgrowth and neuronal migration (Kamiya et al., 2006, 2005, Mao et al., 2009).

I established a robust interaction of FBXO41 and DISC1, using HEK 293T cells. Here, I transfected cells with Flag-DISC1 and GFP empty vector or GFP-FBXO41 and used the lysates to perform an immunoprecipitation with the GFP antibody, demonstrating that DISC1 indeed binds to FBXO41 (Fig. 3.28 A). Further, I confirmed this result by conducting a reciprocal co-immunoprecipitation, where I immunoprecipitated Flag-DISC1 and detected bound myc-FBXO41 (Fig. 3.28 B). To corroborate the finding that FBXO41 forms a complex with DISC1, I examined the localization of GFP-FBXO41 and Flag- 
DISC1 in HEK 293T and observed that both proteins colocalize at the centrosome and in the cytoplasm (Fig. $3.28 \mathrm{C}$ ). These results demonstrate that FBXO41 is an interactor of DISC1.

A

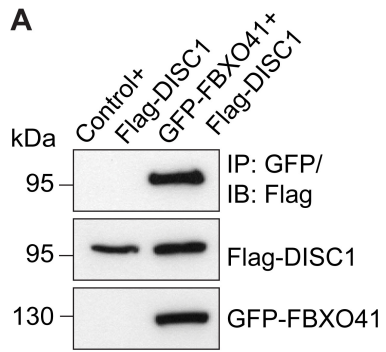

C

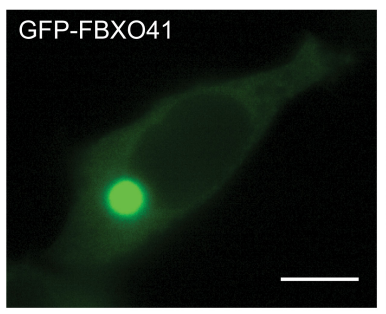

Flag-DISC1
B
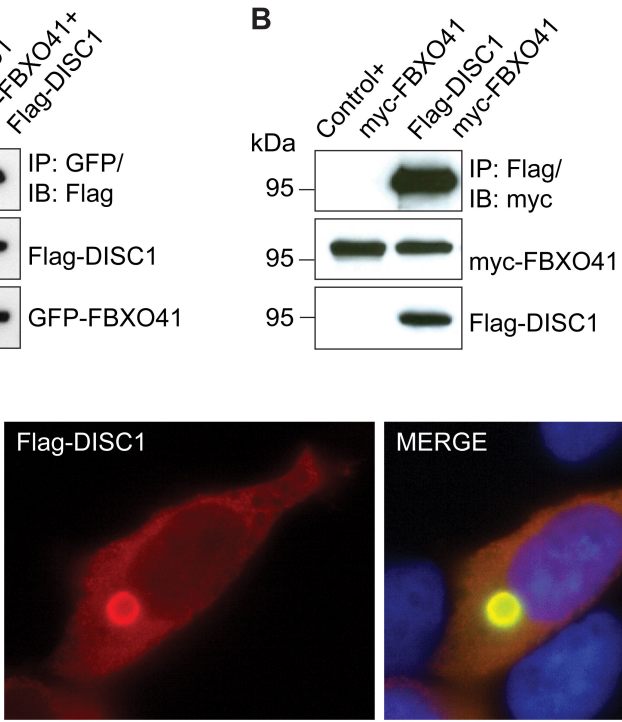

MERGE

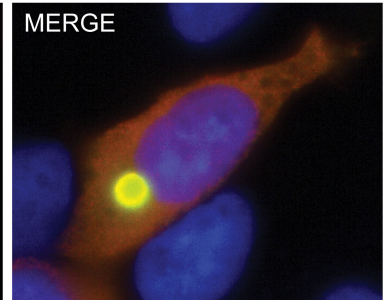

Figure 3.28.: FBXO41 interacts with DISC1. (A, B) HEK293T cells were transfected with the indicated plasmids and lysed 3 days later. $10 \%$ of the lysates were used as an input control. The remaining lysates were subjected to immunoprecipitation with the GFP (A) or Flag (B) antibodies and subsequently immunoblotted with the indicated antibodies. (C) HEK 293T cells were transfected with the GFP-FBXO41 plasmid together with the Flag-DISC1. Cells were subjected to immunocytochemistry using the Flag and GFP antibodies and the DNA dye bisbenzimide Hoechst 33258. Scale bar: $10 \mu \mathrm{m}$.

\subsubsection{DISC1 mediates the interaction between FBXO41 and NDEL1}

Strikingly, I also found that another centrosomal protein, implicated in migration, associates with FBXO41 via DISC1. I examined if FBXO41 forms a complex with DISC1 and NDEL1 in HEK 293T cells and observed that FBXO41 can be immunoprecipitated with NDEL1 in the presence of DISC1. (Fig. 3.29). This finding establishes DISC1 as a 
link between FBXO41 and NDEL1.

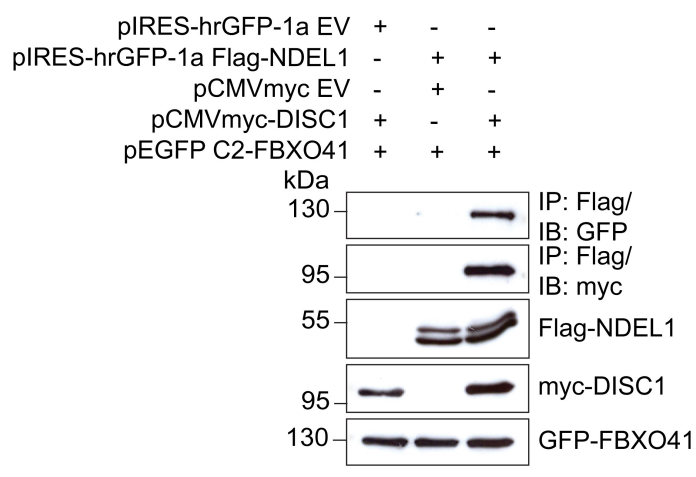

Figure 3.29.: FBXO41 interacts with NDEL1 in the presence of DISC1. HEK 293T cells were transfected with indicated plasmids and lysed 2 days later. The lysates were subsequently immunoprecipitated with the Flag antibody and immunoblotted with the myc and GFP antibodies.

\subsubsection{DISC1 is not a proteolytic target of FBXO41-Cullin7 SCF complex}

Since FBXO41 forms a Cullin7-based SCF complex, the question arose if FBXO41 ubiquitinates DISC1, and possibly targets it for proteasomal degradation. I first tested if DISC1 accumulates in the FBXO41-/- brain and I subjected lysates from P16 cerebella and cortices to immunoblotting with the DISC1 antibody but did not observe a difference in the protein expression level of wild type as compared to FBXO41 knockout (Fig. 3.30 A, C). Immunoblotting with the antibody that was preincubated with the blocking peptide, verified the specific detection of DISC1 (Fig. 3.30 B). This experiment suggests that DISC1 is not targeted for proteasomal degradation by FBXO41.

Apart from proteasomal degradation, ubiquitination can lead to functional modifications (Chau et al., 1989, Galan and Haguenauer-Tsapis, 1997, Chen et al., 1996). In that case, levels of the targeted protein may not change. For this reason, I investigated if DISC1 can be a target of the FBXO41-SCF complex, by performing a CoIP of Cullin7 and DISC 1 in the presence or absence of FBXO41. The experiment demonstrated lack 
A
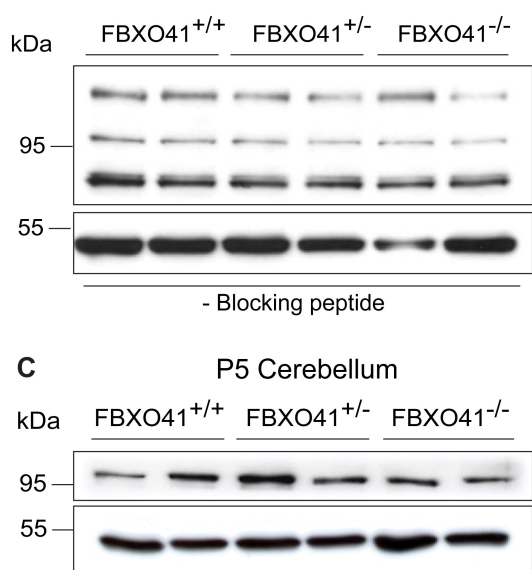

B

$\underline{\mathrm{FBXO}_{4} 1^{+/+}} \mathrm{FBXO41}^{+/-} \mathrm{FBXO41^{-/- }}$ P16 Cortex

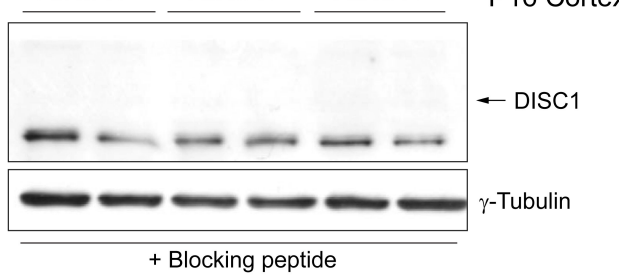

D P16 Cerebellum

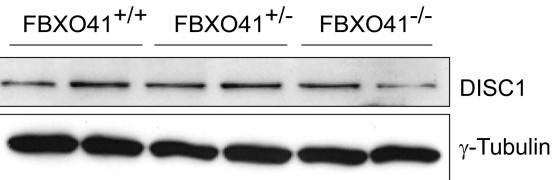

Figure 3.30.: DISC1 does not accumulate in the FBXO41- ${ }^{-/}$mice. (A, B, C, D) Lysates from cortex and cerebellum of indicated ages were immunoblotted with the DISC1 antibody (A, C, D) or with the DISC1 antibody that was preincubated with a blocking peptide (B). $\gamma$-Tubulin antibody was used as a loading control.

of physical association of DISC1 and Cullin7-FBXO41 complex, suggesting that DISC1 is not a target of the E3 ligase (Fig 3.31).

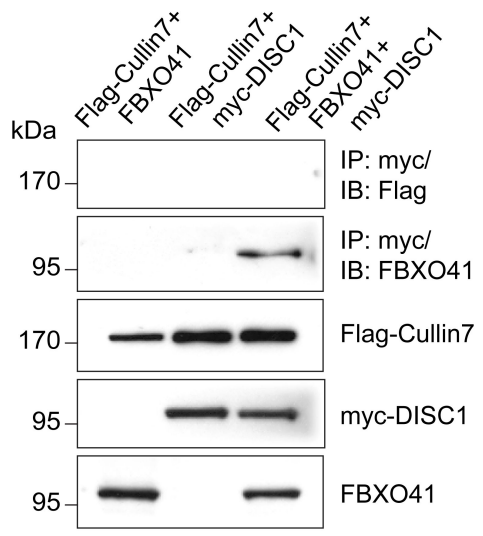

Figure 3.31.: Cullin7 does not associate with DISC1. Lysates from HEK 293T cells transfected with indicated plasmids were immunoprecipitated with the myc antibody and probed with the Flag and FBXO41 antibodies. 


\subsubsection{NDEL1 accumulates upon FBXO41 knockout}

To investigate if FBXO41 mediates ubiquitination and degradation of NDEL1, I analyzed NDEL1 expression in the FBXO41 $1^{+/}, \mathrm{FBXO} 41^{+/-}$and $\mathrm{FBXO} 41^{-/-}$mice. Immunoblotting with the NDEL1 antibody did not show any change across genotypes at P16 but an accumulation of NDEL1 was observed in at P5 FBXO41 $1^{+/-}$and FBXO41 ${ }^{-/-}$ cerebella (Fig. 3.32). This result suggests that the FBXO41 affects the levels of NDEL1 in mouse cerebellum at an age, which is crucial for neuronal migration.

P5 Cerebellum

P16 Cerebellum
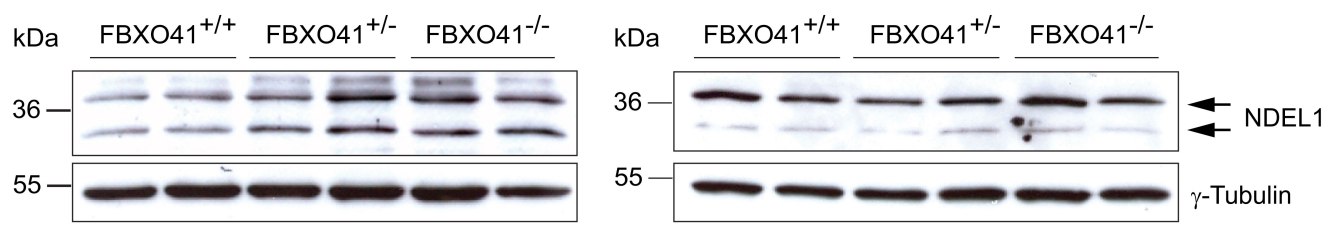

Figure 3.32.: FBXO41 affects expression levels of NDEL1 in P5 cerebellum. Lysates

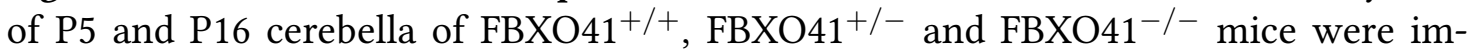
munoblotted with the NDEL1 and the $\gamma$-Tubulin antibodies. The latter served as a loading control.

\subsubsection{The CTR domain of FBXO41 mediates the interaction with DISC1}

To determine if FBXO41 controls axon growth and neuronal migration in a DISC1-dependent manner, we used GFP-tagged FBXO41 truncation constructs and myc-DISC1 to transfect HEK293T prior to immunoprecipitation with the GFP antibody. Subsequent immunoblotting with the myc antibody showed that only the deletion mutants of FBXO41 that lack the C-terminal fragment of the CTR did not interact with DISC1, identifying this stretch as DISC1-binding region (Fig. 3.33). 
A

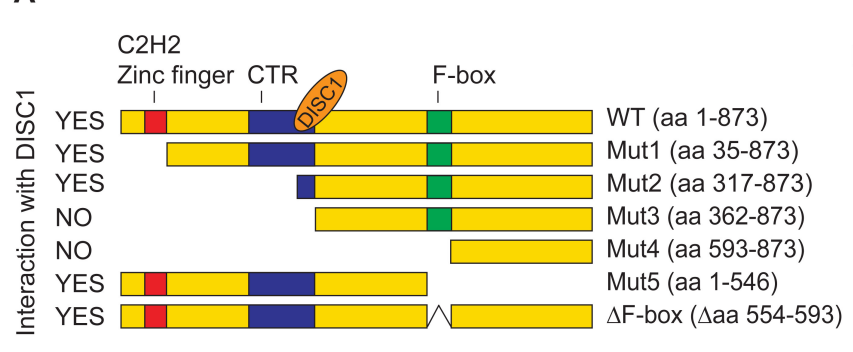

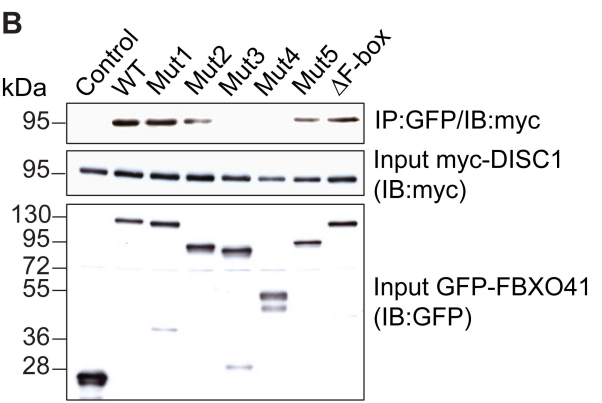

Figure 3.33.: DISC1 binds to the CTR of FBXO41. (A) Schematic of various deletion mutants of FBXO41 and their interaction with DISC1. (B) HEK293T cells transfected with the GFP-tagged deletion mutants of FBXO41 together with the myc-DISC1 were lysed 3 days later and subjected to immunoprecipitation with the GFP antibody and detection with the myc antibody. This experiment was performed by Dr. J. Stegmüller and Dr. M. Kannan.

\subsubsection{FBXO41 and DISC1 act in parallel pathways to control axon growth}

Having established the CTR as binding region of DISC1, this also led to the conclusion that FBXO41 $\triangle$ CTR-stimulated axon growth is DISC1-independent (see sections 3.2.4 and 3.2 .5 for the details).

To gain further insight into the FBXO41-DISC1 interaction in axon growth, I performed epistasis analysis by overexpressing FBXO41 and downregulating DISC1 in CGNs. For this purpose D. Brockelt generated a DISC1 RNAi and I verified the efficiency of the knockdown by transfecting HEK 293T cells with myc-DISC1 construct together with either the U6 empty vector or the RNAi vector. Subsequently, I immunoblotted the lysates with the myc antibody and observed a reduction in DISC1 level upon overexpressing the RNAi (Fig. 3.34 A). Analysis of CGNs, transfected with the control plasmids, the FBXO41 plasmid, the DISC1 RNAi plasmid or with the FBXO41 together with the DISC1 RNAi plasmids, revealed that while FBXO41 overexpression increased axon length, DISC1 knockdown resulted in a decrease. The axon length of neurons expressing both plasmids was similar to control neurons. These findings suggest that 
FBXO41 and DISC1 regulate axon growth independent from each other (Fig. 3.34 B, C).
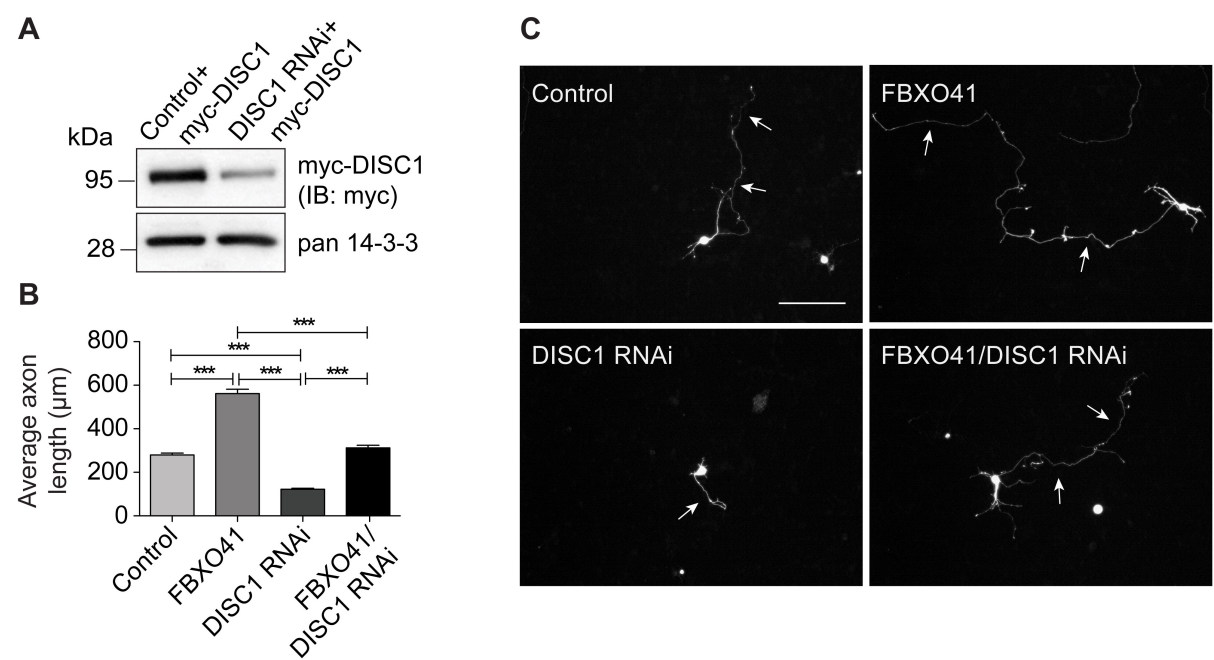

Figure 3.34.: DISC1 and FBXO41 act in separate pathways to control axon growth. (A) HEK 293T cells were transfected with the control vector and the DISC1 RNAi together with myc-DISC1. Lysates obtained after 4 days were probed with the myc antibody. Pan 14-3-3 served as a loading control. (B) CGNs were transfected with empty control vectors, DISC1 RNAi, myc-FBXO41, or both plasmids together and axon length was measured after 4 days. A total of 763 neurons was analyzed. (ANOVA, ${ }^{* *} \mathrm{p}<0.001$, mean + s.e.m.). (C) Representative images of (B). Arrows indicate axons. Scale bar: 100 $\mu \mathrm{m}$.

\subsubsection{DISC1-binding is essential for FBXO41-mediated control of neuronal migration}

As I demonstrated before (see section 3.2.6), the migration-promoting effect of FBXO41 requires the presence of the CTR, indicating that the interaction with DISC1 is also important for this process. In order to corroborate this finding, I generated and validated a bicistronic DISC1 RNAi plasmid that co-expressed EGFP to visualize transfected neurons (Fig. 3.35).

Next, we electroporated P4 rat pups with the control plasmid, FBXO41 RNAi, DISC1 


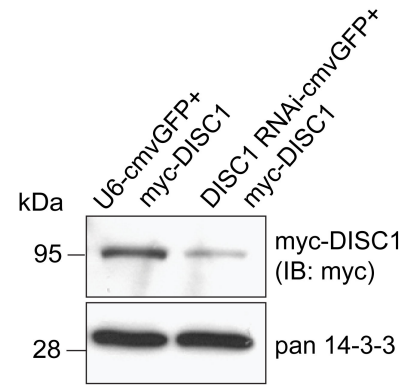

Figure 3.35.: Bicistronic RNAi downregulates DISC1 protein level. (A) HEK 293T cells transfected with the indicated plasmids were lysed 4 days later and subjected to immunoblotting with the myc and pan 14-3-3 antibodies. The latter served as a loading control.

RNAi or FBXO41 RNAi and DISC1 RNAi together. After 5 days, the measurement of distance the CGNs migrated showed that while, FBXO41 RNAi and DISC1 RNAi significantly inhibited migration, simultaneous knockdown of FBXO41 and DISC1 decreased migration even further (Fig. 3.36). This result suggests that FBXO41 and DISC1 functionally interact to regulate neuronal migration. 
A

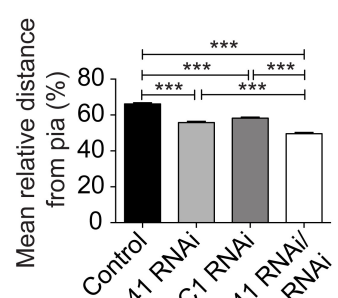

B

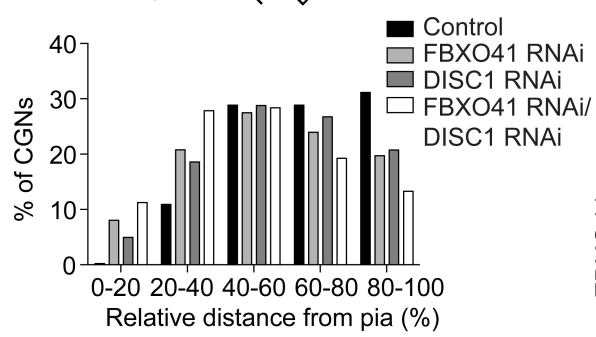

C
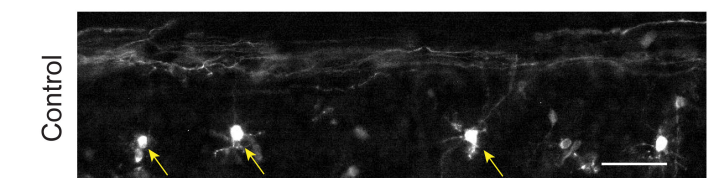

EGL

$\mathrm{ML}$

IGL

EGL

$M L$

IGL

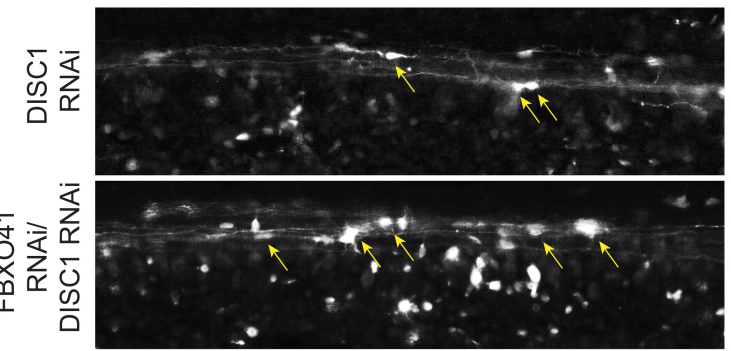

EGL $\mathrm{ML}$

IGL

EGL $\mathrm{ML}$ IGL

Figure 3.36.: FBXO41 and DISC1 cooperate to regulate neuronal migration. (A, B) Rat pups were electroporated at postnatal day 4 with U6-CMV-EGFP (Control), U6-FBXO41RNAi-CMV-EGFP (FBXO41 RNAi) or U6-DISC1RNAi-CMV-EGFP (DISC1 RNAi) plasmids. 5 days following the electroporation, cerebella were isolated, cut coronally and stained with the GFP antibody to visualize transfected neurons. Mean migrated distance (A) or distribution within the cerebellar cortex (B) were determined for a total of 4282 neurons (ANOVA, ${ }^{* * *} \mathrm{p}<0.001$, mean + s.e.m). (C) Representative images of (A) and (B). Arrows indicate transfected neurons. Scale bar: $50 \mu \mathrm{m}$. The in vivo electroporation was performed by Dr. J. Stegmüller. 



\section{Discussion}

During brain development, newborn neurons and neuronal precursors migrate considerable distances and undergo complex morphological changes to generate functional neuronal circuits. The importance of these processes is substantiated by the fact that defects in neuronal migration or axon growth result in serious neurodevelopmental disorders (Gleeson, 2001, Kato and Dobyns, 2003, Lewis and Levitt, 2002, Oprea et al., 2008). Thus, it is not surprising that the regulation of brain development is governed by external cues and cell autonomous pathways. While extrinsic mechanisms in the control of neurodevelopment have been widely described, intrinsic programs, including the ubiquitin-proteasome system, are beginning to shed further light onto regulation of developmental events in neurons.

Although the UPS represents a universal cellular machinery, more than 600 different E3 ubiquitin ligases encoded by the human genome allow for a temporally and spatially controlled regulation of specific events in the brain (Scheffner et al., 1995). Owing to a large number of F-box proteins, SCF complex ligases are extremely versatile (Deshaies, 1999) and have attracted a lot of attention in the cell cycle and cancer field (Skaar et al., 2009, Frescas and Pagano, 2008, Welcker and Clurman, 2008). Only recently, studies have examined various F-box proteins in the nervous system and revealed significant roles in brain development and in disease (Westbrook et al., 2008, Wu et al., 2013, Jandke et al., 2011, Fonzo et al., 2009, Watanabe et al., 2013, Litterman et al., 2011). Hence, F-box proteins could represent therapeutic targets for disease treatment, as different approaches have been developed in the past few years (Wu et al., 2012b, Orlicky et al., 2010, Aghajan et al., 2010, Sakamoto et al., 2001).

FBXO41 is a previously uncharacterized F-box protein, whose mRNA is highly ex- 
pressed in several regions of the brain. This study revealed the neuron-specific expression of FBXO41 in the CNS and its localization at the centrosome and in the cytoplasm. Moreover, FBXO41 is crucial to cerebellar development as it regulates neuronal migration and axon growth. Further analysis established the FBXO41/DISC1/NDEL1 interaction, which is important for FBXO41-mediated neuronal migration.

\subsection{FBXO41 is localized to the centrosome and the cytoplasm of CNS neurons}

FBXO41 has been identified as a brain specific F-box protein by the Cerebellar Development Transcriptome Database (CDT-DB). Reverse trancriptase PCR (RT-PCR) carried out in the lab, confirmed the presence of FBXO41 in CNS regions and its absence from non-neural tissue.

Since the available data on FBXO41 was restricted to its mRNA levels, we generated a polyclonal antibody to study the protein expression. The expression profile, which included neural and non-neural tissues, was consistent with the transcript data. The temporal expression pattern in the mouse cerebellum, obtained from the CDT-DB, indicated a peak in the FBXO41 transcript level at postnatal day 14. As I observed a similar increase of the protein level in the cerebellum, one can assume that FBXO41 expression is mainly transcriptionally regulated. Moreover, the upregulation of FBXO41 expression in the mouse embryo suggested a role in neural development. Additionally its continuous expression until the adulthood suggests, that FBXO41 may have further functions in synapse formation or maintenance of neuronal networks.

Among different cell types populating the brain, FBXO41 expression is only detectable in neurons, but not in oligodendrocytes or in astrocytes. Consistent with this finding, during cerebellar development, the FBXO41 transcript is found in the external granule layer at P7 and in the internal granule layer at P21. This expression pattern corresponds to the position of cerebellar granule neurons at the above mentioned developmental stages, underscoring the neuron-specific FBXO41 expression.

To characterize the expression of FBXO41 in more detail, we analyzed its subcellu- 
lar localization in neurons. Therefore, we used immunostaining of CGNs expressing a fluorescently-tagged FBXO41 and observed that it was localized in the axonal and dendritic compartment, and at the centrosome. We confirmed this result by centrosomal purification, which demonstrated that FBXO41 was present both in the cytoplasmic and in the centrosomal fraction. Since many centrosomal proteins, such as DISC1, NDEL1, LIS1, DCX have been implicated in neuronal migration (Morris et al., 2003, Kamiya et al., 2005, Sasaki et al., 2000, Feng et al., 2000, Tanaka et al., 2004), the centrosomal localization of FBXO41 indicated its possible role in this process.

\subsection{FBXO41 promotes neuronal migration in the cerebellar cortex}

Neuronal migration is essential for the establishment of proper wiring and connectivity of the brain and needs to be tightly controlled. Although several proteins involved in governing this process have been described, the exact molecular pathways remain elusive. Identification of new regulatory proteins could help decipher mechanisms underlying neuronal migration. In recent years several F-box proteins, such as FBXO31, FBXO45 and FBXW7 have been shown to be implicated in neuronal migration (Vadhvani et al., 2013, Saiga et al., 2009, Jandke et al., 2011). Due to the centrosomal localization of FBXO41, we decided to investigate its role in neuronal migration. Using in vivo electroporation of rat cerebella, we demonstrated, that knockdown of FBXO41 dramatically stalled migrating CGNs. Conversely, migration of CGNs was enhanced by overexpression of FBXO41.

Given the importance of the centrosome for neuronal migration, we examined if the centrosomal localization of FBXO41 is essential to regulate migration. We utilized the the in vivo electroporation system to overexpress FBXO41 $\triangle \mathrm{CTR}$ in CGN progenitors and monitor their migration. The non-centrosomal FBXO41 was not able to promote neuronal migration to the extend of the wild type protein. This finding places FBXO41 among the regulators of neuronal migration, whose function is centrosome-dependent. For instance, disruption of the centrosomal localization of katanin p60 and its aberrant 
nuclear distribution coincides with defects in migration (Toyo-Oka et al., 2005). Furthermore, overexpression of the polarity protein PAR6 $\alpha$, causes redistribution of centrosomal components, like $\gamma$-Tubulin, pericentrin, centrin2, PKC $\zeta$ and PAR6 $\alpha$, as well as disrupted neuronal migration (Solecki et al., 2004). Similarly, the loss of centrosomal localization of FBXO41 interferes with its ability to promote neuronal migration.

However, it is noteworthy that, even though not localized at the centrosome, the FBXO41 $\triangle$ CTR was still able to enhance neuronal migration to a limited extend. It suggest additional, acentrosomal mechanisms of FBXO41-mediated migration. This is consistent with the observation of Umeshima and colleagues that during radial migration of CGNs in organotypic cerebellar slices, the centrosome does not always precede the nucleus and that the nuclear forward movement can occur without the centrosome entering the leading process first (Umeshima et al., 2007). The suggested mechanism of the nuclear movement along the microtubules, into the leading process, involves the molecular motor activity of the LIS1/dynein complex, localized at the nuclear envelope (Tanaka et al., 2004, Tsai and Gleeson, 2005). Non-muscle myosin II acts behind the nucleus, to push it forward (Bellion et al., 2005, Zhu et al., 2010). Moreover, non-muscle myosin II has also been reported to localize in front of the nucleus, presumably pulling it towards the migratory direction (Solecki et al., 2009). It remains to be determined, if the acentrosomal FBXO41 regulate neuronal migration by any of this mechanisms.

During this study, we performed a candidate approach to identify interactors of FBXO41 and its potential ubiquitination targets, that would cooperate with FBXO41 in regulation of neuronal migration. We screened for proteins that exhibit centrosomal and cytoplasmic localization and are expressed from the embryonic development to adulthood. Furthermore, the candidates were required to have a function in neuronal migration and morphogenesis. As a consequence, we identified DISC1 as a binding partner of FBXO41. We determined the interaction of FBXO41 and DISC1 by the co-immunoprecipitation method, and we observed the colocalization of FBXO41 and DISC1 in HEK 293T cells. This finding is consistent with the results of a yest two-hybrid screen, that found FBXO41 as one of the candidate interactors of DISC1. However, that study did not provide any independent evidence for this association (Camargo et al., 
2007).

The fact that the DISC1 locus has been linked to schizophrenia and related mental disorders (Ekelund et al., 2001, 2004, Hamshere et al., 2005, Hwu et al., 2003, Macgregor et al., 2004) prompted many groups to generate DISC1 mouse models. It is however impossible to study the full spectrum of schizophrenia symptoms in mice. For example, some positive symptoms, like delusions and hallucinations cannot be modeled in animals. Nevertheless, it is still possible to analyze several negative and cognitive hallmarks in mouse models (Arguello and Gogos, 2006). So far it is not clear what is the consequence of the balanced translocation in the Scottish family for the expression of DISC1: it may result in haploinsufficiency or the C-terminally truncated protein could be expressed and constitute a dominant negative variant of DISC1. Several mouse models expressing truncated versions of DISC1 have been generated until now. Behavioral deficits, corresponding to those observed in schizophrenia patients, have been reported in these mice. Some of them include prolonged immobility in the forced swim test, hyperactivity, decreased prepulse inhibition. Additionally, males exhibited decreased social interactions and females showed deficits in working memory (Hikida et al., 2007, Pletnikov et al., 2008). Some of the animal models display missense mutations within DISC1 sequence, such as L100P and Q31L substitutions. Mice with the L100P mutation exhibit more schizophrenia-like phenotype, including deficiency in prepulse inhibition and latent inhibition, which were reversible upon treatment with antipsychotics. Mice with the Q31L mutation in turn, exhibit more depression-like behavior, with not so pronounced deficit in prepulse inhibition, but with shortcoming in latent inhibition and the forced swim test(Clapcote et al., 2007). Interestingly, FBXO41 locus has been found within one of the genomic regions, that have been linked to schizophrenia (Nato et al. 2006). Moreover, a functional gene group analysis demonstrated that a group of synaptic genes containing FBXO41 was associated with the risk of schizophrenia (Lips et al., 2012). In this context, it would be interesting to perform the schizophrenia-relevant behavioral tests such as prepulse inhibition to analyze the sensory gating, 5-choice serial reaction time task and latent inhibition to test attentional deficits, delayed nonmatch to position or T maze test to determine the working memory deficits, forced swim test to 
examine depression as well as novel object recognition task, which can be used to determine functions related to hippocampus and cortex, especially visual working memory (Arguello and Gogos, 2006, Niwa et al., 2010) on the FBXO41-deficient mice. Since the FBXO41 knockout mice exhibit a severe motor phenotype, using the heterozygous animals would probably ensure more reliable results.

DISC1 has been shown to promote migration of neurons in the developing brain, but appears to restrict the speed of neuronal integration in the adult hippocampus (Duan et al., 2007). Aside from neuronal migration in the cortex, DISC1 controls migration of granule and pyramidal neurons in the hippocampus (Kamiya et al., 2005, Meyer and Morris, 2009, Tomita et al., 2011). Consistently with these data, I found that knockdown of DISC1 inhibits migration of cerebellar granule neurons, which is similar to the effect of FBXO41 knockdown. I also discovered that the double knockdown of FBXO41 and DISC1 led to a more pronounced phenotype. A study from Duan and colleagues demonstrated that another binding partner of DISC1 - NDEL1, also showed an additive effect of migration inhibition, when downregulated together with DISC1. This finding implied that NDEL1 and DISC1 functionally interact to regulate migration (Duan et al., 2007). Along that line my data suggests that FBXO41 may control neuronal migration together with DISC1. Further in vivo electroporation experiment overexpressing FBXO41 WT or the $\triangle \mathrm{CTR}$ mutant, that does not bind to DISC1 supported this conclusion. This result demonstrated that the interaction with DISC1 is crucial for FBXO41 to properly support neuronal migration. The finding that FBXO41 has residual activity to promote neuronal migration without interacting with DISC1 could imply that FBXO41 controls neuronal positioning by an additional parallel pathway that may involve microtubule binding proteins (Bielas et al., 2004).

DISC1 regulates neuronal migration by interacting with a variety of proteins, including BBS4 (Kamiya et al., 2005), CAMD1 (Fukuda et al., 2010), kinesin-1 (Kamiya et al., 2005) and probably the best characterized NDEL1-LIS1-dynein complex (Duan et al., 2007, Torisawa et al., 2011). In the present study, I demonstrated an interaction of FBXO41 with NDEL1, that occurs in the presence of DISC1. Phosphorylation of both, DISC1 and NDEL1 is necessary for their function in neuronal migration. DISC1 be- 
comes phosphorylated at serine 710 to switch from a regulator of proliferation to migration (Ishizuka et al., 2011b). The phosphorylation of NDEL1 by Cdk5 (cyclin-dependent kinase 5) promotes NDEL1's interaction with katanin p60. This ensures the proper localization of NDEL1, which is necessary for neuronal migration (Toyo-Oka et al., 2005). By associating with DISC1 and NDEL1, FBXO41 might affect their phosphorylation state in the control of neuronal migration. Examining the levels of phosphorylated DISC1 or NDEL1 in the FBXO41 knockout mice, using the phospho-specific antibodies would shed more light on that subject.

NDEL1 interacts with LIS1 to regulate cytoplasmic dynein during migration (Torisawa et al., 2011). It would therefore be of great relevance to examine if FBXO41 acts on dynein activity. An established method to analyze dynein function is to monitor the transport of its cargo in living cells. This approach would also be useful to assess dynein activity in the neurons devoid of FBXO41.

Apart from regulating the transport of cellular cargo during migration, the NDEL1LIS1-dynein complex has been also implicated in nuclear translocation and centrosomenucleus coupling (Shu et al., 2004). Hence, analysis of the nuclear movement and the distance between nucleus and the centrosome in FBXO41-depleted neurons would provide a deeper insight into the mechanisms underlying FBXO41 function in neuronal migration.

FBXO41 may also regulate neuronal migration by modulating the actin cytoskeleton. It has been reported that NDEL1 is necessary for activating the Rho GTPase Cdc42 at the leading edge of migrating neurons (Shen et al., 2008). Activity levels of Cdc42 FBXO41 knockout neurons might thus provide an indication whether FBXO41 is involved in NDEL1-mediated regulation of Cdc42.

NDEL1 and its interacting partners have been implicated in several other molecular pathways that control neuronal migration. For instance, migration defects and increased distance between the centrosome and the nucleus observed in the LIS1 $1^{+/-}$neurons can be rescued by overexpression of DCX (Doublecortin). This suggests a functional cooperation of both proteins (Tanaka et al., 2004). In migrating neurons, the $\mathrm{PAR} 6 \alpha / \mathrm{PKC} \zeta$ activity is localized at the centrosome and controls its movement in the 
direction of migration. Overexpression of PAR6 $\alpha$ disrupts the centrosomal localization of proteins like $\gamma$-Tubulin, pericentrin, centrin2, $\operatorname{PKC} \zeta$ as well as PAR6 $\alpha$ itself and inhibits neuronal migration (Solecki et al., 2004). Additional investigation would be necessary to determine if some of the aforementioned regulators cooperate with FBXO41 to form a regulatory pathways governing neuronal migration.

\subsection{FBXO41 forms a Cullin7-based E3 ubiquitin ligase}

In order to identify mechanisms by which FBXO41 regulates neuronal migration, I addressed if FBXO41, similar to other F-box proteins, is part of an SCF E3 ligase and ubiquitinates proteins involved in the regulation of neuronal motility. Co-immunoprecipitation experiments performed in this study demonstrated, that FBXO41 interacts with Skp1 and this interaction is F-box domain-dependent. However, unlike most F-box proteins, FBXO41 does not associate with Cullin1 to form an SCF complex. Analysis of the crystal structure of SCF ${ }^{\text {Skp2 }}$ complex revealed that Skp2 interacts with Cullin1 not only via Skp1, but also directly through two amino acids within the F-box domain (P113 and E115 in case of Skp2) (Zheng et al., 2002). As it has been demonstrated for the F-box protein FBXO45, reconstitution of only one of these crucial amino acids can restore the interaction with Cullin1 (Saiga et al., 2009). Alignment of several Cullin1-binding F-box proteins and FBXO41 revealed that the crucial proline and glutamic acid are replaced by arginine and alanine in FBXO41. This results in a distinct interface for Cullin1 binding to FBXO41, which is consistent with the negative CoIP results.

Few other F-box proteins have been demonstrated to act independently of the SCF complex to regulate cellular processes. Mdm30 and Mfb1 control mitochondria morphology, Ctf13 is a centromere-binding protein (which also interacts with Skp1) and Fbh1 exhibits helicase activity (Dürr et al., 2006, Kitagawa et al., 1999, Kim et al., 2002, 2004). Aside from Cullin1, Cullin7 has also been reported to build the foundation of an SCF complex (Jin et al., 2004). FBXW8 is the only protein known so far to form Cullin7based SCF complex, but such a complex differs in several aspects from the canonical 
SCF ligase. For example, FBXW8 does not bind to Cullin7 via its F-box domain but utilizes the WD40 domain instead. Moreover, while Cullin 1 binds directly to Skp1, no interaction between Cullin7 and Skp1 can be detected when FBXW8 is not present. Thus, Skp1 is recruited to Cullin7 by FBXW8 and binds to its F-box domain (Dias et al., 2002). Also the mechanism of substrate binding to the Cullin7-FBXW8 complex is not clear. However it has been recently suggested that Cul7/Rbx1/FBXW8 may associate with Cul1/Rbx1/F-box to facilitate the ubiquitination of its substrates (Ponyeam and Hagen, 2012). The interaction may occur between FBXW8 and various F-box proteins of the SCF complex (Ponyeam and Hagen, 2012). Hitherto, only a limited number of substrates have been identified for the Cullin7-based ubiquitin ligase. These include cyclin D1, IRS-1 (insulin receptor substrate 1) and a Golgi protein, GRASP65 (Xu et al., 2008, Okabe et al., 2006, Litterman et al., 2011). Experiments performed by C. Mukherjee demonstrated that FBXO41 constitutes a second known F-box protein interacting with Cullin7.

Having identified a novel Cullin7-based E3 ligase, I was prompted to examine DISC1 and NDEL1 as potential substrates. In case of DISC1, there was neither a change of expression level in the FBXO41 knockout brain, nor did DISC1 interact with Cullin7, indicating that it is not a substrate of FBXO41. However, NDEL1 accumulated in the FBXO41 knockout cerebellum at P5 when the neuronal migration takes place but not at $\mathrm{P} 16$, when the migration is completed. This result suggests that FBXO41 affects the levels of NDEL1 in migrating neurons and is consistent with the fact that overexpression of NDEL1 has been found to impair neuronal migration (Shmueli et al., 2010). Hence, elevated levels of NDEL1 resulting from the FBXO41 downregulation may underlie the migration defects observed upon the knockdown of FBXO41 in rat cerebella and in the FBXO41 knockout mice. Further experiments, including in vitro and in vivo ubiquitination studies would be necessary to determine if FBXO41 regulates the NDEL1 levels in a ligase activity-dependent manner. 


\subsection{FBXO41 is a selective regulator of axon growth}

Since FBXO41 knockout brains did not show any signs of apoptosis, I decided to examine other aspects of neurodevelopment. Strikingly, FBXO41 appears to exclusively stimulate axon but not dendrite growth. Since microtubule polymerization is crucial for axon growth (Tanaka et al., 1995) and the centrosome constitutes the main microtubule nucleation site (Baas et al., 2005b), I investigated if the axon growth-promoting effect of FBXO41 depends on its centrosomal localization. I demonstrated that even though not localized at the centrosome, the FBXO41 $\Delta$ CTR stimulated axon growth as potently as FBXO41 WT, suggesting that the centrosomal localization is dispensable for FBXO41mediated axon growth regulation.

Although the centrosome has been shown to play an important role in axon specification in vitro (Zmuda and Rivas, 1998), a recent study demonstrated that centrosome ablation in isolated rodent hippocampal neurons did not disturb axon extension. Additionally, acentrosomal microtubule nucleation was observed in the ablated neurons (Stiess et al., 2010). Consistent with this finding, FBXO41 may utilize centrosome-independent mechanisms of cytoskeletal regulation in the control of axon growth.

A variety a regulatory pathways is involved in the control of axon growth. DISC1 is one of the important neurite growth-promoting proteins (Kamiya et al., 2005). Since FBXO41 interacts with DISC1, I analyzed if FBXO41 cooperates with DISC1 to induce axon growth. The overexpression experiments demonstrated that the mutant of FBXO41 that does not bind DISC1, still promotes axon growth to the extend of the wild type FBXO41. Thus, DISC1 binding to FBXO41 is dispensable for axon growth regulation. This finding was substantiated by the epistasis analysis, which showed, that the opposite effects of FBXO41 overexpression and DISC1 knockdown neutralize each others phenotype in CGNs. The epistasis experiment demonstrated that DISC1 and FBXO41 act independently to regulate axonal length. Control of axon growth includes for instance the kinase GSK3 with its targets APC, CLASP2, CRMP-2, MAP1B and tau (Zhou and Snider, 2006). Moreover, JNK (c-Jun N-terminal kinase) and MAP kinase pathway have been suggested to play a role in axon elongation (Xiao et al., 2006, Ciani and Salinas, 2007, Markus et al., 2002b). It remains to be elucidated if FBXO41 
participates in one of the aforementioned pathways to promote axon growth.

Analyses of dendrite length and complexity revealed that FBXO41 is a selective regulator of axon but not dendrite growth. Many proteins that control neural development, like DISC1 (Kamiya et al., 2005) or FBXO31 (Vadhvani et al., 2013) are involved in general mechanisms of neurite extension. However, it is also essential that either axon growth or dendrite growth can be controlled independently from each other. Several factors, such as p300-SnoN complex (Ikeuchi et al., 2009) or NeuroD (Gaudillière et al., 2004) act solely on axon or dendrite growth, respectively. Although FBXO41 is localized both in the axon and in dendrites, it restricts its growth-controlling function only to the axon. Since it is not the compartmentalization that grants the spatial selectivity in the case of FBXO41, other mechanisms have to apply. One of the possible explanations is an interaction with an axon-specific protein, that would mediate the growth-promoting effect of FBXO41. This is consistent with the finding of my colleague, C. Mukherjee, who identified NFM (neurofilament medium polypeptide) as an interacting partner of FBXO41. NFM has been implicated in regulation of axon diameter and thus controlling its conduction properties (Friede and Samorajski, 1970, Sakaguchi et al., 1993), but no clear line of evidence on NFM in axon elongation has been published so far. C. Mukherjee demonstrated that NFM knockdown in rat CGNs increases axon length and that FBXO41 and NFM regulate axon growth in a cooperative manner. It remains to be determined if NFM is ubiquitinated by $\mathrm{SCF}^{\mathrm{FBXO} 41}$ or if FBXO41 exerts its axon growth regulation by employing a ubiquitination-independent mechanisms.

Given the cytoplasmic localization of FBXO41 in neurons, it is possible that it controls not only axon growth, but also other cellular processes like neurogenesis, dendritic spine maturation and synaptic function. Interestingly, DISC1 is also involved in the control of neural progenitor proliferation and synaptic transmission. DISC1 promotes neurogenesis by interacting with GSK3 $\beta$. This interaction deactivates GSK3 $\beta$, which reduces the phosphorylation of $\beta$-catenin and stabilizes it (Mao et al., 2009). Furthermore, DISC1 regulates the spine size of the glutamatergic synapses by regulating the activity of Rac1 (Hayashi-Takagi et al., 2010). It requires further investigation if FBXO41 plays a role in neural progenitor proliferation and synaptic function, as well as determining 
if interaction with DISC1 may be important for the regulation of these processes.

\subsection{Perspective: FBXO41 in disease and regeneration}

Disruption of neuronal migration and morphogenesis often leads to neurodevelopmental and neurodegenerative disorders. One group of the neurodevelopmental disorders, congenital ataxias, results mainly from the defects in neural development and is characterized by various malformations of the cerebellar structure as well as of other brain regions and CNS-independent defects. Patients suffering from ataxia display motor dysfunction, muscle hypotonia and impairment of development (Millen and Gleeson, 2008). Anatomical analysis provide a basis for distinguishing different types of ataxias. For instance Joubert syndrome is characterized by defects of the brainstem, cerebellum and the cerebellar peduncles (Maria et al., 1999). In turn, hypoplastic vermis, enlarged fourth ventricle and enlarged posterior skull are the hallmarks of the DandyWalker malformation (Parisi and Dobyns, 2003, Forzano et al., 2007). Given the motor phenotype of the FBXO41 knockout mice, including abnormal gait, disturbed balance and tremors, it is possible that mutations in FBXO41 may cause congenital ataxia.

Lissencephaly or "smooth brain" is one of the best characterized disorders caused by defective migration. It is mainly associated with mutations in two genes, LIS1 (Lissencephaly 1) (Reiner et al., 1993) and DCX (Doublecortin) (Gleeson et al., 1998). The common symptoms of lissencephaly are: smooth brain surface, cerebral cortex consisting of four aberrant layers, hypoplastic cerebellum, intractable epilepsy, hypotonia and mental retardation (Dobyns and Truwit, 1995). LIS1 heterozygous mice exhibit abnormal layering in the cortex, cerebellum, hippocampus and olfactory bulb (Cahana et al., 2001). Furthermore, the heterozygous animals show motor defects and impaired cognition (Paylor et al., 1999). FBXO41 knockout mice share some of these phenotypes, including developmental defects of cerebellum and motor deficits. Therefore, it would be interesting to examine the lamination of other brain areas of FBXO41 knockout and heterozygous mice. Behavioral tests could also provide more insight into the cognitive abilities of the FBXO41-deficient animals. Additionally, several of the genes implicated 
in lissencephaly regulate not only neuronal migration but also axon outgrowth (Kara et al., 2010). In concomitance with that, the pathology of lissencephaly shows abnormalities in axon growth and guidance (Grabham et al., 2007, Deuel et al., 2006). Disruption of axon growth leads to white matter deformations, agenesis of the corpus callosum and enlarged ventricles in the lissencephaly patients. The resulting failure to establish proper connectivity in the brain may cause the seizure symptoms (Liu, 2011). Abnormal axonal tracts are also one of the hallmarks of subcortical band heterotopia, which affects women with mutations in the DCX gene (Gleeson et al., 1999). Hence, while the axon growth of isolated FBXO41 knockout neurons is significantly impaired, an in depth analysis of the main axonal tracts in the FBXO41 knockout mouse may provide an insight on whether or not FBXO41 deficit results in the connectivity disruption, similar to that observed in the lissencephaly patients.

Also mutations in genes, whose products regulate axon growth and migration have been associated with several neurodegenerative diseases. For instance mutations in the PFN1 (profilin 1) gene have been shown to cause familiar amyotrophic lateral sclerosis (FALS) (Wu et al., 2012a). PFN1 regulates actin polymerization and overexpression of its ALS-linked mutants inhibits axon growth (Wu et al., 2012a). Moreover, mutations in the SMN1 (survival motoneuron 1) gene are associated with spinal muscular atrophy (SMA). SMN1 deficit in mouse motor neurons causes reduction in axonal, but not dendritic length and its overexpression in PC12 cells promotes the growth of neurites (Rossoll et al., 2003). Since both diseases are characterized by motor neuron loss, it would be important to assess the survival of motoneurons in FBXO41 knockout mice.

The stimulation of axon growth is a major problem in the CNS after injury. While peripheral neurons have a substantial regenerative potential, neurons of the CNS fail to regrow axons. Various gene therapy approaches have been applied to increase the regenerative abilities of injured neurons. Most of them include local delivery of growth factors, but it has been shown that simultaneous stimulation of intracellular signaling improves the outcome of the therapy (Hollis II et al., 2009), making FBXO41 an interesting clinical target. However, while my study demonstrates that FBXO41 promotes axon growth in the developing neurons, the mechanisms of axon regeneration may 
differ from the ones in the initial axon development (Blizzard et al., 2007). Additionally, the mature CNS constitutes an unfavorable environment for axon regeneration, which can be attributed to several factors. First of all, myelin proteins like NOGO, MAG (myelin-associated glycoprotein), and Omgp (oligodendrocyte-myelin glycoprotein) (Xie and Zheng, 2008) as well as the lipid sulfatide (Winzeler et al., 2011) are known inhibitors of axon growth. Furthermore, the growth factor deficit (Tuszynski, M.H., $\mathrm{Lu}, 2008$, Widenfalk et al., 2001) and the presence of repulsive guidance cues such as ephrins, semaphorins and netrins (Benson et al., 2005, Bolsover et al., 2008, Löw et al., 2008, Niclou et al., 2003) contribute to the inhibition of nerve regeneration. Finally, axon growth is also restricted by the glial scar (Fitch and Silver, 2008, Silver and Miller, 2004), inflammatory processes (Popovich and McTigue, 2009) and components of the extracellular matrix, like chondroitin-sulfateproteoglycans (Silver and Miller, 2004). Thus, additional in vitro and in vivo injury studies could shed more light on the regenerative potential of FBXO41 overexpression. Axon growth assays using myelin coated plates and evaluation of FBXO41 effect upon optic nerve crush or in spinal cord injury models could provide more insight into its role in the regeneration processes.

\subsection{Conclusion}

My study identified the F-box protein FBXO41 as a novel neuron-specific protein in the CNS. I found that FBXO41 is a crucial regulator of cerebellar development (Fig. 4.1). FBXO41 acts together with DISC1 at the centrosome to promote neuronal migration of CGNs in the cerebellar cortex. Also, DISC1 bridges FBXO41's association with NDEL1, a known regulator of neuronal migration. Moreover, FBXO41 may affect the expression levels of NDEL1, which is crucial for correct migration. In addition, I demonstrated that although the interaction of FBXO41 and DISC1 is required for proper neuronal migration, this interaction is dispensable for FBXO41's role in axon growth regulation of CGNs. Finally, the generation of an FBXO41 knockout mouse enabled to confirm the neurodevelopmental role of FBXO41 in the cerebellum. Taken together, my study constitutes an important step towards understanding the molecular pathways that regulate 
the development of the cerebellum. It also contributes to a better understanding of the functions of F-box proteins in this process. Ultimately, my results may establish a basis for the identification of new causative genes for neurodevelopmental disorders such as congenital ataxias and provide a source of potential therapeutic approaches.

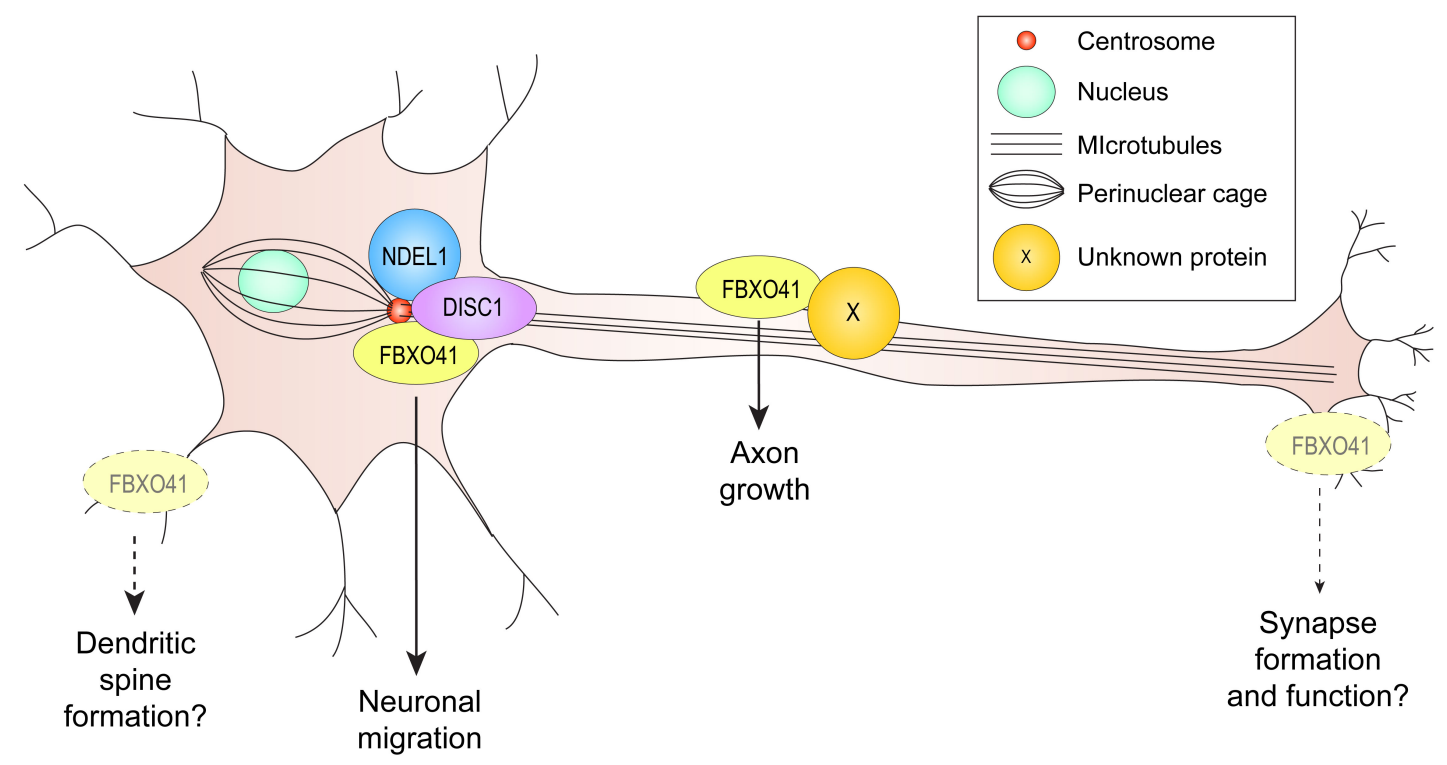

Figure 4.1.: FBXO41 is a multifunctional protein in neurons. FBXO41 is localized to the centrosome and to the cytoplasm, including axons and dendrites. FBXO41's function in neurons is tightly connected to its localization and likely determined by its distinct interacting partners. Centrosomal FBXO41 regulates neuronal migration and the interaction with DISC1 is indispensable for this process. Moreover, my study demonstrated that FBXO41 forms a complex with NDEL1 in the presence of DISC1, and this interaction may be crucial for assuring of proper expression levels of NDEL1. On the other hand, cytoplasmic FBXO41 acts as a positive regulator of axon growth. Interaction with axonal proteins like NFM may mediate the function of FBXO41 in axon growth control. Additionally, cytoplasmic localization of FBXO41, may indicate its function in dendritic spine development or synaptic function, which remains to be determined. Dashed lines indicate putative functions of FBXO41. 



\section{Bibliography}

Aghajan, M., Jonai, N., Flick, K., Fu, F., Luo, M., Cai, X., Ouni, I., Pierce, N., Tang, X., Lomenick, B., Damoiseaux, R., Hao, R., del Moral, P. M., Verma, R., Li, Y., Li, C., Houk, K. N., Jung, M. E., Zheng, N., Huang, L., Deshaies, R. J., Kaiser, P., and Huang, J. (2010). Chemical genetics screen for enhancers of rapamycin identifies a specific inhibitor of an SCF family E3 ubiquitin ligase. Nat Biotech, 28(7):738-742.

Alcantara, S., Ruiz, M., De Castro, F., Soriano, E., and Sotelo, C. (2000). Netrin 1 acts as an attractive or as a repulsive cue for distinct migrating neurons during the development of the cerebellar system. Development, 127(7):1359-1372.

Altman, J. (1972). Postnatal development of the cerebellar cortex in the rat. I. The external germinal layer and the transitional molecular layer. The fournal of Comparative Neurology, 145(3):353-397.

Altman, J. and Bayer, S. A. (1997). Development of the Cerebellar System: In Relation to Its Evolution, Structure, and Functions. CRC Press, 1 edition.

Alvarez-Buylla, A. (1997). Mechanism of migration of olfactory bulb interneurons. Seminars in Cell \& Developmental Biology, 8(2):207-213.

Anderson, S. A., Eisenstat, D. D., Shi, L., and Rubenstein, J. L. R. (1997). Interneuron Migration from Basal Forebrain to Neocortex: Dependence on Dlx Genes. Science, 278(5337):474-476.

Arakawa, Y., Bito, H., Furuyashiki, T., Tsuji, T., Takemoto-Kimura, S., Kimura, K., Nozaki, K., Hashimoto, N., and Narumiya, S. (2003). Control of axon elongation via 
an SDF- $1 \alpha /$ Rho/mDia pathway in cultured cerebellar granule neurons. The fournal of Cell Biology, 161(2):381-391.

Arguello, P. A. and Gogos, J. a. (2006). Modeling madness in mice: one piece at a time. Neuron, 52(1):179-96.

Arlotta, P., Molyneaux, B. J., Chen, J., Inoue, J., Kominami, R., and Macklis, J. D. (2005). Neuronal Subtype-Specific Genes that Control Corticospinal Motor Neuron Development In Vivo. Neuron, 45(2):207-221.

Arnaud, L., Ballif, B. A., and Cooper, J. A. (2003a). Regulation of Protein Tyrosine Kinase Signaling by Substrate Degradation during Brain Development. Molecular and Cellular Biology, 23(24):9293-9302.

Arnaud, L., Ballif, B. A., Förster, E., and Cooper, J. A. (2003b). Fyn Tyrosine Kinase Is a Critical Regulator of Disabled-1 during Brain Development. Current Biology, 13(1):917.

Austin, C. P., Ky, B., Ma, L., Morris, J. A., and Shughrue, P. J. (2004). Expression of disrupted-in-schizophrenia-1, a schizophrenia-associated gene, is prominent in the mouse hippocampus throughout brain development. Neuroscience, 124(1):3-10.

Baas, P. W., Karabay, A., and Qiang, L. (2005a). Microtubules cut and run. Trends in Cell Biology, 15(10):518-524.

Baas, P. W., Karabay, A., and Qiang, L. (2005b). Microtubules cut and run. Trends in Cell Biology, 15(10):518-524.

Bai, C., Sen, P., Hofmann, K., Ma, L., Goebl, M., Harper, J., and Elledge, S. J. (1996). SKP1 Connects Cell Cycle Regulators to the Ubiquitin Proteolysis Machinery through a Novel Motif, the F-Box. Cell, 86(2):263-274.

Bai, J., Ramos, R. L., Ackman, J. B., Thomas, A. M., Lee, R. V., and LoTurco, J. J. (2003). RNAi reveals doublecortin is required for radial migration in rat neocortex. Nat Neurosci, 6(12):1277-1283. 
Barnes, A. P. and Polleux, F. (2009). Establishment of axon-dendrite polarity in developing neurons. Annual review of neuroscience, 32:347-81.

Bartlett, P. and Banker, A. (1984). An electron microscopic study of the development of axons and dendrites by hippocampal neurons in culture. II. Synaptic relationships. The fournal of neuroscience: the official journal of the Society for Neuroscience, 4(8):19541965.

Bartlett, P., Banker, A., Easton, S., Bartlett, W. P., and Banker, G. A. (1984). An electron microscopic study of the development of axons and dendrites by hippocampal neurons in culture. I. Cells which develop without intercellular contacts. The fournal of Neuroscience, 4(8):1944-1953.

Behar, T. N. (1997). Neurotrophins stimulate chemotaxis of embryonic cortical neurons. Eur. F. Neurosci., 9:2561-2570.

Behrends, C. and Harper, J. W. (2011). Constructing and decoding unconventional ubiquitin chains. Nat Struct Mol Biol, 18(5):520-528.

Bellion, A., Baudoin, J.-P., Alvarez, C., Bornens, M., and Métin, C. (2005). Nucleokinesis in Tangentially Migrating Neurons Comprises Two Alternating Phases: Forward Migration of the Golgi/Centrosome Associated with Centrosome Splitting and Myosin Contraction at the Rear. The fournal of Neuroscience, 25(24):5691-5699.

Benson, M. D., Romero, M. I., Lush, M. E., Lu, Q. R., Henkemeyer, M., and Parada, L. F. (2005). Ephrin-B3 is a myelin-based inhibitor of neurite outgrowth. Proceedings of the National Academy of Sciences of the United States of America, 102(30):10694-10699.

Bentley, D. and O’Connor, T. P. (1994). Cytoskeletal events in growth cone steering. Current Opinion in Neurobiology, 4(1):43-48.

Bibel, M. and Barde, Y.-A. (2000). Neurotrophins: key regulators of cell fate and cell shape in the vertebrate nervous system. Genes \& Development, 14(23):2919-2937. 
Bielas, S., Higginbotham, H., Koizumi, H., Tanaka, T., and Gleeson, J. G. (2004). Cortical neuronal migration mutants suggest separate but intersecting pathways. Annual review of cell and developmental biology, 20:593-618.

Blizzard, C. a., Haas, M. a., Vickers, J. C., and Dickson, T. C. (2007). Cellular dynamics underlying regeneration of damaged axons differs from initial axon development. The European journal of neuroscience, 26(5):1100-8.

Bock, H. H. and Herz, J. (2003). Reelin Activates Src Family Tyrosine Kinases in Neurons. Current Biology, 13(1):18-26.

Boix-Perales, H., Horan, I., Wise, H., Lin, H.-R., Chuang, L.-C., Yew, P. R., and Philpott, A. (2007). The E3 ubiquitin ligase skp2 regulates neural differentiation independent from the cell cycle. Neural Development, 2(1):27.

Bolsover, S., Fabes, J., and Anderson, P. N. (2008). Axonal guidance molecules and the failure of axonal regeneration in the adult mammalian spinal cord. Restorative Neurology and Neuroscience, 26(2):117-130.

Bord, L., Wheeler, J., Paek, M., Saleh, M., Lyons-Warren, A., Ross, C. A., Sawamura, N., and Sawa, A. (2006). Primate disrupted-in-schizophrenia-1 (DISC1): High divergence of a gene for major mental illnesses in recent evolutionary history. Neuroscience Research, 56(3):286-293.

Bradshaw, N. J., Soares, D. C., Carlyle, B. C., Ogawa, F., Davidson-Smith, H., Christie, S., Mackie, S., Thomson, P. A., Porteous, D. J., and Millar, J. K. (2011). PKA Phosphorylation of NDE1 Is DISC1/PDE4 Dependent and Modulates Its Interaction with LIS1 and NDEL1. The fournal of Neuroscience, 31(24):9043-9054.

Brandon, N. J., Handford, E. J., Schurov, I., Rain, J.-C., Pelling, M., Duran-Jimeniz, B., Camargo, L. M., Oliver, K. R., Beher, D., Shearman, M. S., and Whiting, P. J. (2004). Disrupted in Schizophrenia 1 and Nudel form a neurodevelopmentally regulated protein complex: implications for schizophrenia and other major neurological disorders. Molecular and cellular neurosciences, 25(1):42-55. 
Brandon, N. J., Schurov, I., Camargo, L. M., Handford, E. J., Duran-Jimeniz, B., Hunt, P., Millar, J. K., Porteous, D. J., Shearman, M. S., and Whiting, P. J. (2005). Subcellular targeting of DISC1 is dependent on a domain independent from the Nudel binding site. Molecular and Cellular Neuroscience, 28(4):613-624.

Bridgman, P. C. and Dailey, M. E. (1989). The organization of myosin and actin in rapid frozen nerve growth cones. The fournal of Cell Biology, 108(1):95-109.

Brose, K. and Tessier-Lavigne, M. (2000). Slit proteins: key regulators of axon guidance, axonal branching, and cell migration. Curr. Opin. Neurobiol., 10:95-102.

Buck, K. B. and Zheng, J. Q. (2002). Growth Cone Turning Induced by Direct Local Modification of Microtubule Dynamics. The fournal of Neuroscience, 22(21):9358-9367.

Butler, S. J. and Dodd, J. (2003). A Role for BMP Heterodimers in Roof Plate-Mediated Repulsion of Commissural Axons. Neuron, 38(3):389-401.

Caceres, A., Banker, G., Steward, O., Binder, L., and Payne, M. (1984). MAP2 is localized to the dendrites of hippocampal neurons which develop in culture. Developmental Brain Research, 13(2):314-318.

Cadwell, K. and Coscoy, L. (2005). Ubiquitination on Nonlysine Residues by a Viral E3 Ubiquitin Ligase. Science, 309(5731):127-130.

Cahana, A., Escamez, T., Nowakowski, R. S., Hayes, N. L., Giacobini, M., von Holst, A., Shmueli, O., Sapir, T., McConnell, S. K., Wurst, W., Martinez, S., and Reiner, O. (2001). Targeted mutagenesis of Lis1 disrupts cortical development and LIS1 homodimerization. Proceedings of the National Academy of Sciences, 98(11):6429-6434.

Camargo, A. C. M., Caldo, H., and Emson, P. C. (1983). Degradation of neurotensin by rabbit brain endo-oligopeptidase A and endo-oligopeptidase B (prolineendopeptidase). Biochemical and Biophysical Research Communications, 116(3):11511159. 
Camargo, L. M., Collura, V., Rain, J.-C., Mizuguchi, K., Hermjakob, H., Kerrien, S., Bonnert, T. P., Whiting, P. J., and Brandon, N. J. (2007). Disrupted in Schizophrenia 1 Interactome: evidence for the close connectivity of risk genes and a potential synaptic basis for schizophrenia. Molecular psychiatry, 12(1):74-86.

Chai, X., Förster, E., Zhao, S., Bock, H. H., and Frotscher, M. (2009). Reelin Stabilizes the Actin Cytoskeleton of Neuronal Processes by Inducing n-Cofilin Phosphorylation at Serine3. The fournal of Neuroscience, 29(1):288-299.

Charron, F., Stein, E., Jeong, J., McMahon, A. P., and Tessier-Lavigne, M. (2003). The Morphogen Sonic Hedgehog Is an Axonal Chemoattractant that Collaborates with Netrin-1 in Midline Axon Guidance. Cell, 113(1):11-23.

Chau, V., Tobias, J. W., Bachmair, A., Marriotr, D., Ecker, D. J., Gonda, D. K., Varshavsky, A., and Marriott, D. (1989). A multiubiquitin chain is confined to specific lysine in a targeted short-lived protein. Science, 243(4898):1576-1583.

Chen, Z. J., Parent, L., and Maniatis, T. (1996). Site-specific phosphorylation of IkappaBalpha by a novel ubiquitination-dependent protein kinase activity. Cell, 84(6):85362.

Chhabra, E. S. and Higgs, H. N. (2007). The many faces of actin: matching assembly factors with cellular structures. Nature cell biology, 9(10):1110-21.

Ciani, L. and Salinas, P. C. (2007). c-Jun N-terminal kinase (JNK) cooperates with Gsk3beta to regulate Dishevelled-mediated microtubule stability. BMC cell biology, $8: 27$.

Ciechanover, A., Heller, H., Katz-Etzion, R., and Hershko, A. (1981). Activation of the heat-stable polypeptide of the ATP-dependent proteolytic system. Proceedings of the National Academy of Sciences, 78(2):761-765.

Clapcote, S. J., Lipina, T. V., Millar, J. K., Mackie, S., Christie, S., Ogawa, F., Lerch, J. P., Trimble, K., Uchiyama, M., Sakuraba, Y., Kaneda, H., Shiroishi, T., Houslay, M. D., 
Henkelman, R. M., Sled, J. G., Gondo, Y., Porteous, D. J., and Roder, J. C. (2007). Behavioral phenotypes of Disc1 missense mutations in mice. Neuron, 54(3):387-402.

Csernansky, J. G., Wang, L., Jones, D., Rastogi-Cruz, D., Posener, J. a., Heydebrand, G., Miller, J. P., and Miller, M. I. (2002). Hippocampal deformities in schizophrenia characterized by high dimensional brain mapping. The American journal of psychiatry, 159(12):2000-6.

Dammermann, A. and Merdes, A. (2002). Assembly of centrosomal proteins and microtubule organization depends on PCM-1. The fournal of Cell Biology, 159(2):255-266.

D’Arcangelo, G., G. Miao, G., Chen, S.-C., Scares, H. D., Morgan, J. I., and Curran, T. (1995). A protein related to extracellular matrix proteins deleted in the mouse mutant reeler. Nature, 374(6524):719-723.

Davis, L., Banker, G. A., and Steward, O. (1987). Selective dendritic transport of RNA in hippocampal neurons in culture. Nature, 330(6147):477-479.

de Anda, F. C., Meletis, K., Ge, X., Rei, D., and Tsai, L.-H. (2010). Centrosome Motility Is Essential for Initial Axon Formation in the Neocortex. The fournal of Neuroscience, 30(31):10391-10406.

de Wit, M. C. Y., Kros, J. M., Halley, D. J. J., de Coo, I. F. M., Verdijk, R., Jacobs, B. C., and Mancini, G. M. S. (2009). Filamin A mutation, a common cause for periventricular heterotopia, aneurysms and cardiac defects. Journal of Neurology, Neurosurgery \& Psychiatry, 80(4):426-428.

Dehmelt, L. and Halpain, S. (2004). Actin and microtubules in neurite initiation: are MAPs the missing link? fournal of neurobiology, 58(1):18-33.

Dent, E. W. and Gertler, F. B. (2003). Cytoskeletal dynamics and transport in growth cone motility and axon guidance. Neuron, 40(2):209-27.

Deshaies, R. J. (1999). SCF AND CULLIN/RING H2-BASED UBIQUITIN LIGASES. Annual Review of Cell and Developmental Biology, 15(1):435-467. 
Deshaies, R. J. and Joazeiro, C. A. P. (2009). RING Domain E3 Ubiquitin Ligases. Annual Review of Biochemistry, 78(1):399-434.

Deuel, T. A. S., Liu, J. S., Corbo, J. C., Yoo, S.-Y., Rorke-Adams, L. B., and Walsh, C. A. (2006). Genetic Interactions between Doublecortin and Doublecortin-like Kinase in Neuronal Migration and Axon Outgrowth. Neuron, 49(1):41-53.

Dias, D. C., Dolios, G., Wang, R., and Pan, Z.-Q. (2002). CUL7: A DOC domaincontaining cullin selectively binds Skp1.Fbx29 to form an SCF-like complex. Proceedings of the National Academy of Sciences of the United States of America, 99(26):16601-6.

Dijkhuizen, P. A. and Ghosh, A. (2005). Regulation of dendritic growth by calcium and neurotrophin signaling. In J. van Pelt C.N. Levelt, A. van Ooyen, G.J.A. Ramakers and P.R. Roelfsema BT - Progress in Brain Research, M. K., editor, Development, Dynamics and Pathiology of Neuronal Networks: from Molecules to Functional Circuits, volume Volume 147, pages 15-27. Elsevier.

DiTella, M. C., Feiguin, F., Carri, N., Kosik, K. S., and Caceres, A. (1996). MAP-1B/TAU functional redundancy during laminin-enhanced axonal growth. Fournal of Cell Science, 109(2):467-477.

Dobyns, W. B. and Truwit, C. L. (1995). Lissencephaly and Other Malformations of Cortical Development: 1995 Update. Neuropediatrics, 26(03):132-147.

Dotti, C. G., Sullivan, C. A., and Banker, G. A. (1988). The establishment of polarity by hippocampal neurons in culture. The fournal of Neuroscience, 8(4):1454-1468.

Duan, X., Chang, J. H., Ge, S., Faulkner, R. L., Kim, J. Y., Kitabatake, Y., Liu, X.-b., Yang, C.-H., Jordan, J. D., Ma, D. K., Liu, C. Y., Ganesan, S., Cheng, H.-J., Ming, G.-1., Lu, B., and Song, H. (2007). Disrupted-In-Schizophrenia 1 regulates integration of newly generated neurons in the adult brain. Cell, 130(6):1146-58.

Dürr, M., Escobar-Henriques, M., Merz, S., Geimer, S., Langer, T., and Westermann, B. (2006). Nonredundant Roles of Mitochondria-associated F-Box Proteins Mfb1 and 
Mdm30 in Maintenance of Mitochondrial Morphology in Yeast. Molecular Biology of the Cell, 17(9):3745-3755.

Eastwood, S. L., Hodgkinson, C. A., and Harrison, P. J. (2009). DISC-1 Leu607Phe alleles differentially affect centrosomal PCM1 localization and neurotransmitter release. $\mathrm{Mol}$ Psychiatry, 14(6):556-557.

Eastwood, S. L., Walker, M., Hyde, T. M., Kleinman, J. E., and Harrison, P. J. (2010). The DISC1 Ser704Cys substitution affects centrosomal localization of its binding partner PCM1 in glia in human brain. Human Molecular Genetics, 19(12):2487-2496.

Edmondson, J. C. and Hatten, M. E. (1987). Glial-guided granule neuron migration in vitro: a high-resolution time- lapse video microscopic study. The fournal of Neuroscience, 7(6):1928-1934.

Ekelund, J., Hennah, W., Hiekkalinna, T., Parker, A., Meyer, J., Lonnqvist, J., and Peltonen, L. (2004). Replication of 1q42 linkage in Finnish schizophrenia pedigrees. Mol Psychiatry, 9(11):1037-1041.

Ekelund, J., Hovatta, I., Parker, a., Paunio, T., Varilo, T., Martin, R., Suhonen, J., Ellonen, P., Chan, G., Sinsheimer, J. S., Sobel, E., Juvonen, H., Arajärvi, R., Partonen, T., Suvisaari, J., Lönnqvist, J., Meyer, J., and Peltonen, L. (2001). Chromosome 1 loci in Finnish schizophrenia families. Human molecular genetics, 10(15):1611-7.

Ekelund, J., Lichtermann, D., Hovatta, I., Ellonen, P., Suvisaari, J., Terwilliger, J. D., Juvonen, H., Varilo, T., Arajärvi, R., Kokko-Sahin, M.-L., Lönnqvist, J., and Peltonen, L. (2000). Genome-wide scan for schizophrenia in the Finnish population: evidence for a locus on chromosome 7q22. Human Molecular Genetics, 9(7):1049-1057.

Enomoto, A., Asai, N., Namba, T., Wang, Y., Kato, T., Tanaka, M., Tatsumi, H., Taya, S., Tsuboi, D., Kuroda, K., Kaneko, N., Sawamoto, K., Miyamoto, R., Jijiwa, M., Murakumo, Y., Sokabe, M., Seki, T., Kaibuchi, K., and Takahashi, M. (2009). Roles of Disrupted-In-Schizophrenia 1-Interacting Protein Girdin in Postnatal Development of the Dentate Gyrus. Neuron, 63(6):774-787. 
Feldman, R. M., Correll, C. C., Kaplan, K. B., and Deshaies, R. J. (1997). A complex of Cdc4p, Skp1p, and Cdc53p/cullin catalyzes ubiquitination of the phosphorylated CDK inhibitor Sic1p. Cell, 91(2):221-30.

Feng, L., Allen, N. S., Simo, S., and Cooper, J. A. (2007). Cullin 5 regulates Dab1 protein levels and neuron positioning during cortical development. Genes \& Development, 21(21):2717-2730.

Feng, Y., Olson, E. C., Stukenberg, P., Flanagan, L. A., Kirschner, M. W., and Walsh, C. A. (2000). LIS1 Regulates CNS Lamination by Interacting with mNudE, a Central Component of the Centrosome. Neuron, 28(3):665-679.

Fitch, M. T. and Silver, J. (2008). CNS injury, glial scars, and inflammation: Inhibitory extracellular matrices and regeneration failure. Experimental Neurology, 209(2):294301.

Flames, N., Long, J. E., Garratt, A. N., Fischer, T. M., Gassmann, M., Birchmeier, C., Lai, C., Rubenstein, J. L. R., and Marín, O. (2004). Short- and Long-Range Attraction of Cortical GABAergic Interneurons by Neuregulin-1. Neuron, 44(2):251-261.

Fonzo, A. D., Dekker, M. C. J., Montagna, P., Baruzzi, A., Yonova, E. H., Guedes, L. C., Szczerbinska, A., Zhao, T., Dubbel-Hulsman, L. O. M., Wouters, C. H., de Graaff, E., Oyen, W. J. G., Simons, E. J., Breedveld, G. J., Oostra, B. A., Horstink, M. W., and Bonifati, V. (2009). FBXO7 mutations cause autosomal recessive, early-onset parkinsonianpyramidal syndrome. Neurology, 72(3):240-245.

Forzano, F., Mansour, S., Ierullo, A., Homfray, T., and Thilaganathan, B. (2007). Posterior fossa malformation in fetuses: a report of 56 further cases and a review of the literature. Prenatal Diagnosis, 27(6):495-501.

Fox, J. W., Lamperti, E. D., Ekşioğlu, Y. Z., Hong, S. E., Feng, Y., Graham, D. A., Scheffer, I. E., Dobyns, W. B., Hirsch, B. A., Radtke, R. A., Berkovic, S. F., Huttenlocher, P. R., and Walsh, C. A. (1998). Mutations in filamin 1 Prevent Migration of Cerebral Cortical Neurons in Human Periventricular Heterotopia. Neuron, 21(6):1315-1325. 
Frescas, D. and Pagano, M. (2008). Deregulated proteolysis by the F-box proteins SKP2 and [beta]-TrCP: tipping the scales of cancer. Nat Rev Cancer, 8(6):438-449.

Friede, R. L. and Samorajski, T. (1970). Axon caliber related to neurofilaments and microtubules in sciatic nerve fibers of rats and mice. The Anatomical Record, 167(4):379387.

Friston, K. J., Liddle, P. F., Frith, C. D., Hirsch, S. R., and Frackowiak, R. S. J. (1992). THE LEFT MEDIAL TEMPORAL REGION AND SCHIZOPHRENIA: A PET STUDY. Brain, 115(2):367-382.

Fukuda, T., Sugita, S., Inatome, R., and Yanagi, S. (2010). CAMDI, a Novel Disrupted in Schizophrenia 1 (DISC1)-binding Protein, Is Required for Radial Migration. Journal of Biological Chemistry, 285(52):40554-40561.

Fukuda, T., Tokunaga, A., Sakamoto, R., and Yoshida, N. (2011). Fbxl10/Kdm2b deficiency accelerates neural progenitor cell death and leads to exencephaly. Molecular and Cellular Neuroscience, 46(3):614-624.

Galan, J. M. and Haguenauer-Tsapis, R. (1997). Ubiquitin lys63 is involved in ubiquitination of a yeast plasma membrane protein. The EMBO journal, 16(19):5847-54.

Gaudillière, B., Konishi, Y., de la Iglesia, N., Yao, G.-l., and Bonni, A. (2004). A CaMKIINeuroD Signaling Pathway Specifies Dendritic Morphogenesis. Neuron, 41(2):229241.

Ge, X., Frank, C. L., Calderon de Anda, F., and Tsai, L.-H. (2010). Hook3 Interacts with PCM1 to Regulate Pericentriolar Material Assembly and the Timing of Neurogenesis. Neuron, 65(2):191-203.

Gelfand, V. I. and Bershadsky, A. D. (1991). Microtubule Dynamics: Mechanism, Regulation, and Function. Annual Review of Cell Biology, 7(1):93-116.

Gleeson, J. G. (2001). Neuronal migration disorders. Mental Retardation and Developmental Disabilities Research Reviews, 7(3):167-171. 
Gleeson, J. G., Allen, K. M., Fox, J. W., Lamperti, E. D., Berkovic, S., Scheffer, I., Cooper, E. C., Dobyns, W. B., Minnerath, S. R., Ross, M., and Walsh, C. A. (1998). doublecortin, a Brain-Specific Gene Mutated in Human X-Linked Lissencephaly and Double Cortex Syndrome, Encodes a Putative Signaling Protein. Cell, 92(1):63-72.

Gleeson, J. G., Minnerath, S. R., Fox, J. W., Allen, K. M., Luo, R. F., Hong, S. E., Berg, M. J., Kuzniecky, R., Reitnauer, P. J., Borgatti, R., Puche Mira, A., Guerrini, R., Holmes, G. L., Rooney, C. M., Berkovic, S., Scheffer, I., Cooper, E. C., Ricci, S., Cusmai, R., Crawford, T. O., Leroy, R., Andermann, E., Wheless, J. W., Dobyns, W. B., Ross, M. E., and Walsh, C. A. (1999). Characterization of mutations in the gene doublecortin in patients with double cortex syndrome. Annals of Neurology, 45(2):146-153.

Glenn, K. A., Nelson, R. F., Wen, H. M., Mallinger, A. J., and Paulson, H. L. (2008). Diversity in Tissue Expression, Substrate Binding, and SCF Complex Formation for a Lectin Family of Ubiquitin Ligases. Journal of Biological Chemistry, 283(19):12717-12729.

Goldberg, D. J. and Burmeister, D. W. (1986). Stages in axon formation: observations of growth of Aplysia axons in culture using video-enhanced contrast-differential interference contrast microscopy. The fournal of Cell Biology, 103(5):1921-1931.

Goley, E. D. and Welch, M. D. (2006). The ARP2/3 complex: an actin nucleator comes of age. Nat Rev Mol Cell Biol, 7(10):713-726.

Gong, B., Chen, F., Pan, Y., Arrieta-Cruz, I., Yoshida, Y., Haroutunian, V., and Pasinetti, G. M. (2010). SCFFbx2-E3-ligase-mediated degradation of BACE1 attenuates Alzheimer's disease amyloidosis and improves synaptic function. Aging Cell, 9(6):1018-1031.

Gonzalez-Billault, C., Jimenez-Mateos, E. M., Caceres, A., Diaz-Nido, J., Wandosell, F., and Avila, J. (2004). Microtubule-associated protein 1B function during normal development, regeneration, and pathological conditions in the nervous system. fournal of Neurobiology, 58(1):48-59. 
Gonzalez-Billault, C., Owen, R., Gordon-Weeks, P. R., and Avila, J. (2002). Microtubuleassociated protein $1 \mathrm{~B}$ is involved in the initial stages of axonogenesis in peripheral nervous system cultured neurons. Brain Research, 943(1):56-67.

Govek, E.-E., Hatten, M. E., and Van Aelst, L. (2011). The role of Rho GTPase proteins in CNS neuronal migration. Developmental Neurobiology, 71(6):528-553.

Govek, E.-E., Newey, S. E., and Van Aelst, L. (2005). The role of the Rho GTPases in neuronal development. Genes \& Development, 19(1):1-49.

Grabham, P. W., Seale, G. E., Bennecib, M., Goldberg, D. J., and Vallee, R. B. (2007). Cytoplasmic Dynein and LIS1 Are Required for Microtubule Advance during Growth Cone Remodeling and Fast Axonal Outgrowth. The fournal of Neuroscience, 27(21):58235834.

Griffith, E., Walker, S., Martin, C.-A., Vagnarelli, P., Stiff, T., Vernay, B., Sanna, N. A., Saggar, A., Hamel, B., Earnshaw, W. C., Jeggo, P. A., Jackson, A. P., and O’Driscoll, M. (2008). Mutations in pericentrin cause Seckel syndrome with defective ATRdependent DNA damage signaling. Nat Genet, 40(2):232-236.

Guo, J., Yang, Z., Song, W., Chen, Q., Wang, F., Zhang, Q., and Zhu, X. (2006). Nudel Contributes to Microtubule Anchoring at the Mother Centriole and Is Involved in Both Dynein-dependent and -independent Centrosomal Protein Assembly. Molecular Biology of the Cell, 17(2):680-689.

Haas, A. L., Warms, J. V., Hershko, A., and Rose, I. A. (1982). Ubiquitin-activating enzyme. Mechanism and role in protein-ubiquitin conjugation. Fournal of Biological Chemistry, 257(5):2543-2548.

Hamshere, M., Bennett, P., Williams, N., and Al, E. (2005). Genomewide linkage scan in schizoaffective disorder: Significant evidence for linkage at 1q42 close to disc1, and suggestive evidence at 22q11 and 19p13. Archives of General Psychiatry, 62(10):10811088. 
Harada, A., Teng, J., Takei, Y., Oguchi, K., and Hirokawa, N. (2002). MAP2 is required for dendrite elongation, PKA anchoring in dendrites, and proper PKA signal transduction. The fournal of Cell Biology, 158(3):541-549.

Hatten, M. E. (2002). New directions in neuronal migration. Science (New York, N.Y.), 297(5587):1660-3.

Hattori, T., Baba, K., Matsuzaki, S., Honda, A., Miyoshi, K., Inoue, K., Taniguchi, M., Hashimoto, H., Shintani, N., Baba, A., Shimizu, S., Yukioka, F., Kumamoto, N., Yamaguchi, A., Tohyama, M., and Katayama, T. (2007). A novel DISC1-interacting partner DISC1-Binding Zinc-finger protein: implication in the modulation of DISC1dependent neurite outgrowth. Mol Psychiatry, 12(4):398-407.

Hayashi, M. A. F., Portaro, F. C. V., Bastos, M. F., Guerreiro, J. R., Oliveira, V., Gorrão, S. S., Tambourgi, D. V., Sant'Anna, O. A., Whiting, P. J., Camargo, L. M., Konno, K., Brandon, N. J., and Camargo, A. C. M. (2005). Inhibition of NUDEL (nuclear distribution element-like)-oligopeptidase activity by disrupted-in-schizophrenia 1. Proceedings of the National Academy of Sciences of the United States of America, 102(10):38283833.

Hayashi-Takagi, A., Takaki, M., Graziane, N., Seshadri, S., Murdoch, H., Dunlop, A. J., Makino, Y., Seshadri, A. J., Ishizuka, K., Srivastava, D. P., Xie, Z., Baraban, J. M., Houslay, M. D., Tomoda, T., Brandon, N. J., Kamiya, A., Yan, Z., Penzes, P., and Sawa, A. (2010). Disrupted-in-Schizophrenia 1 (DISC1) regulates spines of the glutamate synapse via Rac1. Nat Neurosci, 13(3):327-332.

Heng, Y. H. E., Barry, G., Richards, L. J., and Piper, M. (2012). Nuclear factor I genes regulate neuronal migration. Neuro-Signals, 20(3):159-67.

Hennah, W., Varilo, T., Kestilä, M., Paunio, T., Arajärvi, R., Haukka, J., Parker, A., Martin, R., Levitzky, S., Partonen, T., Meyer, J., Lönnqvist, J., Peltonen, L., and Ekelund, J. (2003). Haplotype transmission analysis provides evidence of association for DISC1 to schizophrenia and suggests sex-dependent effects. Human molecular genetics, 12(23):3151-9. 
Hershko, A., Ciechanover, A., and Rose, I. A. (1981). Identification of the active amino acid residue of the polypeptide of ATP-dependent protein breakdown. Fournal of Biological Chemistry, 256(4):1525-1528.

Hershko, A., Heller, H., Elias, S., and Ciechanover, A. (1983). Components of ubiquitinprotein ligase system. Resolution, affinity purification, and role in protein breakdown. fournal of Biological Chemistry, 258(13):8206-8214.

Hicke, L. (2001). Protein regulation by monoubiquitin . Nat Rev Mol Cell Biol, 2(3):195201.

Higginbotham, H. R. and Gleeson, J. G. (2007). The centrosome in neuronal development. Trends in Neurosciences, 30(6):276-283.

Hikida, T., Jaaro-Peled, H., Seshadri, S., Oishi, K., Hookway, C., Kong, S., Wu, D., Xue, R., Andradé, M., Tankou, S., Mori, S., Gallagher, M., Ishizuka, K., Pletnikov, M., Kida, S., and Sawa, A. (2007). Dominant-negative DISC1 transgenic mice display schizophrenia-associated phenotypes detected by measures translatable to humans. Proceedings of the National Academy of Sciences, 104(36):14501-14506.

Hirota, T., Lee, J. W., St. John, P. C., Sawa, M., Iwaisako, K., Noguchi, T., Pongsawakul, P. Y., Sonntag, T., Welsh, D. K., Brenner, D. A., Doyle, F. J., Schultz, P. G., and Kay, S. A. (2012). Identification of Small Molecule Activators of Cryptochrome. Science, 337(6098):1094-1097.

Hochstrasser, M. (2009). Origin and function of ubiquitin-like proteins. Nature, 458(7237):422-429.

Hoeck, J. D., Jandke, A., Blake, S. M., Nye, E., Spencer-Dene, B., Brandner, S., and Behrens, A. (2010). Fbw7 controls neural stem cell differentiation and progenitor apoptosis via Notch and c-Jun. Nat Neurosci, 13(11):1365-1372.

Hollis II, E. R., Lu, P., Blesch, A., and Tuszynski, M. H. (2009). IGF-I gene delivery promotes corticospinal neuronal survival but not regeneration after adult CNS injury. Experimental Neurology, 215(1):53-59. 
Houlihan, L. M., Harris, S. E., Luciano, M., Gow, A. J., Starr, J. M., Visscher, P. M., and Deary, I. J. (2009). Replication study of candidate genes for cognitive abilities: the Lothian Birth Cohort 1936. Genes, Brain and Behavior, 8(2):238-247.

Hu, G., Zhang, S., Vidal, M., Baer, J. L., Xu, T., and Fearon, E. R. (1997). Mammalian homologs of seven in absentia regulate DCC via the ubiquitin-proteasome pathway. Genes \& Development, 11(20):2701-2714.

Huber, A. B., Kolodkin, A. L., Ginty, D. D., and Cloutier, J.-F. (2003). SIGNALING AT THE GROWTH CONE: Ligand-Receptor Complexes and the Control of Axon Growth and Guidance. Annual Review of Neuroscience, 26(1):509-563.

Hwu, H.-G., Liu, C.-M., Fann, C. S.-J., Ou-Yang, W.-C., and Lee, S. F.-C. (2003). Linkage of schizophrenia with chromosome 1q loci in Taiwanese families. Mol Psychiatry, $8(4): 445-452$.

Ikeda, F. and Dikic, I. (2008). Atypical ubiquitin chains: new molecular signals. EMBO Rep, 9(6):536-542.

Ikeuchi, Y., Stegmüller, J., Netherton, S., Huynh, M. A., Masu, M., Frank, D., Bonni, S., and Bonni, A. (2009). A SnoN-Ccd1 Pathway Promotes Axonal Morphogenesis in the Mammalian Brain. The fournal of Neuroscience, 29(13):4312-4321.

Ishizuka, K., Kamiya, A., Oh, E. C., Kanki, H., Seshadri, S., Robinson, J. F., Murdoch, H., Dunlop, A. J., Kubo, K.-i., Furukori, K., Huang, B., Zeledon, M., Hayashi-Takagi, A., Okano, H., Nakajima, K., Houslay, M. D., Katsanis, N., and Sawa, A. (2011a). DISC1dependent switch from progenitor proliferation to migration in the developing cortex. Nature, 473(7345):92-6.

Ishizuka, K., Kamiya, A., Oh, E. C., Kanki, H., Seshadri, S., Robinson, J. F., Murdoch, H., Dunlop, A. J., Kubo, K.-i., Furukori, K., Huang, B., Zeledon, M., Hayashi-Takagi, A., Okano, H., Nakajima, K., Houslay, M. D., Katsanis, N., and Sawa, A. (2011b). DISC1dependent switch from progenitor proliferation to migration in the developing cortex. Nature, 473(7345):92-6. 
James, R., Adams, R. R., Christie, S., Buchanan, S. R., Porteous, D. J., and Millar, J. K. (2004). Disrupted in Schizophrenia 1 (DISC1) is a multicompartmentalized protein that predominantly localizes to mitochondria. Molecular and cellular neurosciences, 26(1):112-22.

Jan, Y.-N. and Jan, L. Y. (2003). The Control of Dendrite Development. Neuron, 40(2):229242.

Jandke, A., Da Costa, C., Sancho, R., Nye, E., Spencer-Dene, B., and Behrens, A. (2011). The F-box protein Fbw7 is required for cerebellar development. Developmental Biology, 358(1):201-212.

Jiang, H., Guo, W., Liang, X., and Rao, Y. (2005). Both the Establishment and the Maintenance of Neuronal Polarity Require Active Mechanisms: Critical Roles of GSK-3 $\beta$ and Its Upstream Regulators. Cell, 120(1):123-135.

Jin, J., Cardozo, T., Lovering, R. C., Elledge, S. J., Pagano, M., and Harper, J. W. (2004). Systematic analysis and nomenclature of mammalian F-box proteins. Genes \& Development, 18(21):2573-2580.

Kamiya, A., Kubo, K.-i., Tomoda, T., Takaki, M., Youn, R., Ozeki, Y., Sawamura, N., Park, U., Kudo, C., Okawa, M., Ross, C. a., Hatten, M. E., Nakajima, K., and Sawa, A. (2005). A schizophrenia-associated mutation of DISC1 perturbs cerebral cortex development. Nature cell biology, 7(12):1167-78.

Kamiya, A., Tan, P., Kubo, K., Engelhard, C., Ishizuka, K., Kubo, A., Tsukita, S., Pulver, A., Nakajima, K., Cascella, N., Katsanis, N., and Sawa, A. (2008). Recruitment of pcm 1 to the centrosome by the cooperative action of disc1 and bbs4: A candidate for psychiatric illnesses. Archives of General Psychiatry, 65(9):6-996.

Kamiya, A., Tomoda, T., Chang, J., Takaki, M., Zhan, C., Morita, M., Cascio, M. B., Elashvili, S., Koizumi, H., Takanezawa, Y., Dickerson, F., Yolken, R., Arai, H., and Sawa, A. (2006). DISC1-NDEL1/NUDEL protein interaction, an essential component 
for neurite outgrowth, is modulated by genetic variations of DISC1. Human molecular genetics, 15(22):3313-23.

Kannan, M., Lee, S.-J., Schwedhelm-Domeyer, N., Nakazawa, T., and Stegmüller, J. (2012a). p250GAP is a novel player in the Cdh1-APC/Smurf1 pathway of axon growth regulation. PloS one, 7(11):e50735.

Kannan, M., Lee, S.-J., Schwedhelm-Domeyer, N., and Stegmüller, J. (2012b). The E3 ligase Cdh1-anaphase promoting complex operates upstream of the E3 ligase Smurf1 in the control of axon growth. Development, 139(19):3600-3612.

Kara, S., Jissendi-Tchofo, P., and Barkovich, A. J. (2010). Developmental Differences of the Major Forebrain Commissures in Lissencephalies. American fournal of Neuroradiology, 31(9):1602-1607.

Kato, M. and Dobyns, W. B. (2003). Lissencephaly and the molecular basis of neuronal migration. Human Molecular Genetics, 12(suppl 1):R89-R96.

Katz, L. C. and Shatz, C. J. (1996). Synaptic Activity and the Construction of Cortical Circuits. Science, 274(5290):1133-1138.

Kawabe, H., Neeb, A., Dimova, K., Young, S. M., Takeda, M., Katsurabayashi, S., Mitkovski, M., Malakhova, O. a., Zhang, D.-E., Umikawa, M., Kariya, K.-i., Goebbels, S., Nave, K.-A., Rosenmund, C., Jahn, O., Rhee, J., and Brose, N. (2010). Regulation of Rap2A by the ubiquitin ligase Nedd4-1 controls neurite development. Neuron, 65(3):358-72.

Kawaji, K., Umeshima, H., Eiraku, M., Hirano, T., and Kengaku, M. (2004). Dual phases of migration of cerebellar granule cells guided by axonal and dendritic leading processes. Molecular and Cellular Neuroscience, 25(2):228-240.

Kennedy, T. E. (2000). Cellular mechanisms of netrin function: Long-range and shortrange actions. Biochemistry and Cell Biology, 78(5):569-575. 
Kerwin, R. W., Patel, S., Meldrum, B. S., Czudek, C., and Reynolds, G. P. (1988). ASYMMETRICAL LOSS OF GLUTAMATE RECEPTOR SUBTYPE IN LEFT HIPPOCAMPUS IN SCHIZOPHRENIA. The Lancet, 331(8585):583-584.

Kholmanskikh, S. S., Dobrin, J. S., Wynshaw-Boris, A., Letourneau, P. C., and Ross, M. E. (2003). Disregulated RhoGTPases and Actin Cytoskeleton Contribute to the Migration Defect in Lis1-Deficient Neurons. The fournal of Neuroscience, 23(25):8673-8681.

Kholmanskikh, S. S., Koeller, H. B., Wynshaw-Boris, A., Gomez, T., Letourneau, P. C., and Ross, M. E. (2006). Calcium-dependent interaction of Lis1 with IQGAP1 and Cdc42 promotes neuronal motility. Nat Neurosci, 9(1):50-57.

Kim, H. C. and Huibregtse, J. M. (2009). Polyubiquitination by HECT E3s and the Determinants of Chain Type Specificity. Molecular and Cellular Biology, 29(12):3307-3318.

Kim, J., Kim, J., Kim, D., Ryu, G., Bae, S., and Seo, Y. (2004). SCFhFBH1 can act as helicase and E3 ubiquitin ligase. Nucleic Acids Research, 32(8):2287-2297.

Kim, J., Kim, J.-H., Lee, S.-H., Kim, D.-H., Kang, H.-Y., Bae, S.-H., Pan, Z.-Q., and Seo, Y.-S. (2002). The Novel Human DNA Helicase hFBH1 Is an F-box Protein. fournal of Biological Chemistry, 277(27):24530-24537.

Kim, J. Y., Duan, X., Liu, C. Y., Jang, M.-H., Guo, J. U., Pow-anpongkul, N., Kang, E., Song, H., and Ming, G.-1. (2009). DISC1 Regulates New Neuron Development in the Adult Brain via Modulation of AKT-mTOR Signaling through KIAA1212. Neuron, 63(6):761-773.

Kim, T.-H., Lee, H. K., Seo, I. A., Bae, H. R., Suh, D. J., Wu, J., Rao, Y., Hwang, K.G., and Park, H. T. (2005). Netrin induces down-regulation of its receptor, Deleted in Colorectal Cancer, through the ubiquitin-proteasome pathway in the embryonic cortical neuron. Journal of Neurochemistry, 95(1):1-8.

Kitagawa, K., Skowyra, D., Elledge, S. J., Harper, J., and Hieter, P. (1999). SGT1 Encodes an Essential Component of the Yeast Kinetochore Assembly Pathway and a Novel Subunit of the SCF Ubiquitin Ligase Complex. Molecular Cell, 4(1):21-33. 
Komander, D. and Rape, M. (2012). The Ubiquitin Code. Annual Review of Biochemistry, 81(1):203-229.

Komuro, H. and Rakic, P. (1995). Dynamics of granule cell migration: a confocal microscopic study in acute cerebellar slice preparations. The fournal of Neuroscience, 15(2):1110-1120.

Konishi, Y., Stegmüller, J., Matsuda, T., Bonni, S., and Bonni, A. (2004). Cdh1-APC controls axonal growth and patterning in the mammalian brain. Science (New York, N.Y.), 303(5660):1026-30.

Kuchay, S., Duan, S., Schenkein, E., Peschiaroli, A., Saraf, A., Florens, L., Washburn, M. P., and Pagano, M. (2013). FBXL2- and PTPL1-mediated degradation of p110free $\mathrm{p} 85 \beta$ regulatory subunit controls the $\mathrm{PI}(3) \mathrm{K}$ signalling cascade. Nat Cell Biol, 15(5):472-480.

Kullander, K. and Klein, R. (2002). Mechanisms and functions of eph and ephrin signalling. Nat Rev Mol Cell Biol, 3(7):475-486.

Lasorella, A., Stegmuller, J., Guardavaccaro, D., Liu, G., Carro, M. S., Rothschild, G., de la Torre-Ubieta, L., Pagano, M., Bonni, A., and Iavarone, A. (2006). Degradation of Id2 by the anaphase-promoting complex couples cell cycle exit and axonal growth. Nature, 442(7101):471-474.

Lawrie, S. M., Whalley, H., Kestelman, J. N., Abukmeil, S. S., Byrne, M., Hodges, A., Rimmington, J. E., Best, J. J. K., Owens, D. G. C., and Johnstone, E. C. (1999). Magnetic resonance imaging of brain in people at high risk of developing schizophrenia. The Lancet, 353(9146):30-33.

Letourneau, P. C. (1983). Differences in the organization of actin in the growth cones compared with the neurites of cultured neurons from chick embryos. The fournal of Cell Biology, 97(4):963-973.

Lewis, D. A. and Levitt, P. (2002). SCHIZOPHRENIA AS A DISORDER OF NEURODEVELOPMENT. Annual Review of Neuroscience, 25(1):409-432. 
Liang, Y., Yu, W., Li, Y., Yang, Z., Yan, X., Huang, Q., and Zhu, X. (2004). Nudel functions in membrane traffic mainly through association with Lis1 and cytoplasmic dynein. The fournal of Cell Biology, 164(4):557-566.

Liang, Y., Yu, W., Li, Y., Yu, L., Zhang, Q., Wang, F., Yang, Z., Du, J., Huang, Q., Yao, X., and Zhu, X. (2007). Nudel Modulates Kinetochore Association and Function of Cytoplasmic Dynein in M Phase. Molecular Biology of the Cell, 18(7):2656-2666.

Liao, E. H., Hung, W., Abrams, B., and Zhen, M. (2004). An SCF-like ubiquitin ligase complex that controls presynaptic differentiation. Nature, 430(6997):345-350.

Lips, E. S., Cornelisse, L. N., Toonen, R. F., Min, J. L., Hultman, C. M., Holmans, P. a., O’Donovan, M. C., Purcell, S. M., Smit, a. B., Verhage, M., Sullivan, P. F., Visscher, P. M., and Posthuma, D. (2012). Functional gene group analysis identifies synaptic gene groups as risk factor for schizophrenia. Molecular psychiatry, 17(10):996-1006.

Lipska, B. K., Peters, T., Hyde, T. M., Halim, N., Horowitz, C., Mitkus, S., Weickert, C. S., Matsumoto, M., Sawa, A., Straub, R. E., Vakkalanka, R., Herman, M. M., Weinberger, D. R., and Kleinman, J. E. (2006). Expression of DISC1 binding partners is reduced in schizophrenia and associated with DISC1 SNPs. Human Molecular Genetics, 15(8):1245-1258.

Litterman, N., Ikeuchi, Y., Gallardo, G., O’Connell, B. C., Sowa, M. E., Gygi, S. P., Harper, J. W., and Bonni, A. (2011). An OBSL1-Cul7< <up $>$ Fbxw8</sup $>$ Ubiquitin Ligase Signaling Mechanism Regulates Golgi Morphology and Dendrite Patterning. PLoS Biol, 9(5):e1001060.

Liu, J. S. (2011). Molecular genetics of neuronal migration disorders. Current neurology and neuroscience reports, 11(2):171-8.

Löw, K., Culbertson, M., Bradke, F., Tessier-Lavigne, M., and Tuszynski, M. H. (2008). Netrin-1 Is a Novel Myelin-Associated Inhibitor to Axon Growth. The fournal of Neuroscience, 28(5):1099-1108. 
Luskin, M. B. (1993). Restricted proliferation and migration of postnatally generated neurons derived from the forebrain subventricular zone. Neuron, 11(1):173-189.

Lyuksyutova, A. I., Lu, C.-C., Milanesio, N., King, L. A., Guo, N., Wang, Y., Nathans, J., Tessier-Lavigne, M., and Zou, Y. (2003). Anterior-Posterior Guidance of Commissural Axons by Wnt-Frizzled Signaling. Science, 302(5652):1984-1988.

Ma, L., Liu, Y., Ky, B., Shughrue, P. J., Austin, C. P., and Morris, J. A. (2002). Cloning and Characterization of Disc1, the Mouse Ortholog of DISC1 (Disrupted-in-Schizophrenia 1). Genomics, 80(6):662-672.

Ma, L., Tsai, M.-Y., Wang, S., Lu, B., Chen, R., III, J. R. Y., Zhu, X., and Zheng, Y. (2009). Requirement for Nudel and dynein for assembly of the lamin B spindle matrix. Nat Cell Biol, 11(3):247-256.

Macgregor, S., Visscher, P. M., Knott, S. A., Thomson, P., Porteous, D. J., Millar, J. K., Devon, R. S., Blackwood, D., and Muir, W. J. (2004). A genome scan and followup study identify a bipolar disorder susceptibility locus on chromosome 1q42. Mol Psychiatry, 9(12):1083-1090.

Mao, Y., Ge, X., Frank, C. L., Madison, J. M., Koehler, A. N., Doud, M. K., Tassa, C., Berry, E. M., Soda, T., Singh, K. K., Biechele, T., Petryshen, T. L., Moon, R. T., Haggarty, S. J., and Tsai, L.-H. (2009). Disrupted in schizophrenia 1 regulates neuronal progenitor proliferation via modulation of GSK3beta/beta-catenin signaling. Cell, 136(6):101731.

Maria, B. L., Boltshauser, E., Palmer, S. C., and Tran, T. X. (1999). Clinical Features and Revised Diagnostic Criteria in Joubert Syndrome. Fournal of Child Neurology, 14(9):583-590.

Marin, O. and Rubenstein, J. L. R. (2001). A long, remarkable journey: Tangential migration in the telencephalon. Nat Rev Neurosci, 2(11):780-790.

Markus, A., Patel, T. D., and Snider, W. D. (2002a). Neurotrophic factors and axonal growth. Current Opinion in Neurobiology, 12(5):523-531. 
Markus, A., Zhong, J., and Snider, W. D. (2002b). Raf and Akt Mediate Distinct Aspects of Sensory Axon Growth. Neuron, 35(1):65-76.

Marín, O., Yaron, A., Bagri, A., Tessier-Lavigne, M., and Rubenstein, J. L. R. (2001). Sorting of Striatal and Cortical Interneurons Regulated by Semaphorin-Neuropilin Interactions. Science, 293(5531):872-875.

Métin, C., Vallee, R. B., Rakic, P., and Bhide, P. G. (2008). Modes and Mishaps of Neuronal Migration in the Mammalian Brain. The fournal of Neuroscience, 28(46):11746-11752.

Meyer, K. D. and Morris, J. a. (2009). Disc1 regulates granule cell migration in the developing hippocampus. Human molecular genetics, 18(17):3286-97.

Millar, J., Christie, S., Semple, C. A. M., and Porteous, D. J. (2000a). Chromosomal Location and Genomic Structure of the Human Translin-Associated Factor X Gene (TRAX; TSNAX) Revealed by Intergenic Splicing to DISC1, a Gene Disrupted by a Translocation Segregating with Schizophrenia. Genomics, 67(1):69-77.

Millar, J. K., Christie, S., Anderson, S., Lawson, D., Hsiao-Wei Loh, D., Devon, R. S., Arveiler, B., Muir, W. J., Blackwood, D. H., and Porteous, D. J. (2001). Genomic structure and localisation within a linkage hotspot of Disrupted In Schizophrenia 1, a gene disrupted by a translocation segregating with schizophrenia. Molecular psychiatry, 6(2):173-8.

Millar, J. K., Wilson-Annan, J. C., Anderson, S., Christie, S., Taylor, M. S., Semple, C. a., Devon, R. S., St Clair, D. M., Muir, W. J., Blackwood, D. H., and Porteous, D. J. (2000b). Disruption of two novel genes by a translocation co-segregating with schizophrenia. Human molecular genetics, 9(9):1415-23.

Millen, K. J. and Gleeson, J. G. (2008). Cerebellar development and disease. Current Opinion in Neurobiology, 18(1):12-19.

Miyoshi, K., Honda, A., Baba, K., Taniguchi, M., Oono, K., Fujita, T., Kuroda, S., Katayama, T., and Tohyama, M. (2003). Disrupted-In-Schizophrenia 1, a candidate 
gene for schizophrenia, participates in neurite outgrowth. Mol Psychiatry, 8(7):685694.

Moores, C. A., Perderiset, M., Francis, F., Chelly, J., Houdusse, A., and Milligan, R. A. (2004). Mechanism of Microtubule Stabilization by Doublecortin. Molecular Cell, 14(6):833-839.

Mori, D., Yamada, M., Mimori-Kiyosue, Y., Shirai, Y., Suzuki, A., Ohno, S., Saya, H., Wynshaw-Boris, A., and Hirotsune, S. (2009). An essential role of the aPKC-Aurora A-NDEL1 pathway in neurite elongation by modulation of microtubule dynamics. Nature cell biology, 11(9):1057-68.

Mori, D., Yano, Y., Toyo-oka, K., Yoshida, N., Yamada, M., Muramatsu, M., Zhang, D., Saya, H., Toyoshima, Y. Y., Kinoshita, K., Wynshaw-Boris, A., and Hirotsune, S. (2007). NDEL1 Phosphorylation by Aurora-A Kinase Is Essential for Centrosomal Maturation, Separation, and TACC3 Recruitment. Molecular and Cellular Biology, 27(1):352367.

Morris, J. A., Kandpal, G., Ma, L., and Austin, C. P. (2003). DISC1 (Disrupted-InSchizophrenia 1) is a centrosome-associated protein that interacts with MAP1A, MIPT3, ATF4/5 and NUDEL: regulation and loss of interaction with mutation. Human Molecular Genetics, 12(13):1591-1608.

Namba, T., Ming, G.-1., Song, H., Waga, C., Enomoto, A., Kaibuchi, K., Kohsaka, S., and Uchino, S. (2011). NMDA receptor regulates migration of newly generated neurons in the adult hippocampus via Disrupted-In-Schizophrenia 1 (DISC1). Fournal of Neurochemistry, 118(1):34-44.

Nato, A., Kong, X., Cui, A., Brzustowicz, L., and Matise, T. (2006). Genomic characterization of schizophrenia candidate gene regions.

Nelson, R. F., Glenn, K. A., Zhang, Y., Wen, H., Knutson, T., Gouvion, C. M., Robinson, B. K., Zhou, Z., Yang, B., Smith, R. J. H., and Paulson, H. L. (2007). Selective Cochlear 
Degeneration in Mice Lacking the F-Box Protein, Fbx2, a Glycoprotein-Specific Ubiquitin Ligase Subunit. The fournal of Neuroscience, 27(19):5163-5171.

Nguyen, M. D., Shu, T., Sanada, K., Larivière, R. C., Tseng, H.-C., Park, S. K., Julien, J.-P., and Tsai, L.-H. (2004). A NUDEL-dependent mechanism of neurofilament assembly regulates the integrity of CNS neurons. Nature cell biology, 6(7):595-608.

Niclou, S. P., Franssen, E. H. P., Ehlert, E. M. E., Taniguchi, M., and Verhaagen, J. (2003). Meningeal cell-derived semaphorin 3A inhibits neurite outgrowth. Molecular and Cellular Neuroscience, 24(4):902-912.

Niwa, M., Kamiya, A., Murai, R., Kubo, K.-i., Gruber, A. J., Tomita, K., Lu, L., Tomisato, S., Jaaro-Peled, H., Seshadri, S., Hiyama, H., Huang, B., Kohda, K., Noda, Y., O’Donnell, P., Nakajima, K., Sawa, A., and Nabeshima, T. (2010). Knockdown of DISC1 by in utero gene transfer disturbs postnatal dopaminergic maturation in the frontal cortex and leads to adult behavioral deficits. Neuron, 65(4):480-9.

Nobes, C. D. and Hall, A. (1995). Rho, Rac, and Cdc42 GTPases regulate the assembly of multimolecular focal complexes associated with actin stress fibers, lamellipodia, and filopodia. Cell, 81(1):53-62.

Nóbrega-Pereira, S., Kessaris, N., Du, T., Kimura, S., Anderson, S. A., and Marín, O. (2008). Postmitotic Nkx2-1 Controls the Migration of Telencephalic Interneurons by Direct Repression of Guidance Receptors. Neuron, 59(5):733-745.

Ogawa, F., Kasai, M., and Akiyama, T. (2005). A functional link between Disrupted-InSchizophrenia 1 and the eukaryotic translation initiation factor 3. Biochemical and Biophysical Research Communications, 338(2):771-776.

Oinuma, I., Katoh, H., and Negishi, M. (2007). R-Ras Controls Axon Specification Upstream of Glycogen Synthase Kinase- $3 \beta$ through Integrin-linked Kinase. Journal of Biological Chemistry, 282(1):303-318. 
Okabe, H., Lee, S.-H., Phuchareon, J., Albertson, D. G., McCormick, F., and Tetsu, O. (2006). A Critical Role for FBXW8 and MAPK in Cyclin D1 Degradation and Cancer Cell Proliferation. PLoS ONE, 1(1):e128.

Okuda, A., Kishi, T., Okochi, T., Ikeda, M., Kitajima, T., Tsunoka, T., Okumukura, T., Fukuo, Y., Kinoshita, Y., Kawashima, K., Yamanouchi, Y., Inada, T., Ozaki, N., and Iwata, N. (2010). Translin-Associated Factor X Gene (TSNAX) may be Associated with Female major Depressive Disorder in the Japanese Population. NeuroMolecular Medicine, 12(1):78-85.

Oprea, G. E., Kröber, S., McWhorter, M. L., Rossoll, W., Müller, S., Krawczak, M., Bassell, G. J., Beattie, C. E., and Wirth, B. (2008). Plastin 3 Is a Protective Modifier of Autosomal Recessive Spinal Muscular Atrophy. Science, 320(5875):524-527.

Orlicky, S., Tang, X., Neduva, V., Elowe, N., Brown, E. D., Sicheri, F., and Tyers, M. (2010). An allosteric inhibitor of substrate recognition by the SCFCdc4 ubiquitin ligase. Nat Biotech, 28(7):733-737.

Ozeki, Y., Tomoda, T., Kleiderlein, J., Kamiya, A., Bord, L., Fujii, K., Okawa, M., Yamada, N., Hatten, M. E., Snyder, S. H., Ross, C. A., and Sawa, A. (2003). Disruptedin-Schizophrenia-1 (DISC-1): Mutant truncation prevents binding to NudE-like (NUDEL) and inhibits neurite outgrowth. Proceedings of the National Academy of Sciences, 100(1):289-294.

Pak, C. W., Flynn, K. C., and Bamburg, J. R. (2008). Actin-binding proteins take the reins in growth cones. Nat Rev Neurosci, 9(2):136-147.

Parisi, M. A. and Dobyns, W. B. (2003). Human malformations of the midbrain and hindbrain: review and proposed classification scheme. Molecular Genetics and Metabolism, 80(1-2):36-53.

Pasterkamp, R. J. and Kolodkin, A. L. (2003). Semaphorin junction: making tracks toward neural connectivity. Current Opinion in Neurobiology, 13(1):79-89. 
Paylor, R., Hirotsune, S., Gambello, M. J., Yuva-Paylor, L., Crawley, J. N., and WynshawBoris, A. (1999). Impaired Learning and Motor Behavior in Heterozygous Pafah1b1 (Lis1) Mutant Mice. Learning \& Memory, 6(5):521-537.

Pletnikov, M. V., Ayhan, Y., Xu, Y., Nikolskaia, O., Ovanesov, M., Huang, H., Mori, S., Moran, T. H., and Ross, C. A. (2008). Enlargement of the lateral ventricles in mutant DISC1 transgenic mice. Mol Psychiatry, 13(2):115.

Polleux, F., Whitford, K. L., Dijkhuizen, P. A., Vitalis, T., and Ghosh, A. (2002). Control of cortical interneuron migration by neurotrophins and PI3-kinase signaling. Development, 129(13):3147-3160.

Ponyeam, W. and Hagen, T. (2012). Characterization of the Cullin7 E3 ubiquitin ligase - Heterodimerization of cullin substrate receptors as a novel mechanism to regulate cullin E3 ligase activity. Cellular Signalling, 24(1):290-295.

Popovich, P. and McTigue, D. (2009). Damage control in the nervous system: beware the immune system in spinal cord injury. Nat Med, 15(7):736-737.

Pozas, E. and Ibáñez, C. F. (2005). GDNF and GFR $\alpha 1$ Promote Differentiation and Tangential Migration of Cortical GABAergic Neurons. Neuron, 45(5):701-713.

Purro, S. A., Ciani, L., Hoyos-Flight, M., Stamatakou, E., Siomou, E., and Salinas, P. C. (2008). Wnt Regulates Axon Behavior through Changes in Microtubule Growth Directionality: A New Role for Adenomatous Polyposis Coli. The fournal of Neuroscience, 28(34):8644-8654.

Rakic, P. (1971). Neuron-glia relationship during granule cell migration in developing cerebellar cortex. A Golgi and electonmicroscopic study in Macacus rhesus. The fournal of Comparative Neurology, 141(3):283-312.

Rakic, P. (1972). Mode of cell migration to the superficial layers of fetal monkey neocortex. The fournal of Comparative Neurology, 145(1):61-83. 
Rakic, P. (1978). Neuronal migration and contact guidance in the primate telencephalon. Postgraduate Medical fournal, 54(Supplement 1):25-40.

Ramon y Cajal, S. (1995). Histology of the Nervous System of Man and Vertebrates. Oxford University Press, USA, 1 edition.

Rauch, A., Thiel, C. T., Schindler, D., Wick, U., Crow, Y. J., Ekici, A. B., van Essen, A. J., Goecke, T. O., Al-Gazali, L., Chrzanowska, K. H., Zweier, C., Brunner, H. G., Becker, K., Curry, C. J., Dallapiccola, B., Devriendt, K., Dörfler, A., Kinning, E., Megarbane, A., Meinecke, P., Semple, R. K., Spranger, S., Toutain, A., Trembath, R. C., Voss, E., Wilson, L., Hennekam, R., de Zegher, F., Dörr, H.-G., and Reis, A. (2008). Mutations in the Pericentrin (PCNT) Gene Cause Primordial Dwarfism. Science, 319(5864):816-819.

Ravid, T. and Hochstrasser, M. (2007). Autoregulation of an E2 enzyme by ubiquitinchain assembly on its catalytic residue. Nat Cell Biol, 9(4):422-427.

Ravid, T. and Hochstrasser, M. (2008). Diversity of degradation signals in the ubiquitinproteasome system. Nat Rev Mol Cell Biol, 9(9):679-689.

Reiner, O., Albrecht, U., Gordon, M., Chianese, A., Buchberg, A. M., Thomas, C., Wang, C., Gal-gerber, O., and Eichele, G. (1995). Lissencephaly Gene ( LISI ) Expression in Neuronal Migration in the CNS Suggests a Role. The fournal of Neuroscience, 15(May):3730-3738.

Reiner, O., Carrozzo, R., Shen, Y., Wehnert, M., Faustinella, F., Dobyns, W. B., Caskey, C. T., and Ledbetter, D. H. (1993). Isolation of a Miller-Dicker lissencephaly gene containing G protein [beta]-subunit-like repeats. Nature, 364(6439):717-721.

Ridley, A. J., Paterson, H. F., Johnston, C. L., Diekmann, D., and Hall, A. (1992). The small GTP-binding protein rac regulates growth factor-induced membrane ruffling. Cell, 70(3):401-410.

Rivas, R. J., Hatten, M. E., and York, N. (1995). Motility and cytoskeletal organization of migrating cerebellar granule neurons. The fournal of Neuroscience, 15(2):981-989. 
Ross, M. E., Allen, K. M., Srivastava, A. K., Featherstone, T., Gleeson, J. G., Hirsch, B., Harding, B. N., Andermann, E., Abdullah, R., Berg, M., Czapansky-Bielman, D., Flanders, D. J., Guerrini, R., Motté, J., Mira, A. P., Scheffer, I., Berkovic, S., Scaravilli, F., King, R. A., Ledbetter, D. H., Schlessinger, D., Dobyns, W. B., and Walsh, C. A. (1997). Linkage and Physical Mapping of X-Linked Lissencephaly/SBH (XLIS): A Gene Causing Neuronal Migration Defects in Human Brain. Human Molecular Genetics, 6(4):555-562.

Rossoll, W., Jablonka, S., Andreassi, C., Kröning, A.-K., Karle, K., Monani, U. R., and Sendtner, M. (2003). Smn, the spinal muscular atrophy-determining gene product, modulates axon growth and localization of $\beta$-actin mRNA in growth cones of motoneurons. The fournal of Cell Biology, 163(4):801-812.

Rotin, D. and Kumar, S. (2009). Physiological functions of the HECT family of ubiquitin ligases. Nat Rev Mol Cell Biol, 10(6):398-409.

Ryder, E. F. and Cepko, C. L. (1994). Migration patterns of clonally related granule cells and their progenitors in the developing chick cerebellum. Neuron, 12(5):1011-1029.

Sabry, J. H., O’Connor, T. P., Evans, L., Toroian-Raymond, A., Kirschner, M., and Bentley, D. (1991). Microtubule behavior during guidance of pioneer neuron growth cones in situ. The fournal of Cell Biology, 115(2):381-395.

Saiga, T., Fukuda, T., Matsumoto, M., Tada, H., Okano, H. J., Okano, H., and Nakayama, K. I. (2009). Fbxo45 forms a novel ubiquitin ligase complex and is required for neuronal development. Molecular and cellular biology, 29(13):3529-43.

Sakaguchi, T., Okada, M., Kitamura, T., and Kawasaki, K. (1993). Reduced diameter and conduction velocity of myelinated fibers in the sciatic nerve of a neurofilamentdeficient mutant quail. Neuroscience Letters, 153(1):65-68.

Sakamoto, K. M., Kim, K. B., Kumagai, A., Mercurio, F., Crews, C. M., and Deshaies, R. J. (2001). Protacs: Chimeric molecules that target proteins to the Skp1-Cullin-F box 
complex for ubiquitination and degradation. Proceedings of the National Academy of Sciences, 98(15):8554-8559.

Sakamoto, K. M., Kim, K. B., Verma, R., Ransick, A., Stein, B., Crews, C. M., and Deshaies, R. J. (2003). Development of Protacs to Target Cancer-promoting Proteins for Ubiquitination and Degradation. Molecular \& Cellular Proteomics, 2(12):1350-1358.

Sanders, A. R., Duan, J., Levinson, D. F., Shi, J., He, D., Hou, C., Burrell, G. J., Rice, J. P., Nertney, D. a., Olincy, A., Rozic, P., Vinogradov, S., Buccola, N. G., Mowry, B. J., Freedman, R., Amin, F., Black, D. W., Silverman, J. M., Byerley, W. F., Crowe, R. R., Cloninger, C. R., Martinez, M., and Gejman, P. V. (2008). No significant association of 14 candidate genes with schizophrenia in a large European ancestry sample: implications for psychiatric genetics. The American journal of psychiatry, 165(4):497-506.

Sarikas, A., Xu, X., Field, L. J., and Pan, Z.-Q. (2008). The cullin7 E3 ubiquitin ligase: a novel player in growth control. Cell cycle Georgetown Tex, 7(20):3154-3161.

Sasaki, S., Mori, D., Toyo-oka, K., Chen, A., Garrett-Beal, L., Muramatsu, M., Miyagawa, S., Hiraiwa, N., Yoshiki, A., Wynshaw-Boris, A., and Hirotsune, S. (2005). Complete Loss of Ndel1 Results in Neuronal Migration Defects and Early Embryonic Lethality. Molecular and Cellular Biology, 25(17):7812-7827.

Sasaki, S., Shionoya, A., Ishida, M., Gambello, M. J., Yingling, J., Wynshaw-Boris, A., and Hirotsune, S. (2000). A LIS1/NUDEL/Cytoplasmic Dynein Heavy Chain Complex in the Developing and Adult Nervous System. Neuron, 28(3):681-696.

Sawa, A. and Roberts, R. C. (2006). DISC1 Immunoreactivity at the Light and Ultrastructural Level in the Human Neocortex. The fournal of Comparative Neurology, 450(April 2005):436-450.

Scheffner, M., Nuber, U., and Huibregtse, J. M. (1995). Protein ubiquitination involving an E1-E2-E3 enzyme ubiquitin thioester cascade. Nature, 373(6509):81-83. 
Schmid, R. S., Jo, R., Shelton, S., Kreidberg, J. A., and Anton, E. S. (2005). Reelin, Integrin and Dab1 Interactions during Embryonic Cerebral Cortical Development. Cerebral Cortex, 15(10):1632-1636.

Schurov, I. L., Handford, E. J., Brandon, N. J., and Whiting, P. J. (2004). Expression of disrupted in schizophrenia 1 (DISC1) protein in the adult and developing mouse brain indicates its role in neurodevelopment. Molecular psychiatry, 9(12):1100-10.

Schwamborn, J. C., Muller, M., Becker, A. H. M., and Puschel, A. W. (2007). Ubiquitination of the GTPase Rap1B by the ubiquitin ligase Smurf2 is required for the establishment of neuronal polarity. EMBO f, 26(5):1410-1422.

Segarra, J., Balenci, L., Drenth, T., Maina, F., and Lamballe, F. (2006). Combined Signaling through ERK, PI3K/AKT, and RAC1/p38 Is Required for Met-triggered Cortical Neuron Migration. Journal of Biological Chemistry, 281(8):4771-4778.

Shen, Y., Li, N., Wu, S., Zhou, Y., Shan, Y., Zhang, Q., Ding, C., Yuan, Q., Zhao, F., Zeng, R., and Zhu, X. (2008). Nudel binds Cdc42GAP to modulate Cdc42 activity at the leading edge of migrating cells. Developmental cell, 14(3):342-53.

Shim, S. Y., Samuels, B. A., Wang, J., Neumayer, G., Belzil, C., Ayala, R., Shi, Y., Shi, Y., Tsai, L.-H., and Nguyen, M. D. (2008). Ndel1 Controls the Dynein-mediated Transport of Vimentin during Neurite Outgrowth. Fournal of Biological Chemistry, 283(18):12232-12240.

Shimizu, S., Matsuzaki, S., Hattori, T., Kumamoto, N., Miyoshi, K., Katayama, T., and Tohyama, M. (2008). DISC1-kendrin interaction is involved in centrosomal microtubule network formation. Biochemical and Biophysical Research Communications, 377(4):1051-1056.

Shinoda, T., Taya, S., Tsuboi, D., Hikita, T., Matsuzawa, R., Kuroda, S., Iwamatsu, A., and Kaibuchi, K. (2007). DISC1 Regulates Neurotrophin-Induced Axon Elongation via Interaction with Grb2. The fournal of Neuroscience, 27(1):4-14. 
Shmueli, A., Segal, M., Sapir, T., Tsutsumi, R., Noritake, J., Bar, A., Sapoznik, S., Fukata, Y., Orr, I., Fukata, M., and Reiner, O. (2010). Ndel1 palmitoylation: a new mean to regulate cytoplasmic dynein activity. EMBO f, 29(1):107-119.

Shoukimas, G. M. and Hinds, J. W. (1978). The development of the cerebral cortex in the embryonic mouse: An electron microscopic serial section analysis. The fournal of Comparative Neurology, 179(4):795-830.

Shu, T., Ayala, R., Nguyen, M.-D., Xie, Z., Gleeson, J. G., and Tsai, L.-H. (2004). Ndel1 operates in a common pathway with LIS1 and cytoplasmic dynein to regulate cortical neuronal positioning. Neuron, 44(2):263-277.

Silver, J. and Miller, J. H. (2004). Regeneration beyond the glial scar. Nat Rev Neurosci, 5(2):146-156.

Simó, S., Jossin, Y., and Cooper, J. A. (2010). Cullin 5 Regulates Cortical Layering by Modulating the Speed and Duration of Dab1-Dependent Neuronal Migration. The fournal of Neuroscience, 30(16):5668-5676.

Singh, K. K., Ge, X., Mao, Y., Drane, L., Meletis, K., Samuels, B. a., and Tsai, L.-H. (2010). Dixdc1 is a critical regulator of DISC1 and embryonic cortical development. Neuron, 67(1):33-48.

Skaar, J. R., D’Angiolella, V., Pagan, J. K., and Pagano, M. (2009). SnapShot: F Box Proteins II. Cell, 137(7):1358.e1-1358.e2.

Skaar, J. R., Pagan, J. K., and Pagano, M. (2013). Mechanisms and function of substrate recruitment by F-box proteins. Nature reviews. Molecular cell biology, 14(6):369-81.

Skowyra, D., Craig, K. L., Tyers, M., Elledge, S. J., and Harper, J. W. (1997). F-Box Proteins Are Receptors that Recruit Phosphorylated Substrates to the SCF UbiquitinLigase Complex. Cell, 91(2):209-219.

Solecki, D. J., Model, L., Gaetz, J., Kapoor, T. M., and Hatten, M. E. (2004). Par6[alpha] signaling controls glial-guided neuronal migration. Nat Neurosci, 7(11):1195-1203. 
Solecki, D. J., Trivedi, N., Govek, E.-E., Kerekes, R. a., Gleason, S. S., and Hatten, M. E. (2009). Myosin II motors and F-actin dynamics drive the coordinated movement of the centrosome and soma during CNS glial-guided neuronal migration. Neuron, 63(1):6380 .

Song, H.-j. and Poo, M.-m. (2001). The cell biology of neuronal navigation. Nat Cell Biol, 3(3):E81-E88.

Sossey-Alaoui, K., Hartung, a. J., Guerrini, R., Manchester, D. K., Posar, a., Puche-Mira, a., Andermann, E., Dobyns, W. B., and Srivastava, a. K. (1998). Human doublecortin (DCX) and the homologous gene in mouse encode a putative Ca2+-dependent signaling protein which is mutated in human X-linked neuronal migration defects. Human molecular genetics, 7(8):1327-32.

Sperbera, B. R., Leight, S., Goedert, M., and Lee, V.-Y. (1995). Glycogen synthase kinase$3 \beta$ phosphorylates tau protein at multiple sites in intact cells. Neuroscience Letters, 197(2):149-153.

Splinter, D., Tanenbaum, M. E., Lindqvist, A., Jaarsma, D., Flotho, A., Yu, K. L., Grigoriev, I., Engelsma, D., Haasdijk, E. D., Keijzer, N., Demmers, J., Fornerod, M., Melchior, F., Hoogenraad, C. C., Medema, R. H., and Akhmanova, A. (2010). Bicaudal D2, Dynein, and Kinesin-1 Associate with Nuclear Pore Complexes and Regulate Centrosome and Nuclear Positioning during Mitotic Entry. PLoS Biol, 8(4):e1000350.

St Clair, D., Blackwood, D., Muir, W., Walker, M., Carothers, A., Spowart, G., Gosden, C., and Evans, H. J. (1990). Association within a family of a balanced autosomal translocation with major mental illness. The Lancet, 336(8706):13-16.

Stegmüller, J., Konishi, Y., Huynh, M. A., Yuan, Z., Dibacco, S., and Bonni, A. (2006). Cellintrinsic regulation of axonal morphogenesis by the Cdh1-APC target SnoN. Neuron, 50(3):389-400.

Stiess, M., Maghelli, N., Kapitein, L. C., Gomis-Rüth, S., Wilsch-Bräuninger, M., Hoogenraad, C. C., Tolić-N $\varnothing$ rrelykke, I. M., and Bradke, F. (2010). Axon extension oc- 
curs independently of centrosomal microtubule nucleation. Science (New York, N.Y.), 327(5966):704-7.

Suter, D. M. and Forscher, P. (2000). Substrate-cytoskeletal coupling as a mechanism for the regulation of growth cone motility and guidance. fournal of Neurobiology, 44(2):97-113.

Takei, Y., Kondo, S., Harada, A., Inomata, S., Noda, T., and Hirokawa, N. (1997). Delayed Development of Nervous System in Mice Homozygous for Disrupted Microtubuleassociated Protein 1B (MAP1B) Gene. The fournal of Cell Biology, 137(7):1615-1626.

Takei, Y., Teng, J., Harada, A., and Hirokawa, N. (2000). Defects in Axonal Elongation and Neuronal Migration in Mice with Disrupted tau and map1b Genes. The fournal of Cell Biology, 150(5):989-1000.

Tamamaki, N., Fujimori, K. E., and Takauji, R. (1997). Origin and Route of Tangentially Migrating Neurons in the Developing Neocortical Intermediate Zone. The fournal of Neuroscience, 17(21):8313-8323.

Tanaka, E., Ho, T., and Kirschner, M. W. (1995). The role of microtubule dynamics in growth cone motility and axonal growth. The fournal of Cell Biology, 128(1):139-155.

Tanaka, E. and Kirschner, M. W. (1995). The role of microtubules in growth cone turning at substrate boundaries. The fournal of Cell Biology, 128(1):127-137.

Tanaka, E. and Sabry, J. (1995). Making the connection: Cytoskeletal rearrangements during growth cone guidance. Cell, 83(2):171-176.

Tanaka, T., Serneo, F. F., Higgins, C., Gambello, M. J., Wynshaw-Boris, A., and Gleeson, J. G. (2004). Lis1 and doublecortin function with dynein to mediate coupling of the nucleus to the centrosome in neuronal migration. The fournal of cell biology, 165(5):709-21.

Taya, S., Shinoda, T., Tsuboi, D., Asaki, J., Nagai, K., Hikita, T., Kuroda, S., Kuroda, K., Shimizu, M., Hirotsune, S., Iwamatsu, A., and Kaibuchi, K. (2007). DISC1 Regulates 
the Transport of the NUDEL/LIS1/14-3-3 $\epsilon$ Complex through Kinesin-1. The fournal of Neuroscience, 27(1):15-26.

Taylor, M. S., Devon, R. S., Millar, J., and Porteous, D. J. (2003). Evolutionary constraints on the Disrupted in Schizophrenia locus. Genomics, 81(1):67-77.

Tessier-Lavigne, M. and Goodman, C. S. (1996). The Molecular Biology of Axon Guidance. Science, 274(5290):1123-1133.

Tomita, K., Kubo, K.-i., Ishii, K., and Nakajima, K. (2011). Disrupted-in-Schizophrenia-1 (Disc1) is necessary for migration of the pyramidal neurons during mouse hippocampal development. Human molecular genetics, 20(14):2834-45.

Torisawa, T., Nakayama, A., Furuta, K., Yamada, M., Hirotsune, S., and Toyoshima, Y. Y. (2011). Functional Dissection of LIS1 and NDEL1 Towards Understanding the Molecular Mechanisms of Cytoplasmic Dynein Regulation. fournal of Biological Chemistry, 286(3):1959-1965.

Toth, C., Shim, S. Y., Wang, J., Jiang, Y., Neumayer, G., Belzil, C., Liu, W.-Q., Martinez, J., Zochodne, D., and Nguyen, M. D. (2008). Ndel1 Promotes Axon Regeneration via Intermediate Filaments. PLoS ONE, 3(4):e2014.

Toyo-Oka, K., Sasaki, S., Yano, Y., Mori, D., Kobayashi, T., Toyoshima, Y. Y., Tokuoka, S. M., Ishii, S., Shimizu, T., Muramatsu, M., Hiraiwa, N., Yoshiki, A., Wynshaw-Boris, A., and Hirotsune, S. (2005). Recruitment of katanin p60 by phosphorylated NDEL1, an LIS1 interacting protein, is essential for mitotic cell division and neuronal migration. Human molecular genetics, 14(21):3113-28.

Tsai, J.-W., Bremner, K. H., and Vallee, R. B. (2007). Dual subcellular roles for LIS1 and dynein in radial neuronal migration in live brain tissue. Nature neuroscience, 10(8):970-9.

Tsai, L.-H. and Gleeson, J. G. (2005). Nucleokinesis in Neuronal Migration. Neuron, 46(3):383-388. 
Tuszynski, M.H., Lu, P. (2008). Axonal plasticity and regeneration in the injured spinal cord. In Kordower, J.H., Tuszynski, M., editor, CNS regeneration: basic science and clinical advances, pages 319-337. Academic Press, London.

Umeshima, H., Hirano, T., and Kengaku, M. (2007). Microtubule-based nuclear movement occurs independently of centrosome positioning in migrating neurons. Proceedings of the National Academy of Sciences of the United States of America, 104(41):16182-7.

Vadhvani, M., Schwedhelm-Domeyer, N., Mukherjee, C., and Stegmüller, J. (2013). The Centrosomal E3 Ubiquitin Ligase FBXO31-SCF Regulates Neuronal Morphogenesis and Migration. PLoS ONE, 8(2):e57530.

Vallee, R. B., Seale, G. E., and Tsai, J.-W. (2009). Emerging roles for myosin II and cytoplasmic dynein in migrating neurons and growth cones. Trends in cell biology, 19(7):347-55.

Venables, P. H. (1992). Hippocampal Function and Schizophrenia. Experimental Psychological Evidencea. Annals of the New York Academy of Sciences, 658(1):111-127.

Wang, H.-R., Zhang, Y., Ozdamar, B., Ogunjimi, A. A., Alexandrova, E., Thomsen, G. H., and Wrana, J. L. (2003). Regulation of Cell Polarity and Protrusion Formation by Targeting RhoA for Degradation. Science, 302(5651):1775-1779.

Wang, X., Herr, R. A., Chua, W.-J., Lybarger, L., Wiertz, E. J. H. J., and Hansen, T. H. (2007). Ubiquitination of serine, threonine, or lysine residues on the cytoplasmic tail can induce ERAD of MHC-I by viral E3 ligase mK3. The fournal of Cell Biology, 177(4):613-624.

Watanabe, N., Madaule, P., Reid, T., Ishizaki, T., Watanabe, G., Kakizuka, A., Saito, Y., Nakao, K., Jockusch, B. M., and Narumiya, S. (1997). p140mDia, a mammalian homolog of Drosophila diaphanous,is a target protein for Rho small GTPase and is a ligand for profilin. EMBO F, 16(11):3044-3056.

Watanabe, T., von der Kammer, H., Wang, X., Shintani, Y., and Horiguchi, T. (2013). Neuronal Expression of F-Box and Leucine-Rich-Repeat Protein 2 Decreases over 
Braak Stages in the Brains of Alzheimer's Disease Patients. Neurodegenerative Diseases, 11(1):1-12.

Wei, W., Jin, J., Schlisio, S., Harper, J. W., and Kaelin Jr., W. G. (2005). The v-Jun point mutation allows c-Jun to escape GSK3-dependent recognition and destruction by the Fbw7 ubiquitin ligase. Cancer Cell, 8(1):25-33.

Welcker, M. and Clurman, B. E. (2008). FBW7 ubiquitin ligase: a tumour suppressor at the crossroads of cell division, growth and differentiation. Nat Rev Cancer, 8(2):83-93.

Westbrook, T. F., Hu, G., Ang, X. L., Mulligan, P., Pavlova, N. N., Liang, A., Leng, Y., Maehr, R., Shi, Y., Harper, J. W., and Elledge, S. J. (2008). SCFbeta-TRCP controls oncogenic transformation and neural differentiation through REST degradation. $\mathrm{Na}$ ture, 452(7185):370-4.

Wichterle, H., García-Verdugo, J. M., and Alvarez-Buylla, A. (1997). Direct Evidence for Homotypic, Glia-Independent Neuronal Migration. Neuron, 18(5):779-791.

Wichterle, H., Garcia-Verdugo, J. M., Herrera, D. G., and Alvarez-Buylla, A. (1999). Young neurons from medial ganglionic eminence disperse in adult and embryonic brain. Nat Neurosci, 2(5):461-466.

Widenfalk, J., Lundströmer, K., Jubran, M., Brené, S., and Olson, L. (2001). Neurotrophic Factors and Receptors in the Immature and Adult Spinal Cord after Mechanical Injury or Kainic Acid. The fournal of Neuroscience, 21(10):3457-3475.

Wingate, R. J. and Hatten, M. E. (1999). The role of the rhombic lip in avian cerebellum development. Development, 126(20):4395-4404.

Winzeler, A. M., Mandemakers, W. J., Sun, M. Z., Stafford, M., Phillips, C. T., and Barres, B. A. (2011). The Lipid Sulfatide Is a Novel Myelin-Associated Inhibitor of CNS Axon Outgrowth. The fournal of Neuroscience, 31(17):6481-6492.

Witte, H., Neukirchen, D., and Bradke, F. (2008). Microtubule stabilization specifies initial neuronal polarization. The fournal of Cell Biology, 180(3):619-632. 
Wong, E. and Cuervo, A. M. (2010). Integration of Clearance Mechanisms: The Proteasome and Autophagy. Cold Spring Harbor Perspectives in Biology, 2(12).

Wu, C.-H., Fallini, C., Ticozzi, N., Keagle, P. J., Sapp, P. C., Piotrowska, K., Lowe, P., Koppers, M., McKenna-Yasek, D., Baron, D. M., Kost, J. E., Gonzalez-Perez, P., Fox, A. D., Adams, J., Taroni, F., Tiloca, C., Leclerc, A. L., Chafe, S. C., Mangroo, D., Moore, M. J., Zitzewitz, J. A., Xu, Z.-S., van den Berg, L. H., Glass, J. D., Siciliano, G., Cirulli, E. T., Goldstein, D. B., Salachas, F., Meininger, V., Rossoll, W., Ratti, A., Gellera, C., Bosco, D. A., Bassell, G. J., Silani, V., Drory, V. E., Brown Jr, R. H., and Landers, J. E. (2012a). Mutations in the profilin 1 gene cause familial amyotrophic lateral sclerosis. Nature, 488(7412):499-503.

Wu, G., Xu, G., Schulman, B. A., Jeffrey, P. D., Harper, J., and Pavletich, N. P. (2003). Structure of a $\beta$-TrCP1-Skp1- $\beta$-Catenin Complex: Destruction Motif Binding and Lysine Specificity of the SCF $\beta$-TrCP1 Ubiquitin Ligase. Molecular Cell, 11(6):1445-1456.

Wu, L., Grigoryan, A., Li, Y., Hao, B., Pagano, M., and Cardozo, T. (2012b). Specific Small Molecule Inhibitors of Skp2-Mediated p27 Degradation. Chemistry \& Biology, 19(12):1515-1524.

Wu, X., Johansen, J., and Helin, K. (2013). Fbxl10/Kdm2b Recruits Polycomb Repressive Complex 1 to CpG Islands and Regulates H2A Ubiquitylation. Molecular Cell, 49(6):1134-1146.

Xiao, J., Pradhan, A., and Liu, Y. (2006). Functional role of JNK in neuritogenesis of PC12-N1 cells. Neuroscience Letters, 392(3):231-234.

Xie, F. and Zheng, B. (2008). White matter inhibitors in CNS axon regeneration failure. Experimental Neurology, 209(2):302-312.

Xu, X., Sarikas, A., Dias-Santagata, D. C., Dolios, G., Lafontant, P. J., Tsai, S.-C., Zhu, W., Nakajima, H., Nakajima, H. O., Field, L. J., Wang, R., and Pan, Z.-Q. (2008). The CUL7 E3 Ubiquitin Ligase Targets Insulin Receptor Substrate 1 for Ubiquitin-Dependent Degradation. Molecular Cell, 30(4):403-414. 
Yamada, M., Toba, S., Yoshida, Y., Haratani, K., Mori, D., Yano, Y., Mimori-Kiyosue, Y., Nakamura, T., Itoh, K., Fushiki, S., Setou, M., Wynshaw-Boris, A., Torisawa, T., Toyoshima, Y. Y., and Hirotsune, S. (2008). LIS1 and NDEL1 coordinate the plus-enddirected transport of cytoplasmic dynein. EMBO f, 27(19):2471-2483.

Yau, H.-J., Wang, H.-F., Lai, C., and Liu, F.-C. (2003). Neural Development of the Neuregulin Receptor ErbB4 in the Cerebral Cortex and the Hippocampus: Preferential Expression by Interneurons Tangentially Migrating from the Ganglionic Eminences. Cerebral Cortex, 13(3):252-264.

Yoshimura, T., Kawano, Y., Arimura, N., Kawabata, S., Kikuchi, A., and Kaibuchi, K. (2005). GSK-3 $\beta$ Regulates Phosphorylation of CRMP-2 and Neuronal Polarity. Cell, 120(1):137-149.

Young-Pearse, T. L., Bai, J., Chang, R., Zheng, J. B., LoTurco, J. J., and Selkoe, D. J. (2007). A Critical Function for $\beta$-Amyloid Precursor Protein in Neuronal Migration Revealed by In Utero RNA Interference. The fournal of Neuroscience, 27(52):14459-14469.

Young-Pearse, T. L., Suth, S., Luth, E. S., Sawa, A., and Selkoe, D. J. (2010). Biochemical and functional interaction of disrupted-in-schizophrenia 1 and amyloid precursor protein regulates neuronal migration during mammalian cortical development. The fournal of neuroscience : the official journal of the Society for Neuroscience, 30(31):1043140.

Zhang, Q., Wang, F., Cao, J., Shen, Y., Huang, Q., Bao, L., and Zhu, X. (2009a). Nudel Promotes Axonal Lysosome Clearance and Endo-lysosome Formation via DyneinMediated Transport. Traffic, 10(9):1337-1349.

Zhang, X., Lei, K., Yuan, X., Wu, X., Zhuang, Y., Xu, T., Xu, R., and Han, M. (2009b). SUN1/2 and Syne/Nesprin-1/2 Complexes Connect Centrosome to the Nucleus during Neurogenesis and Neuronal Migration in Mice. Neuron, 64(2):173-187.

Zhao, T., De Graaff, E., Breedveld, G. J., Loda, A., Severijnen, L.-A., Wouters, C. H., Verheijen, F. W., Dekker, M. C. J., Montagna, P., Willemsen, R., Oostra, B. A., and 
Bonifati, V. (2011). Loss of Nuclear Activity of the FBXO7 Protein in Patients with Parkinsonian-Pyramidal Syndrome (PARK15). PLoS ONE, 6(2):e16983.

Zheng, N., Schulman, B. A., Song, L., Miller, J. J., Jeffrey, P. D., Wang, P., Chu, C., Koepp, D. M., Elledge, S. J., Pagano, M., Conaway, R. C., Conaway, J. W., Harper, J. W., and Pavletich, N. P. (2002). Structure of the Cul1-Rbx1-Skp1-F boxSkp2 SCF ubiquitin ligase complex. Nature, 416(6882):703-709.

Zhou, F.-Q. and Snider, W. D. (2006). Intracellular control of developmental and regenerative axon growth. Philosophical Transactions of the Royal Society B: Biological Sciences, 361(1473):1575-1592.

Zhu, J., Burakov, A., Rodionov, V., and Mogilner, A. (2010). Finding the Cell Center by a Balance of Dynein and Myosin Pulling and Microtubule Pushing: A Computational Study. Molecular Biology of the Cell, 21(24):4418-4427.

Zmuda, J. F. and Rivas, R. J. (1998). The Golgi apparatus and the centrosome are localized to the sites of newly emerging axons in cerebellar granule neurons in vitro. Cell Motility and the Cytoskeleton, 41(1):18-38.

Żyłkiewicz, E., Kijańska, M., Choi, W.-C., Derewenda, U., Derewenda, Z. S., and Stukenberg, P. T. (2011). The N-terminal coiled-coil of Ndel1 is a regulated scaffold that recruits LIS1 to dynein. The fournal of Cell Biology, 192(3):433-445. 


\section{Anna Holubowska}

curriculum vitae

Personal information

date of birth 11th August 1984

place of birth Wałcz, Poland

\section{Education}

2009-present PhD Molecular and Cellular Neurobiology, Max Planck Institute of Experimental Medicine, Göttingen, Germany.

2006-2008 MSc Biotechnology, Adam Mickiewicz University, Poznan, Poland, very good.

2003-2006 BSc Biotechnology, Adam Mickiewicz University, Poznan, Poland, very good.

\section{Experience}

2009-present PhD thesis, Planck Institute of Experimental Medicine, Laboratory of Cellular and Molecular Neurobiology, Göttingen, Germany.

"Characterization of the CNS-specific F-box protein FBXO41 in cerebellar development", Dr. J. Stegmüller

2008-2009 Research project, Georg-August-Universität, Institute of Human Genetics, Göttingen, Germany.

"Non-coding RNAs in pluripotency and development", Dr.med. A. Zovoilis

2006-2008 MSc thesis, Polish Academy of Sciences, Institute of Bioorganic Chemistry, Poznań, Poland.

"Structure and function of non-coding RNAs", Prof. J. Barciszewski

2007 Research projects, Georg-August-Universität, Department of Plant Biochemistry, Göttingen, Germany.

"Expression of truncated variants or overexpression of the PIPK3-gene affects root hair development in Arabidopsis thaliana", "Heterologous expression, purification and crystallization of modified variants of Arabidopsis phosphatidylinositol-4phosphate 5-kinase 3", Dr. I. Heilmann 


\section{Publications}

Holubowska, A., Mukherjee, C., Schwedhelm-Domeyer, N., Matz, A., Kannan, M., Vadhvani, M., and Stegmüller, J. Loss of the central nervous system-specific F-box protein FBXO41 models early-onset ataxia in mice. In preparation.

Holubowska, A., Mukherjee, C., Vadhvani, M., and Stegmueller, J. (2013). Genetic manupulation of cerebellar granule neurons in vitro and in vivo to study neuronal morphology and migration. JoVE, In press.

Stenzel, I., Ischebeck, T., König, S., Hołubowska, A., Sporysz, M., Hause, B., and Heilmann, I. (2008). The Type B Phosphatidylinositol-4-Phosphate 5-Kinase 3 Is Essential for Root Hair Formation in Arabidopsis thaliana. The Plant Cell Online, 20(1):124-141.

Zovoilis, A., Pantazi, A., Smorag, L., Opitz, L., Riester, G. S., Wolf, M., Zechner, U., Holubowska, A., Stewart, C. L., and Engel, W. (2010). Embryonic stem cell-related miRNAs are involved in differentiation of pluripotent cells originating from the germ line. Molecular Human Reproduction, 16(11):793803.

\section{Scholarships}

○ "Socrates-Erasmus" Scholarship, European Commission 